\title{
Submillimeter $\mathrm{H}_{2} \mathrm{O}$ and $\mathrm{H}_{2} \mathrm{O}^{+}$emission in lensed ultra- and hyper-luminous infrared galaxies at $z \sim 2-4^{\star, \star \star}$
}

\author{
C. Yang (杨辰涛) $)^{1,2,3,4,5}$, A. Omont ${ }^{4,5}$, A. Beelen ${ }^{2}$, E. González-Alfonso ${ }^{6}$, R. Neri ${ }^{7}$, Y. Gao (高显) $)^{1}$, P. van der Werf ${ }^{8}$, \\ A. Weiß ${ }^{9}$, R. Gavazzi ${ }^{4,5}$, N. Falstad ${ }^{10}$, A. J. Baker ${ }^{11}$, R. S. Bussmann ${ }^{12}$, A. Cooray ${ }^{13}$, P. Cox ${ }^{14}$, H. Dannerbauer ${ }^{15}$, \\ S. Dye ${ }^{16}$, M. Guélin ${ }^{7}$, R. Ivison ${ }^{17,18}$, M. Krips ${ }^{7}$, M. Lehnert ${ }^{4,5}$, M. J. Michałowski ${ }^{17}$, \\ D. A. Riechers ${ }^{12}$, M. Spaans ${ }^{19}$, and E. Valiante ${ }^{20}$ \\ (Affiliations can be found after the references)
}

Received 19 January 2016 / Accepted 20 July 2016

\begin{abstract}
We report rest-frame submillimeter $\mathrm{H}_{2} \mathrm{O}$ emission line observations of 11 ultra- or hyper-luminous infrared galaxies (ULIRGs or HyLIRGs) at $z \sim 2-4$ selected among the brightest lensed galaxies discovered in the Herschel-Astrophysical Terahertz Large Area Survey (H-ATLAS). Using the IRAM NOrthern Extended Millimeter Array (NOEMA), we have detected 14 new $\mathrm{H}_{2} \mathrm{O}$ emission lines. These include five $3_{21}-3_{12}$ ortho- $\mathrm{H}_{2} \mathrm{O}$ lines $\left(E_{\text {up }} / k=305 \mathrm{~K}\right)$ and nine $J=2$ para- $\mathrm{H}_{2} \mathrm{O}$ lines, either $2_{02}-1_{11}\left(E_{\mathrm{up}} / k=101 \mathrm{~K}\right)$ or $2_{11}-2_{02}\left(E_{\mathrm{up}} / k=137 \mathrm{~K}\right)$. The apparent luminosities of the $\mathrm{H}_{2} \mathrm{O}$ emission lines are $\mu L_{\mathrm{H}_{2} \mathrm{O}} \sim 6-21 \times 10^{8} L_{\odot}(3<\mu<15$, where $\mu$ is the lens magnification factor), with velocity-integrated line fluxes ranging from 4-15 Jy km s${ }^{-1}$. We have also observed CO emission lines using EMIR on the IRAM $30 \mathrm{~m}$ telescope in seven sources (most of those have not yet had their $\mathrm{CO}$ emission lines observed). The velocity widths for $\mathrm{CO}$ and $\mathrm{H}_{2} \mathrm{O}$ lines are found to be similar, generally within $1 \sigma$ errors in the same source. With almost comparable integrated flux densities to those of the high- $J \mathrm{CO}$ line (ratios range from 0.4 to 1.1 ), $\mathrm{H}_{2} \mathrm{O}$ is found to be among the strongest molecular emitters in high-redshift Hy/ULIRGs. We also confirm our previously found correlation between luminosity of $\mathrm{H}_{2} \mathrm{O}\left(L_{\mathrm{H}_{2} \mathrm{O}}\right)$ and infrared $\left(L_{\mathrm{IR}}\right)$ that $L_{\mathrm{H}_{2} \mathrm{O}} \sim L_{\mathrm{IR}}{ }^{1.1-1.2}$, with our new detections. This correlation could be explained by a dominant role of far-infrared pumping in the $\mathrm{H}_{2} \mathrm{O}$ excitation. Modelling reveals that the far-infrared radiation fields have warm dust temperature $T_{\text {warm }} \sim 45-75 \mathrm{~K}, \mathrm{H}_{2} \mathrm{O}$ column density per unit velocity interval $N_{\mathrm{H}_{2} \mathrm{O}} / \Delta V \gtrsim 0.3 \times 10^{15} \mathrm{~cm}^{-2} \mathrm{~km}^{-1} \mathrm{~s}$ and $100 \mu \mathrm{m}$ continuum opacity $\tau_{100}>1$ (optically thick), indicating that $\mathrm{H}_{2} \mathrm{O}$ is likely to trace highly obscured warm dense gas. However, further observations of $J \geq 4 \mathrm{H}_{2} \mathrm{O}$ lines are needed to better constrain the continuum optical depth and other physical conditions of the molecular gas and dust. We have also detected $\mathrm{H}_{2} \mathrm{O}^{+}$emission in three sources. A tight correlation between $L_{\mathrm{H}_{2} \mathrm{O}}$ and $L_{\mathrm{H}_{2} \mathrm{O}^{+}}$has been found in galaxies from low to high redshift. The velocity-integrated flux density ratio between $\mathrm{H}_{2} \mathrm{O}^{+}$and $\mathrm{H}_{2} \mathrm{O}$ suggests that cosmic rays generated by strong star formation are possibly driving the $\mathrm{H}_{2} \mathrm{O}^{+}$formation.
\end{abstract}

Key words. galaxies: high-redshift - galaxies: ISM - infrared: galaxies - submillimeter: galaxies - radio lines: ISM - ISM: molecules

\section{Introduction}

After molecular hydrogen $\left(\mathrm{H}_{2}\right)$ and carbon monoxide $(\mathrm{CO})$, the water molecule $\left(\mathrm{H}_{2} \mathrm{O}\right)$ can be one of the most abundant molecules in the interstellar medium (ISM) in galaxies. It provides some important diagnostic tools for various physical and chemical processes in the ISM (e.g. van Dishoeck et al. 2013, and references therein). Prior to the Herschel Space Observatory (Pilbratt et al. 2010), in extragalactic sources, nonmaser $\mathrm{H}_{2} \mathrm{O}$ rotational transitions were only detected by the Infrared Space Observatory (ISO, Kessler et al. 1996) in the form of far-infrared absorption lines (González-Alfonso et al. 2004, 2008). Observations of local infrared bright galaxies by Herschel have revealed a rich spectrum of submillimeter (submm) $\mathrm{H}_{2} \mathrm{O}$ emission lines (submm $\mathrm{H}_{2} \mathrm{O}$ refers to rest-frame submillimeter $\mathrm{H}_{2} \mathrm{O}$ emission throughout this paper if not otherwise specified). Many of these lines are emitted from high-excitation rotational levels with upper-level energies up to $E_{\text {up }} / k=642 \mathrm{~K}$

\footnotetext{
* Herschel is an ESA space observatory with science instruments provided by European-led Principal Investigator consortia and with important participation from NASA.

$\star \star$ The reduced spectra as FITS files are only available at the CDS via anonymous ftp to cdsarc.u-strasbg. fr (130.79.128.5) or via http://cdsarc.u-strasbg.fr/viz-bin/qcat?J/A+A/595/A80
}

(e.g. van der Werf et al. 2010; González-Alfonso et al. 2010, 2012, 2013; Rangwala et al. 2011; Kamenetzky et al. 2012; Spinoglio et al. 2012; Meijerink et al. 2013; Pellegrini et al. 2013; Pereira-Santaella et al. 2013). Excitation analysis of these lines has revealed that they are probably excited through absorption of far-infrared photons from thermal dust emission in warm dense regions of the ISM (e.g. González-Alfonso et al. 2010). Therefore, unlike the canonical $\mathrm{CO}$ lines that trace collisional excitation of the molecular gas, these $\mathrm{H}_{2} \mathrm{O}$ lines represent a powerful diagnostic of the far-infrared radiation field.

Using the Herschel archive data, Yang et al. (2013, hereafter Y13) have undertaken a first systematic study of submm $\mathrm{H}_{2} \mathrm{O}$ emission in local infrared galaxies. $\mathrm{H}_{2} \mathrm{O}$ was found to be the strongest molecular emitter after $\mathrm{CO}$ within the submm band in those infrared-bright galaxies, even with higher flux density than that of $\mathrm{CO}$ in some local ULIRGs (velocity-integrated flux density of $\mathrm{H}_{2} \mathrm{O}\left(3_{21}-3_{12}\right)$ is larger than that of $\mathrm{CO}(5-4)$ in four galaxies out of 45 in the Y13 sample). The luminosities of the submm $\mathrm{H}_{2} \mathrm{O}$ lines $\left(L_{\mathrm{H}_{2} \mathrm{O}}\right)$ are near-linearly correlated with total infrared luminosity $\left(L_{\mathrm{IR}}\right.$, integrated over $\left.8-1000 \mu \mathrm{m}\right)$ over three orders of magnitude. The correlation is revealed to be a straightforward result of far-infrared pumping: $\mathrm{H}_{2} \mathrm{O}$ molecules are excited to higher energy levels through absorbing far-infrared photons, then the upper level molecules cascade toward the lines we 
observed in an almost constant fraction (Fig. 1). Although the galaxies dominated by active galactic nuclei (AGN) have somewhat lower ratios of $L_{\mathrm{H}_{2} \mathrm{O}} / L_{\mathrm{IR}}$, there does not appear to be a link between the presence of an AGN and the submm $\mathrm{H}_{2} \mathrm{O}$ emission (Y13). The $\mathrm{H}_{2} \mathrm{O}$ emission is likely to trace the far-infrared radiation field generated in star-forming nuclear regions in galaxies, explaining its tight correlation with far-infrared luminosity.

Besides detections of the $\mathrm{H}_{2} \mathrm{O}$ lines in local galaxies from space telescopes, redshifted submm $\mathrm{H}_{2} \mathrm{O}$ lines in high-redshift lensed Ultra- and Hyper-Luminous InfraRed Galaxies (ULIRGs, $10^{13} L_{\odot}>L_{\mathrm{IR}} \geq 10^{12} L_{\odot} ;$ HyLIRGs, $\left.L_{\mathrm{IR}} \geq 10^{13} L_{\odot}\right)$ can also be detected by ground-based telescopes in atmospheric windows with high transmission. Strong gravitational lensing boosts the flux and allows one to detect the $\mathrm{H}_{2} \mathrm{O}$ emission lines easily. Since our first detection of submm $\mathrm{H}_{2} \mathrm{O}$ in a lensed Herschel source at $z=2.3$ (Omont et al. 2011) using the IRAM NOrthern Extended Millimeter Array (NOEMA), several individual detections at high-redshift have also been reported (Lis et al. 2011; van der Werf et al. 2011; Bradford et al. 2011; Combes et al. 2012; Lupu et al. 2012; Bothwell et al. 2013; Omont et al. 2013; Vieira et al. 2013; Weiß et al. 2013; Rawle et al. 2014). These numerous and easy detections of $\mathrm{H}_{2} \mathrm{O}$ in high-redshift lensed ULIRGs show that its lines are the strongest submm molecular lines after $\mathrm{CO}$ and may be an important tool for studying these galaxies.

We have carried out a series of studies focussing on submm $\mathrm{H}_{2} \mathrm{O}$ emission in high-redshift lensed galaxies since our first detection. Through the detection of $J=2 \mathrm{H}_{2} \mathrm{O}$ lines in seven high-redshift lensed Hy/ULIRGs reported by Omont et al. (2013, hereafter O13), a slightly super-linear correlation between $L_{\mathrm{H}_{2} \mathrm{O}}$ and $L_{\mathrm{IR}}\left(L_{\mathrm{H}_{2} \mathrm{O}} \propto L_{\mathrm{IR}}{ }^{1.2}\right)$ from local ULIRGs and high-redshift lensed Hy/ULIRGs has been found. This result may imply again that far-infrared pumping is important for $\mathrm{H}_{2} \mathrm{O}$ excitation in high-redshift extreme starbursts. The average ratios of $L_{\mathrm{H}_{2} \mathrm{O}}$ to $L_{\mathrm{IR}}$ for the $J=2 \mathrm{H}_{2} \mathrm{O}$ lines in the high-redshift sources tend to be $1.8 \pm 0.9$ times higher than those seen locally (Y13). This shows that the same physics with infrared pumping should dominate $\mathrm{H}_{2} \mathrm{O}$ excitation in ULIRGs at low and high redshift, with some specificity at high-redshift probably linked to the higher luminosities.

Modelling provides additional information about the $\mathrm{H}_{2} \mathrm{O}$ excitation. For example, through LVG modelling, Riechers et al. (2013) argue that the excitation of the submm $\mathrm{H}_{2} \mathrm{O}$ emission in the $z \sim 6.3$ submm galaxy is far-infrared pumping dominated. Modelling of the local Herschel galaxies of Y13 has been carried out by González-Alfonso et al. (2014, hereafter G14). They confirm that far-infrared pumping is the dominant mechanism responsible for the submm $\mathrm{H}_{2} \mathrm{O}$ emission (except for the groundstate emission transitions, such as para- $\mathrm{H}_{2} \mathrm{O}$ transition $1_{11}-0_{00}$ ) in the extragalactic sources. Moreover, collisional excitation of the low-lying $(J \leq 2) \mathrm{H}_{2} \mathrm{O}$ lines could also enhance the radiative pumping of the $(J \geq 3)$ high-lying lines. The ratio between lowlying and high-lying $\mathrm{H}_{2} \mathrm{O}$ lines is sensitive to the dust temperature $\left(T_{\mathrm{d}}\right)$ and $\mathrm{H}_{2} \mathrm{O}$ column density $\left(N_{\mathrm{H}_{2} \mathrm{O}}\right)$. From modelling the average of local star-forming- and mild-AGN-dominated galaxies, $\mathrm{G} 14$ show that the submm $\mathrm{H}_{2} \mathrm{O}$ emission comes from regions with $N_{\mathrm{H}_{2} \mathrm{O}} \sim(0.5-2) \times 10^{17} \mathrm{~cm}^{-2}$ and a $100 \mu \mathrm{m}$ continuum opacity of $\tau_{100} \sim 0.05-0.2$, where $\mathrm{H}_{2} \mathrm{O}$ is mainly excited by warm dust with a temperature range of $45-75 \mathrm{~K}$. $\mathrm{H}_{2} \mathrm{O}$ lines thus provide key information about the properties of the dense cores of ULIRGs, that is, their $\mathrm{H}_{2} \mathrm{O}$ content, the infrared radiation field and the corresponding temperature of dust that is warmer than the core outer layers and dominates the far-infrared emission.
Observations of the submm $\mathrm{H}_{2} \mathrm{O}$ emission, together with appropriate modelling and analysis, therefore allows us to study the properties of the far-infrared radiation sources in great detail. So far, the excitation analysis combining both low- and high-lying $\mathrm{H}_{2} \mathrm{O}$ emission has only been done in a few case studies. Using $\mathrm{H}_{2} \mathrm{O}$ excitation modelling considering both collision and far-infrared pumping, González-Alfonso et al. (2010) and van der Werf et al. (2011) estimate the sizes of the far-infrared radiation fields in Mrk 231 and APM 08279+5255 (APM 08279 hereafter), which are not resolved by the observations directly, and suggest their AGN dominance based on their total enclosed energies. This again demonstrates that submm $\mathrm{H}_{2} \mathrm{O}$ emission is a powerful diagnostic tool which can even transcend the angular resolution of the telescopes.

The detection of submm $\mathrm{H}_{2} \mathrm{O}$ emission in the HerschelATLAS $^{1} \quad$ (Eales et al. 2010, H-ATLAS hereafter) sources through gravitational lensing allows us to characterise the far-infrared radiation field generated by intense star-forming activity, and possibly AGN, and learn the physical conditions in the warm dense gas phase in extreme starbursts in the early Universe. Unlike standard dense gas tracers such as HCN, which is weaker at high-redshift compared to that of local ULIRGs (Gao et al. 2007), submm $\mathrm{H}_{2} \mathrm{O}$ lines are strong and even comparable to high- $J$ CO lines in some galaxies (Y13; O13). Therefore, $\mathrm{H}_{2} \mathrm{O}$ is an efficient tracer of the warm dense gas phase that makes up a major fraction of the total molecular gas mass in high-redshift Hy/ULIRGs (Casey et al. 2014). The successful detections of submm $\mathrm{H}_{2} \mathrm{O}$ lines in both local (Y13) and the high-redshift universe (O13) show the great potential of a systematic study of $\mathrm{H}_{2} \mathrm{O}$ emission in a large sample of infrared galaxies over a wide range in redshift (from local up to $z \sim 4$ ) and luminosity $\left(L_{\mathrm{IR}} \sim 10^{10}-10^{13} L_{\odot}\right)$. However, our previous high-redshift sample was limited to seven sources and to one $J=2$ para- $\mathrm{H}_{2} \mathrm{O}$ line $\left(E_{\text {up }} / k=100-127 \mathrm{~K}\right)$ per source $(\mathrm{O} 13)$. In order to further constrain the conditions of $\mathrm{H}_{2} \mathrm{O}$ excitation, to confirm the dominant role of far-infrared pumping and to learn the physical conditions of the warm dense gas phase in high-redshift starbursts, it is essential to extend the studies to higher excitation lines. We thus present and discuss here the results of such new observations of a strong $J=3$ ortho- $\mathrm{H}_{2} \mathrm{O}$ line with $E_{\text {up }} / k=304 \mathrm{~K}$ in six strongly lensed $H$-ATLAS galaxies at $z \sim 2.8-3.6$, where a second lower-excitation $J=2$ para- $\mathrm{H}_{2} \mathrm{O}$ line was also observed (Fig. 1 for the transitions and the corresponding $E_{\text {up }}$ ).

We describe our sample, observation and data reduction in Section 2. The observed properties of the high-redshift submm $\mathrm{H}_{2} \mathrm{O}$ emission are presented in Sect. 3. Discussions of the lensing properties, $L_{\mathrm{H}_{2} \mathrm{O}}-L_{\mathrm{IR}}$ correlation, $\mathrm{H}_{2} \mathrm{O}$ excitation, comparison between $\mathrm{H}_{2} \mathrm{O}$ and $\mathrm{CO}$, AGN contamination will be given in Sect. 4. Section 5 describes the detection of $\mathrm{H}_{2} \mathrm{O}^{+}$lines. We summarise our results in Sect. 6. A flat $\Lambda \mathrm{CDM}$ cosmology with $H_{0}=71 \mathrm{~km} \mathrm{~s}^{-1} \mathrm{Mpc}^{-1}, \Omega_{\mathrm{M}}=0.27, \Omega_{\Lambda}=0.73$ (Spergel et al. 2003) is adopted throughout this paper.

\section{Sample and observation}

Our sample consists of eleven extremely bright high-redshift sources with $F_{500 \mu \mathrm{m}}>200 \mathrm{mJy}$ discovered by the $H$-ATLAS survey (Eales et al. 2010). Together with the seven similar

\footnotetext{
1 The Herschel-ATLAS is a project with Herschel, which is an ESA space observatory with science instruments provided by European-led Principal Investigator consortia and with important participation from NASA. The $H$-ATLAS website is http://www.h-atlas.org
} 


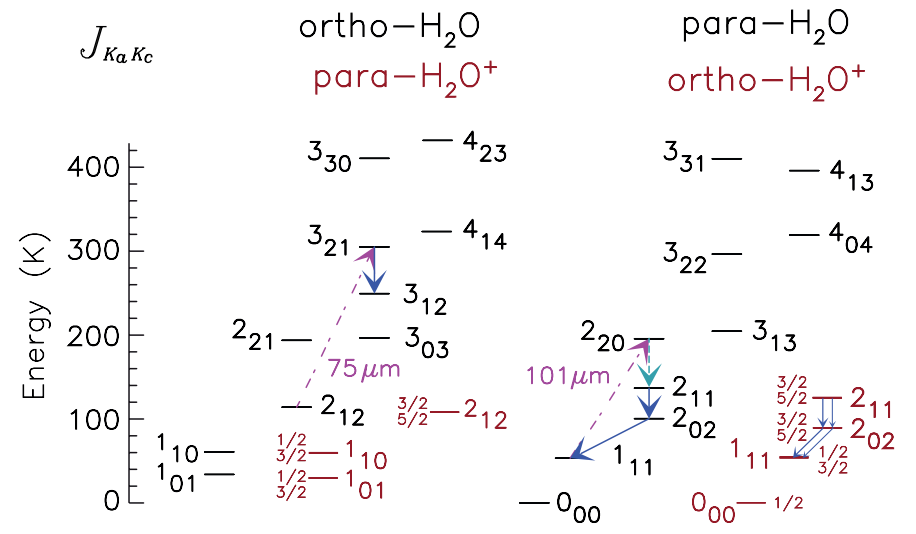

Fig. 1. Energy level diagrams of $\mathrm{H}_{2} \mathrm{O}$ and $\mathrm{H}_{2} \mathrm{O}^{+}$shown in black and red, respectively. Dark blue arrows are the submm $\mathrm{H}_{2} \mathrm{O}$ transitions we have observed in this work. Pink dashed lines show the far-infrared pumping path of the $\mathrm{H}_{2} \mathrm{O}$ excitation in the model we use, with the wavelength of the photon labeled. The light blue dashed arrow is the transition from para- $\mathrm{H}_{2} \mathrm{O}$ energy level $2_{20}$ to $2_{11}$ along the cascade path from $2_{20}$ to $1_{11}$. Rotational energy levels of $\mathrm{H}_{2} \mathrm{O}$ and $\mathrm{H}_{2} \mathrm{O}^{+}$, as well as fine structure component levels of $\mathrm{H}_{2} \mathrm{O}^{+}$are also shown in the figure.

sources reported in our previous $\mathrm{H}_{2} \mathrm{O}$ study (O13), they include all the brightest high-redshift $H$-ATLAS sources $\left(F_{500 \mu \mathrm{m}}>170 \mathrm{mJy}\right)$, but two, imaged at $880 \mu \mathrm{m}$ with SMA by Bussmann et al. (2013, hereafter B13). In agreement with the selection according to the methods of Negrello et al. (2010), the detailed lensing modelling performed by B13 has shown that all of them are strongly lensed, but one, G09v1.124 (Ivison et al. 2013, see below). The sample of our present study is thus well representative of the brightest high-redshift submillimeter sources with $F_{500 \mu \mathrm{m}}>200 \mathrm{mJy}$ (with apparent total infrared luminosity $\sim 5-15 \times 10^{13} L_{\odot}$ and $z \sim 1.5-4.2$ ) found by $H$-ATLAS in its equatorial ("GAMA") and north-galactic-pole ("NGP") fields, in $\sim 300 \mathrm{deg}^{2}$ with a density $\sim 0.05 \mathrm{deg}^{-2}$. In our previous project $(\mathrm{O} 13)$, we observed $\mathrm{H}_{2} \mathrm{O}$ in seven strongly lensed high-redshift $H$-ATLAS galaxies from the B13 sample. In this work, in order to observe the high-excitation ortho$\mathrm{H}_{2} \mathrm{O}\left(3_{21}-3_{12}\right)$ line with rest frequency of $1162.912 \mathrm{GHz}$ with the IRAM/NOEMA, we selected the brightest sources at $500 \mu \mathrm{m}$ with $z \gtrsim 2.8$ so that the redshifted lines could be observed in a reasonably good atmospheric window at $v_{\text {obs }} \lesssim 300 \mathrm{GHz}$. Eight sources with such redshift were selected from the B13 $H$-ATLAS sample.

B13 provide lensing models, magnification factors $(\mu)$ and inferred intrinsic properties of these galaxies and list their $\mathrm{CO}$ redshifts which come from Harris et al. (2012); Harris et al. (in prep.); Lupu et al. (in prep.); Krips et al. (in prep.) and Riechers et al. (in prep.).

In our final selection of the sample to be studied in the $\mathrm{H}_{2} \mathrm{O}\left(3_{21}-3_{12}\right)$ line, we then removed two sources, SDP 81 and G12v2.30, that were previously observed in $\mathrm{H}_{2} \mathrm{O}(\mathrm{O} 13$; and also ALMA Partnership, Vlahakis et al. 2015, for SDP 81), because the $J=2 \mathrm{H}_{2} \mathrm{O}$ emission is too weak and/or the interferometry could resolve out some flux considering the lensing image. The observed high-redshift sample thus consists of two GAMA-field sources: G09v1.97 and G12v2.43, and four sources in the $H$-ATLAS NGP field: NCv1.143, NAv1.195, NAv1.177 and NBv1.78 (Tables 1 and 2). Among the six remaining sources at redshift between 2.8 and 3.6, only one, NBv1.78, has been observed previously in a low-excitation line, para- $\mathrm{H}_{2} \mathrm{O}\left(2_{02}-1_{11}\right)$ (O13). Therefore, we have observed both para- $\mathrm{H}_{2} \mathrm{O}$ line $2_{02}-1_{11}$ or $2_{11}-2_{02}$ and ortho- $\mathrm{H}_{2} \mathrm{O}\left(3_{21}-3_{12}\right)$ in the other five sources, in order to compare their velocity-integrated flux densities.

In addition, we also observed five sources mostly at lower redshifts in para- $\mathrm{H}_{2} \mathrm{O}$ lines $2_{02}-1_{11}$ or $2_{11}-2_{02}$ (Tables 1 and 2) to complete the sample of our $\mathrm{H}_{2} \mathrm{O}$ low-excitation study. They are three strongly lensed sources, G09v1.40, NAv1.56 and SDP11, a hyper-luminous cluster source G09v1.124 (Ivison et al. 2013), and a $z \sim 3.7$ source, NCv1.268 for which we did not propose a $J=3 \mathrm{H}_{2} \mathrm{O}$ observation, considering its large linewidth which could bring difficulties in line detection.

As our primary goal is to obtain a detection of the submm $\mathrm{H}_{2} \mathrm{O}$ lines, we carried out the observations in the compact, D configuration of NOEMA. The baselines extended from 24 to $176 \mathrm{~m}$, resulting in a synthesised beam with modest/low resolution of $\sim 1.0^{\prime \prime} \times 0.9^{\prime \prime}$ to $\sim 5.6^{\prime \prime} \times 3.3^{\prime \prime}$ as shown in Table 1 . The $\mathrm{H}_{2} \mathrm{O}$ observations were conducted from January 2012 to December 2013 in good atmospheric conditions (seeing of $0.3^{\prime \prime}-1.5^{\prime \prime}$ ) stability and reasonable transparency (PWV $\leq 1 \mathrm{~mm}$ ). The total on source time was $\sim 1.5-8 \mathrm{~h}$ per source. $2 \mathrm{~mm}, 1.3 \mathrm{~mm}$ and $0.8 \mathrm{~mm}$ bands covering 129-174, 201-267 and 277-371 GHz, respectively, were used. All the central observation frequencies were chosen based on previous redshifts given by B13 according to the previous $\mathrm{CO}$ detections (Table 2). In all cases but one, the frequencies of our detections of $\mathrm{H}_{2} \mathrm{O}$ lines are consistent with these $\mathrm{CO}$ redshifts. The only exception is G09v1.40 where our $\mathrm{H}_{2} \mathrm{O}$ redshift disagrees with the redshift of $z=2.0894 \pm 0.0009$ given by Lupu et al. (in prep.), which is quoted by B13. We find $z=2.0925 \pm 0.0001$ in agreement with previous $\mathrm{CO}(3-2)$ observations (Riechers et al., in prep.). We used the WideX correlator which provided a contiguous frequency coverage of $3.6 \mathrm{GHz}$ in dual polarisation with a fixed channel spacing of $1.95 \mathrm{MHz}$.

The phase and bandpass were calibrated by measuring standard calibrators that are regularly monitored at the IRAM/NOEMA, including 3C 279, 3C 273, MWC349 and $0923+392$. The accuracy of the flux calibration is estimated to range from $\sim 10 \%$ in the $2 \mathrm{~mm}$ band to $\sim 20 \%$ in the $0.8 \mathrm{~mm}$ band. Calibration, imaging, cleaning and spectra extraction were performed within the GILDAS ${ }^{2}$ packages CLIC and MAPPING.

To compare the $\mathrm{H}_{2} \mathrm{O}$ emission with the typical molecular gas tracer, $\mathrm{CO}$, we also observed the sources for $\mathrm{CO}$ lines using the EMIR receiver at the IRAM $30 \mathrm{~m}$ telescope. The CO data will be part of a systematic study of molecular gas excitation in $H$-ATLAS lensed Hy/ULIRGs, and a full description of the data and the scientific results will be given in a following paper (Yang et al., in prep.). The global $\mathrm{CO}$ emission properties of the sources are listed in Table 3 where we list the CO fluxes and linewidths. A brief comparison of the emission between $\mathrm{H}_{2} \mathrm{O}$ and $\mathrm{CO}$ lines will be given in Sect. 4.3.

\section{Results}

A detailed discussion of the observation results for each source is given in Appendix A, including the strength of the $\mathrm{H}_{2} \mathrm{O}$ emission, the image extension of $\mathrm{H}_{2} \mathrm{O}$ lines and the continuum (Fig. A.1), the $\mathrm{H}_{2} \mathrm{O}$ spectra and linewidths (Fig. 2) and their comparison with CO (Table 3 ). We give a synthesis of these results in this section.

2 See http://www.iram. fr/IRAMFR/GILDAS for more information about the GILDAS softwares. 
Table 1. Observation log.

\begin{tabular}{|c|c|c|c|c|c|c|c|c|c|}
\hline IAU $n$ & S & $\begin{array}{l}\text { RA } \\
(\mathrm{J} 2000)\end{array}$ & $\begin{array}{l}\text { ec } \\
2000)\end{array}$ & $\begin{array}{l}\mathrm{RA}_{\mathrm{pk}} \\
(\mathrm{J} 2000)\end{array}$ & $\begin{array}{l}\operatorname{Dec}_{\mathrm{pk}} \\
(\mathrm{J} 2000)\end{array}$ & $\mathrm{H}_{2} \mathrm{O}$ line & $\begin{array}{l}v_{\text {obs }} \\
(\mathrm{GHz})\end{array}$ & $\begin{array}{l}\text { Beam } \\
\left({ }^{\prime \prime}\right)\end{array}$ & $\begin{array}{l}t_{\text {on }} \\
\text { (h) }\end{array}$ \\
\hline \multirow[t]{2}{*}{$H$-ATLAS J083051.0+013224 } & & 02 & $+01: 32: 2$ & 7 & 39 & & & & 3.5 \\
\hline & & & & & & & & & 3.1 \\
\hline \multirow[t]{2}{*}{$H$-ATLAS J113526.3-014605 } & $\mathrm{C}$ & 36 & -( & & & & & & 6.9 \\
\hline & & & & & & & & & 1.5 \\
\hline \multirow[t]{2}{*}{$H$-ATLAS J125632.7+233625 } & $\mathrm{NC}$ & 70 & & & & & & & 1.5 \\
\hline & & & & & & & & & 1.5 \\
\hline \multirow[t]{2}{*}{$H$-ATLAS J132630.1+334410 } & & & $+33:$ & & & & & & 3.8 \\
\hline & & & & & & & & & \\
\hline \multirow[t]{2}{*}{$H$-ATLAS J132859.3+292327 } & NA & & 07 & & & & & & 2.3 \\
\hline & & & & & & & & & 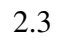 \\
\hline$H$-ATLAS J133008.4+2 & & 56 & +24 & & & & & & 4. \\
\hline$H$-ATLAS J084933.4+021443 & $\begin{array}{l}\text { G09v } \\
\text { G09v }\end{array}$ & & + & $\begin{array}{l}\text { 08: } \\
08:\end{array}$ & & 1 & 7 & 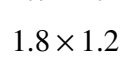 & \\
\hline & & & & & & & & & \\
\hline & & & & & & & & & 3.8 \\
\hline & & & & & & & & & 7.7 \\
\hline$H-A$ & & & 34.05 & $13: 44: 29.46$ & 4.01 & & 227.828 & $1.7 \times 1.7$ & 2.3 \\
\hline
\end{tabular}

Notes. RA and Dec are the J2000 Herschel coordinates which were taken as the centres of the NOEMA images displayed in Fig. A.1; RA $\mathrm{pk}_{\text {and }}$ $\mathrm{Dec}_{\mathrm{pk}}$ are the $\mathrm{J} 2000$ coordinates of the NOEMA dust continuum image peaks; $v_{\mathrm{obs}}$ is the central observed frequency. The rest-frame frequencies of para- $\mathrm{H}_{2} \mathrm{O} 2_{02}-1_{11}, 2_{11}-2_{02}$ and ortho- $\mathrm{H}_{2} \mathrm{O} 3_{21}-3_{12}$ lines are: $987.927 \mathrm{GHz}, 752.033 \mathrm{GHz}$ and $1162.912 \mathrm{GHz}$, respectively (the rest-frame frequencies are taken from the JPL catalogue: http://spec.jpl.nasa.gov); $t_{\mathrm{on}}$ is the on-source integration time. The source G09v1.124, which is not resolved by SPIRE, is a cluster that consists of two main components: eastern component W (G09v1.124-W) and western component T (G09v1.124-T) as described in Ivison et al. (2013) (see also Fig. A.3).

Table 2. Previously observed properties of the sample.

\begin{tabular}{|c|c|c|c|c|c|c|c|c|c|c|c|}
\hline Source & $z$ & $\begin{array}{l}F_{250} \\
(\mathrm{mJy})\end{array}$ & $\begin{array}{l}F_{350} \\
(\mathrm{mJy})\end{array}$ & $\begin{array}{l}F_{500} \\
(\mathrm{mJy})\end{array}$ & $\begin{array}{l}F_{880} \\
(\mathrm{mJy})\end{array}$ & $\begin{array}{l}r_{\text {half }} \\
(\mathrm{kpc})\end{array}$ & $\frac{\Sigma_{\mathrm{SFR}}}{\left(10^{3} M_{\odot} \mathrm{yr}^{-1} \mathrm{kpc}^{-2}\right)}$ & $\begin{array}{r}f_{1.4 \mathrm{GHz}} \\
(\mathrm{mJy})\end{array}$ & $\begin{array}{c}T_{\mathrm{d}} \\
(\mathrm{K})\end{array}$ & $\mu$ & $\begin{array}{c}\mu L_{\mathrm{IR}} \\
\left(10^{13} L_{\odot}\right)\end{array}$ \\
\hline G09v1.97 & 3.634 & $260 \pm 7$ & $321 \pm 8$ & $269 \pm 9$ & $85.5 \pm 4.0$ & 0.85 & $0.91 \pm 0.15$ & \pm 0.15 & $44 \pm 1$ & $6.9 \pm 0.6$ & $15.3 \pm 4.3$ \\
\hline G12v2.43 & 3.127 & $290 \pm 7$ & $295 \pm 8$ & $216 \pm 9$ & $48.6 \pm 2.3$ & - & - & \pm 0.15 & - & - & $(8.3 \pm 1.7)$ \\
\hline NCv1.143 & 3.565 & $214 \pm 7$ & $291 \pm 8$ & $261 \pm 9$ & $97.2 \pm 6.5$ & 0.40 & $2.08 \pm 0.77$ & $0.61 \pm 0.16$ & $40 \pm 1$ & $11.3 \pm 1.7$ & $12.8 \pm 4.3$ \\
\hline NAv1.195 & 2.951 & $179 \pm 7$ & $279 \pm 8$ & $265 \pm 9$ & $65.2 \pm 2.3$ & 1.57 & $0.21 \pm 0.04$ & \pm 0.14 & $36 \pm 1$ & $4.1 \pm 0.3$ & $7.4 \pm 2.0$ \\
\hline NAv1.177 & 2.778 & $264 \pm 9$ & $310 \pm 10$ & $261 \pm 10$ & $50.1 \pm 2.1$ & - & - & \pm 0.15 & - & _- & $(5.5 \pm 1.1)$ \\
\hline NBv1.78 & 3.111 & $273 \pm 7$ & $282 \pm 8$ & $214 \pm 9$ & $59.2 \pm 4.3$ & 0.55 & $1.09 \pm 1.41$ & $0.67 \pm 0.20$ & $43 \pm 1$ & $13.0 \pm 1.5$ & $10.7 \pm 3.9$ \\
\hline$\overline{G 09 v 1.124-W^{a}}$ & 2410 & & & & 5 & - & - & \pm 0.15 & $40 \pm 1$ & 1 & $3.3 \pm 0.3$ \\
\hline G09v1.124-T ${ }^{a}$ & 2.410 & & & & & - & - & \pm 0.15 & $36 \pm 1$ & $1.5 \pm 0.2$ & $2.7 \pm 0.8$ \\
\hline G09v1.40 & $2.089^{b}$ & $389 \pm 7$ & $381 \pm 8$ & $241 \pm 9$ & $61.4 \pm 2.9$ & 0.41 & $0.77 \pm 0.30$ & $0.75 \pm 0.15$ & $36 \pm 1$ & $15.3 \pm 3.5$ & $6.5 \pm 2.5$ \\
\hline SDP11 & 1.786 & $417 \pm 6$ & $378 \pm 7$ & $232 \pm 8$ & $30.6 \pm 2.4$ & 0.89 & $0.22 \pm 0.08$ & $0.66 \pm 0.14$ & $41 \pm 1$ & $10.9 \pm 1.3$ & $6.2 \pm 1.9$ \\
\hline NCv1.268 & 3.675 & $145 \pm 7$ & $201 \pm 8$ & $212 \pm 9$ & $78.9 \pm 4.4$ & 0.93 & $0.31 \pm 0.14$ & $1.10 \pm 0.14$ & $39 \pm 1$ & $11.0 \pm 1.0$ & $9.5 \pm 2.7$ \\
\hline NAv1.56 & 2.301 & $481 \pm 9$ & $484 \pm 13$ & $344 \pm 11$ & $73.1 \pm 2.4$ & 1.50 & $0.14 \pm 0.08$ & $1.12 \pm 0.27$ & $38 \pm 1$ & $11.7 \pm 0.9$ & $11.3 \pm 3.1$ \\
\hline
\end{tabular}

Notes. $z$ is the redshift inferred from previous $\mathrm{CO}$ detection quoted by B13 (see the references therein); $F_{250}, F_{350}$ and $F_{500}$ are the SPIRE flux densities at 250,350 and $500 \mu \mathrm{m}$, respectively (Pascale et al. 2011); $F_{880}$ is the SMA flux density at $880 \mu \mathrm{m} ; r_{\text {half }}$ and $\Sigma_{\mathrm{SFR}}$ are the intrinsic half-light radius at $880 \mu \mathrm{m}$ and the lensing-corrected surface SFR (star formation rate) density (Sect. 4.2); $f_{1.4 \mathrm{GHz}}$ is the $1.4 \mathrm{GHz}$ band flux densities from the VLA FIRST survey; $T_{\mathrm{d}}$ is the cold-dust temperature taken from B13 (note that the errors quoted for $T_{\mathrm{d}}$ are significantly underestimated since the uncertainties from differential lensing and single-temperature dust SED assumption are not fully considered); $\mu$ is the lensing magnification factor from B13, except for G09v1.124 which is adopted from Ivison et al. (2013); $\mu L_{\mathrm{IR}}$ is the apparent total infrared luminosity mostly inferred from $\mathrm{B} 13$. The $\mu L_{\mathrm{IR}}$ in brackets are not listed in B13, thus we infer them from single modified black body dust SED fitting using the submm photometry data listed in this table. ${ }^{(a)}$ The cluster source G09v1.124 includes two main components: G09v1.124-W to the east and G09v1.124-T to the west (Fig. A.3) and the values of these two rows are quoted from Ivison et al. (2013); ${ }^{(b)}$ our $\mathrm{H}_{2} \mathrm{O}$ observation gives $z=2.093$ for G09v1.40. This value is slightly different from the value of 2.089 quoted by B13 from Lupu et al. (in prep.) obtained by CSO/Z-Spec, but consistent with CO(3-2) observation by Riechers et al. (in prep.).

\subsection{General properties of the $\mathrm{H}_{2} \mathrm{O}$ emissions}

To measure the linewidth, velocity-integrated flux density and the continuum level of the spectra from the source peak and from the entire source, we extract each spectrum from the CLEANed image at the position of the source peak in a single synthesis beam and the spectrum integrated over the entire source. Then we fit them with Gaussian profiles using MPFIT (Markwardt 2009).

We detect the high-excitation ortho- $\mathrm{H}_{2} \mathrm{O}\left(3_{21}-3_{12}\right)$ in five out of six observed sources, with high signal to noise ratios
$(S / N>9)$ and velocity-integrated flux densities comparable to those of the low-excitation $J=2$ para- $\mathrm{H}_{2} \mathrm{O}$ lines (Table 4 and Figs. 2 and A.1). We also detect nine out of eleven $J=2$ para$\mathrm{H}_{2} \mathrm{O}$ lines, either $2_{02}-1_{11}$ or $2_{11}-2_{02}$, with $S / N \geq 6$ in terms of their velocity-integrated flux density, plus one tentative detection of $\mathrm{H}_{2} \mathrm{O}\left(2_{02}-1_{11}\right)$ in SDP11. We present the values of velocityintegrated $\mathrm{H}_{2} \mathrm{O}$ flux density detected at the source peak in a single synthesised beam, $I_{\mathrm{H}_{2}} \mathrm{O}$ pk , and the velocity-integrated $\mathrm{H}_{2} \mathrm{O}$ flux density over the entire source, $I_{\mathrm{H}_{2} \mathrm{O}}$ (Table 4 ). The detected $\mathrm{H}_{2} \mathrm{O}$ lines are strong, with $\mathrm{I}_{\mathrm{H}_{2} \mathrm{O}}=3.7-14.6 \mathrm{Jy} \mathrm{km} \mathrm{s}^{-1}$. Even considering gravitational lensing correction, this is consistent with 

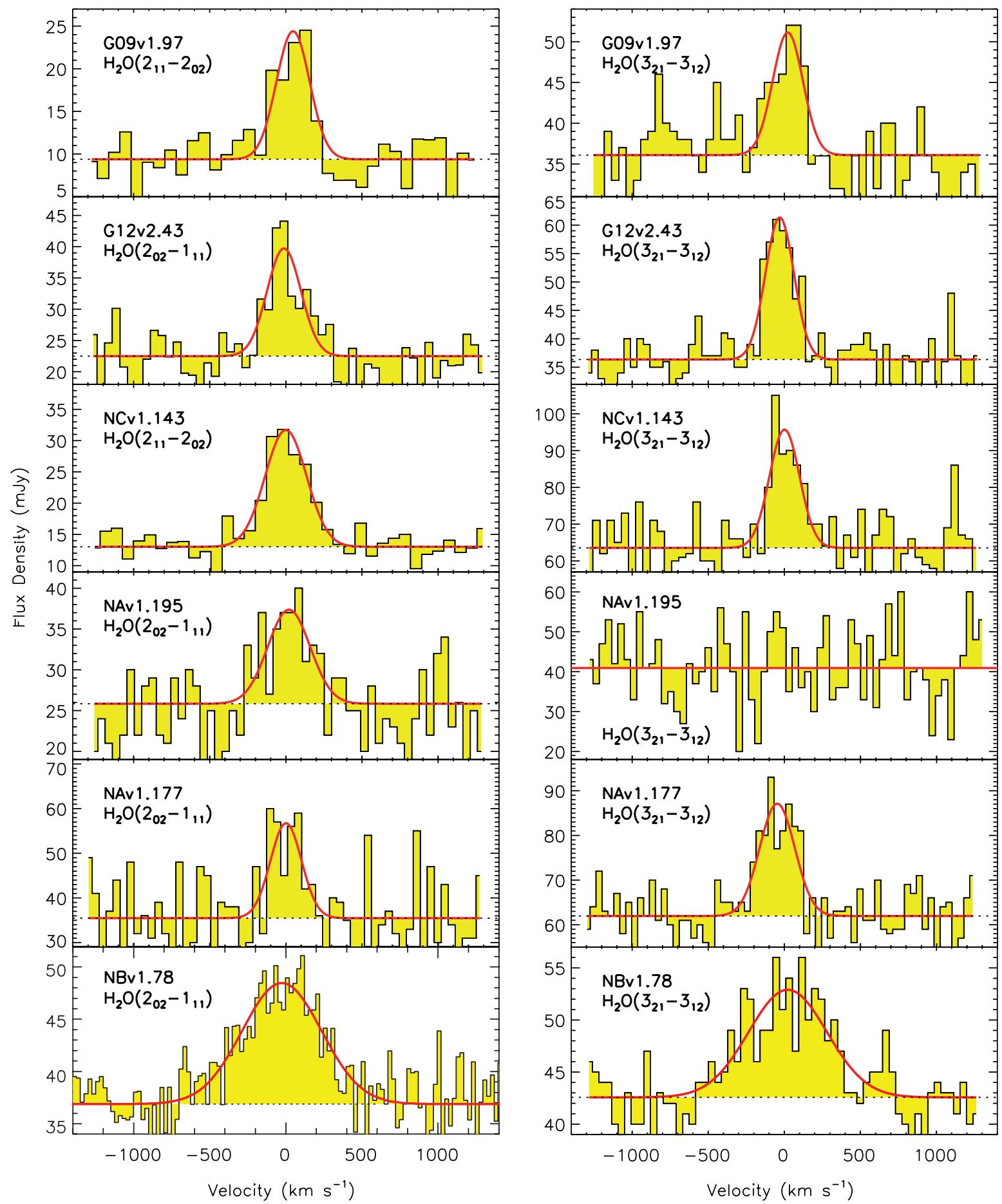

Fig. 2a. Spatially integrated spectra of $\mathrm{H}_{2} \mathrm{O}$ in the six sources with both $J=2$ para- $\mathrm{H}_{2} \mathrm{O}$ and $J=3$ ortho- $\mathrm{H}_{2} \mathrm{O}$ lines observed. The red lines represent the Gaussian fitting to the emission lines. The $\mathrm{H}_{2} \mathrm{O}\left(2_{02}-1_{11}\right)$ spectrum of $\mathrm{NBv} 1.78$ is taken from $\mathrm{O} 13$. Except for $\mathrm{H}_{2} \mathrm{O}\left(3_{21}-3_{12}\right)$ in NAv1.195, all the $J=2$ and $J=3 \mathrm{H}_{2} \mathrm{O}$ lines are well detected, with a high $\mathrm{S} / \mathrm{N}$ and similar profiles in both lines for the same source.

our previous finding that high-redshift Hy/ULIRGs are very strong $\mathrm{H}_{2} \mathrm{O}$ emitters, with $\mathrm{H}_{2} \mathrm{O}$ flux density approaching that of CO (Tables 3 and 4 and Sect.4.3). The majority of the images ( $7 / 11$ for $J=2$ lines and $3 / 4$ for $J=3$ ) are marginally resolved with $I_{\mathrm{H}_{2} \mathrm{O}}$ pk $/ I_{\mathrm{H}_{2} \mathrm{O}} \sim 0.4-0.7$. They show somewhat lensed structures. The others are unresolved with $I_{\mathrm{H}_{2} \mathrm{O}} \mathrm{pk} / I_{\mathrm{H}_{2} \mathrm{O}}>0.8$. All continuum emission flux densities $\left(S_{\nu}(\mathrm{ct})^{\mathrm{pk}}\right.$ for the emission peak and $S_{v}(\mathrm{ct})$ for the entire source) are very well detected $(S / N \geq 30)$, with a range of total flux density of 9-64 mJy for $S_{v}(\mathrm{ct})$. Figure A.1 shows the low-resolution images of $\mathrm{H}_{2} \mathrm{O}$ and the corresponding dust continuum emission at the observing frequencies. Because the positions of the sources were derived from Herschel observation, which has a large beamsize (>17") comparing to the source size, the position of most of the sources are not perfectly centred at these Herschel positions as seen in the maps. The offsets are all within the position error of the Herschel measurement (Fig. A.1). G09v1.124 is a complex HyLIRG system including two main components eastern G09v1.124-W and western G09v1.124-T as described in Ivison et al. (2013). In Fig. A.3, we identified the two strong components separated 

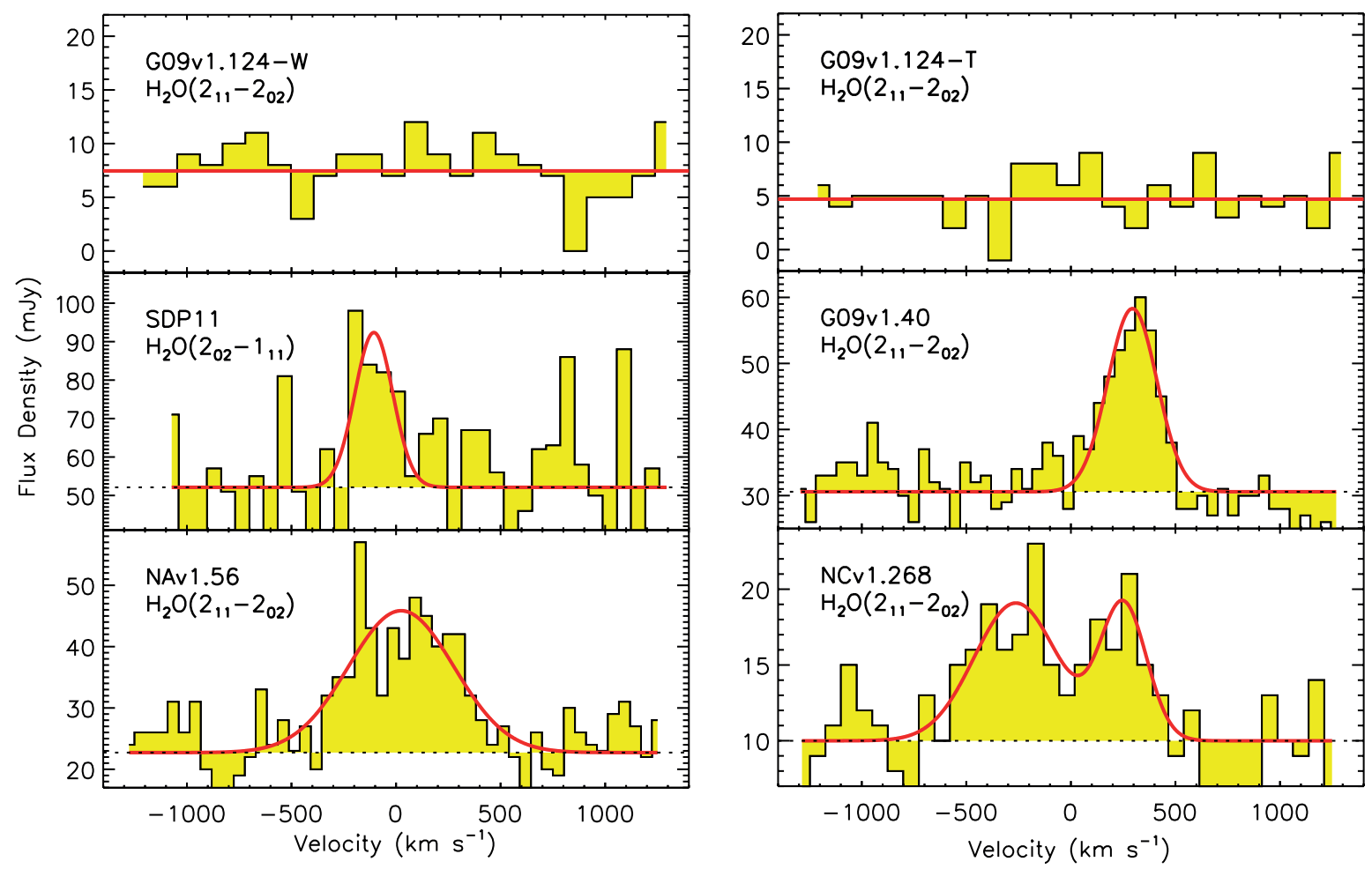

Fig. 2b. Spatially integrated spectra of $\mathrm{H}_{2} \mathrm{O}$ of the five sources with only one $J=2$ para- $\mathrm{H}_{2} \mathrm{O}$ line observed. The red lines represent the Gaussian fitting to the emission lines. Except for the $\mathrm{H}_{2} \mathrm{O}$ line in $\mathrm{G} 09 \mathrm{v} 1.124$, all the $J=2 \mathrm{H}_{2} \mathrm{O}$ lines are well detected.

Table 3. Observed CO line properties using the IRAM $30 \mathrm{~m} / \mathrm{EMIR}$.

\begin{tabular}{lrrr}
\hline \hline Source & CO line & $\begin{array}{c}I_{\mathrm{CO}} \\
\left(\mathrm{Jy} \mathrm{km} \mathrm{s}^{-1}\right)\end{array}$ & $\begin{array}{c}\Delta V_{\mathrm{CO}} \\
\left(\mathrm{km} \mathrm{s}^{-1}\right)\end{array}$ \\
\hline G09v1.97 & $5-4$ & $9.5 \pm 1.2$ & $224 \pm 32$ \\
& $6-5$ & $10.4 \pm 2.3$ & $292 \pm 86$ \\
NCv1.143 & $5-4$ & $13.1 \pm 1.0$ & $273 \pm 27$ \\
& $6-5$ & $11.0 \pm 1.0$ & $284 \pm 27$ \\
NAv1.195 & $5-4$ & $11.0 \pm 0.6$ & $281 \pm 16$ \\
NAv1.177 & $3-2$ & $6.8 \pm 0.4$ & $231 \pm 15$ \\
& $5-4$ & $11.0 \pm 0.6$ & $230 \pm 16$ \\
NBv1.78 & $5-4$ & $10.3 \pm 0.8$ & $614 \pm 53$ \\
& $6-5$ & $9.7 \pm 1.0$ & $734 \pm 85$ \\
G09v1.40 & $4-3$ & $7.5 \pm 2.1$ & $198 \pm 51$ \\
NAv1.56 & $5-4$ & $17.7 \pm 6.6$ & $432 \pm 182$ \\
\hline
\end{tabular}

Notes. $I_{\mathrm{CO}}$ is the velocity-integrated flux density of $\mathrm{CO} ; \Delta V_{\mathrm{CO}}$ is the linewidth (FWHM) derived from fitting a single Gaussian to the line profile.

about $10^{\prime \prime}$, in agreement with Ivison et al. (2013). The $J=2$ $\mathrm{H}_{2} \mathrm{O}$ and dust continuum emissions in NBv1.78, NCv1.195, G09v1.40, SDP 11 and NAv1.56, as well as the $J=3$ ortho- $\mathrm{H}_{2} \mathrm{O}$ and the corresponding dust continuum emissions in G09v1.97, NCv1.143 and NAv1.177, are marginally resolved as shown in Fig. A.1. Their images are consistent with the corresponding SMA images (B13) in terms of their spatial distribution. The rest of the sources are not resolved by the low-resolution synthesised beams. The morphological structure of the $\mathrm{H}_{2} \mathrm{O}$ emission is similar to the continuum for most sources as shown in Fig. A.1. The ratio $S_{v}(\mathrm{ct})^{\mathrm{pk}} / S_{v}(\mathrm{ct})$ and $S_{v}\left(\mathrm{H}_{2} \mathrm{O}\right)^{\mathrm{pk}} / S_{v}\left(\mathrm{H}_{2} \mathrm{O}\right)$ are in good agreement within the error. However, for $\mathrm{NCv} 1.143$ in which $S_{v}(\mathrm{ct})^{\mathrm{pk}} / S_{v}(\mathrm{ct})=0.55 \pm 0.01$ and $S_{v}\left(\mathrm{H}_{2} \mathrm{O}\right)^{\mathrm{pk}} / S_{v}\left(\mathrm{H}_{2} \mathrm{O}\right)=$ $0.74 \pm 0.16$, the $J=3$ ortho- $\mathrm{H}_{2} \mathrm{O}$ emission appears more compact than the dust continuum. Generally it seems unlikely that we have a significant fraction of missing flux for our sources. Nevertheless, the low angular resolution $\left(\sim 1^{\prime \prime}\right.$ at best) limits the study of spatial distribution of the gas and dust in our sources. A detailed analysis of the images for each source is given in Appendix A.

The majority of the sources have $\mathrm{H}_{2} \mathrm{O}$ (and $\mathrm{CO}$ ) linewidths between 210 and $330 \mathrm{~km} \mathrm{~s}^{-1}$, while the four others range between 500 and $700 \mathrm{~km} \mathrm{~s}^{-1}$ (Table 4). Except NCv1.268, which shows a double-peaked line profile, all $\mathrm{H}_{2} \mathrm{O}$ lines are well fit by a single Gaussian profile (Fig. 2). The line profiles between the $J=2$ and $J=3 \mathrm{H}_{2} \mathrm{O}$ lines do not seem to be significantly different, as shown from the linewidth ratios ranging from $1.26 \pm 0.14$ to $0.84 \pm 0.16$. The magnification from strong lensing is very sensitive to the spatial configuration, in other words, differential lensing, which could lead to different line profiles if the different velocity components of the line are emitted at different spatial positions. Since there is no visible differential effect between their profiles, it is possible that the $J=2$ and $J=3 \mathrm{H}_{2} \mathrm{O}$ lines are from similar spatial regions.

In addition to $\mathrm{H}_{2} \mathrm{O}$, within the $3.6 \mathrm{GHz}$ WideX band, we have also tentatively detected $\mathrm{H}_{2} \mathrm{O}^{+}$emission in 3 sources: $\mathrm{NCv} 1.143$, G09v1.97 and G15v2.779 (see Sect. 5).

\subsection{Lensing properties}

All our sources are strongly gravitationally lensed (except G09v1.124, see Appendix A.11), which increases the line flux densities and allows us to study the $\mathrm{H}_{2} \mathrm{O}$ emission in an affordable amount of observation time. However, the complexity of the lensed images complicates the analysis. As mentioned above, most of our lensed images are either unresolved or marginally resolved. Thus, we will not discuss here the spatial distribution of the $\mathrm{H}_{2} \mathrm{O}$ and dust emissions through gravitational lensing modelling. However, we should keep in mind that the correction of 
Table 4. Observed properties of $\mathrm{H}_{2} \mathrm{O}$ emission lines.

\begin{tabular}{|c|c|c|c|c|c|c|c|c|c|c|}
\hline Source & $\mathrm{H}_{2} \mathrm{O}$ line & $\begin{array}{l}v_{\mathrm{H}_{2} \mathrm{O}} \\
(\mathrm{GHz})\end{array}$ & $\begin{array}{c}S_{v}(\mathrm{ct})^{\mathrm{pk}} \\
\left(\frac{\mathrm{mJy}}{\text { beam }}\right)\end{array}$ & $\begin{array}{l}S_{v}(\mathrm{ct}) \\
(\mathrm{mJy})\end{array}$ & $\begin{array}{c}S_{\mathrm{H}_{2} \mathrm{O}}^{\mathrm{pk}} \\
\left(\frac{\mathrm{mJy}}{\text { beam }}\right)\end{array}$ & $\begin{array}{r}S_{\mathrm{H}_{2} \mathrm{O}} \\
(\mathrm{mJy})\end{array}$ & $\begin{array}{c}I_{\mathrm{H}_{2} \mathrm{O}^{\mathrm{pk}}} \\
\left(\frac{\mathrm{y} \mathrm{km} \mathrm{s}^{-1}}{\text { beam }}\right) \\
\end{array}$ & $\begin{array}{c}I_{\mathrm{H}_{2} \mathrm{O}} \\
\left(\mathrm{Jy} \mathrm{km} \mathrm{s}^{-1}\right)\end{array}$ & $\begin{array}{c}\Delta V_{\mathrm{H}_{2} \mathrm{O}} \\
\left(\mathrm{km} \mathrm{s}^{-1}\right)\end{array}$ & $\begin{array}{c}\mu L_{\mathrm{H}_{2} \mathrm{O}} \\
\left(10^{8} L_{\odot}\right)\end{array}$ \\
\hline \multirow[t]{2}{*}{ G09v1.97 } & $22_{11}-2_{02}$ & 162.255 & $8.9 \pm 0.2$ & $9.4 \pm 0.2$ & $14.9 \pm 2.2$ & $15.0 \pm 2.1$ & $3.8 \pm 0.4$ & $4.1 \pm 0.4$ & $257 \pm 27$ & $7.4 \pm 0.7$ \\
\hline & $3_{21}-3_{12}$ & 250.947 & $21.7 \pm 0.3$ & $36.1 \pm 0.3$ & $7.8 \pm 1.9$ & $15.0 \pm 2.6$ & $2.4 \pm 0.4$ & $3.7 \pm 0.4$ & $234 \pm 34$ & $10.4 \pm 1.0$ \\
\hline \multirow[t]{2}{*}{ G12v2.43 } & $2_{02}-1_{11}$ & 239.388 & $16.0 \pm 0.3$ & $22.5 \pm 0.4$ & $10.8 \pm 2.1$ & $17.3 \pm 3.1$ & $3.2 \pm 0.5$ & $4.8 \pm 0.6$ & $262 \pm 35$ & $8.8 \pm 1.0$ \\
\hline & $3_{21}-3_{12}$ & 281.784 & $31.5 \pm 0.3$ & $36.4 \pm 0.3$ & $25.6 \pm 3.3$ & $25.0 \pm 3.0$ & $4.9 \pm 0.4$ & $5.9 \pm 0.5$ & $221 \pm 20$ & $12.7 \pm 1.0$ \\
\hline \multirow[t]{2}{*}{ NCv1.143 } & $2_{11}-2_{02}$ & 164.741 & $11.2 \pm 0.1$ & $13.3 \pm 0.2$ & $17.4 \pm 1.3$ & $18.7 \pm 1.3$ & $5.6 \pm 0.3$ & $5.8 \pm 0.3$ & $293 \pm 15$ & $10.1 \pm 0.5$ \\
\hline & $3_{21}-3_{12}$ & 254.739 & $34.8 \pm 0.5$ & $63.5 \pm 0.5$ & $23.9 \pm 4.3$ & $32.1 \pm 4.1$ & $5.2 \pm 0.6$ & $8.0 \pm 0.7$ & $233 \pm 22$ & $21.3 \pm 1.8$ \\
\hline \multirow[t]{2}{*}{ NAv1.195 } & $2_{02}-1_{11}$ & 250.034 & $14.0 \pm 0.4$ & $25.8 \pm 0.4$ & $6.6 \pm 2.5$ & $11.6 \pm 2.5$ & $2.1 \pm 0.6$ & $4.0 \pm 0.6$ & $328 \pm 51$ & $6.7 \pm 1.0$ \\
\hline & $3_{21}-3_{12}$ & (293.334) & $17.2 \pm 0.5$ & $41.2 \pm 0.5$ & $<4.2$ & $<7.3$ & $<1.5$ & $<2.6$ & $330^{a}$ & $<5.0$ \\
\hline \multirow[t]{2}{*}{ NAv1.177 } & $2_{02}-1_{11}$ & 261.489 & $26.5 \pm 0.6$ & $35.5 \pm 0.6$ & $16.8 \pm 4.9$ & $21.2 \pm 4.9$ & $4.4 \pm 0.9$ & $5.4 \pm 0.9$ & $241 \pm 41$ & $8.2 \pm 1.2$ \\
\hline & $3_{21}-3_{12}$ & 307.856 & $38.2 \pm 0.4$ & $62.0 \pm 0.4$ & $14.8 \pm 2.6$ & $25.2 \pm 3.1$ & $4.6 \pm 0.5$ & $7.3 \pm 0.6$ & $272 \pm 24$ & $12.9 \pm 1.1$ \\
\hline \multirow[t]{2}{*}{$\mathrm{NBv} 1.78$} & $2_{02}-1_{11}^{b}$ & 240.290 & $15.4 \pm 0.3$ & $36.9 \pm 0.4$ & $5.0 \pm 1.0$ & $12.3 \pm 3.2$ & $2.7 \pm 0.3$ & $6.7 \pm 1.3$ & $510 \pm 90$ & $12.2 \pm 2.4$ \\
\hline & $3_{21}-3_{12}$ & 282.863 & $29.2 \pm 0.2$ & $42.6 \pm 0.2$ & $8.8 \pm 1.0$ & $10.6 \pm 1.0$ & $4.8 \pm 0.4$ & $6.7 \pm 0.5$ & $607 \pm 43$ & $14.3 \pm 1.0$ \\
\hline G09v1.124-W & \multirow{2}{*}{$2_{11}-2_{02}$} & \multirow{2}{*}{ (220.537) } & $6.42 \pm 0.15$ & $7.6 \pm 0.2$ & $<1.4$ & $<1.6$ & $<1.2^{c}$ & $<1.4^{c}$ & $850^{c}$ & $<1.3^{c}$ \\
\hline G09v1.124-T & & & $4.08 \pm 0.15$ & $4.9 \pm 0.2$ & $<1.7$ & $<2.0$ & $<1.0^{c}$ & $<1.2^{c}$ & $550^{c}$ & $<1.0^{c}$ \\
\hline G09v1.40 & $2_{11}-2_{02}$ & 243.182 & $16.9 \pm 0.2$ & $30.6 \pm 0.3$ & $17.5 \pm 2.0$ & $27.7 \pm 1.9$ & $4.9 \pm 0.4$ & $8.2 \pm 0.4$ & $277 \pm 14$ & $5.7 \pm 0.3$ \\
\hline SDP11 & $2_{02}-1_{11}$ & 354.930 & $29.2 \pm 1.3$ & $52.1 \pm 1.3$ & $14.8 \pm 8.4$ & $40.3 \pm 11.7$ & $5.2 \pm 2.0$ & $9.2 \pm 2.0$ & $214 \pm 41$ & $6.3 \pm 1.1$ \\
\hline NCv1.268 & $2_{11}-2_{02}$ & 161.013 & $6.6 \pm 0.1$ & $10.0 \pm 0.1$ & $5.2 \pm 1.1$ & $9.0 \pm 1.2$ & $3.7 \pm 0.4$ & $7.0 \pm 0.7$ & $731 \pm 75$ & $12.8 \pm 1.2$ \\
\hline NAv1.56 & $2_{11}-2_{02}$ & 227.822 & $14.0 \pm 0.6$ & $22.7 \pm 0.6$ & $15.8 \pm 3.3$ & $23.2 \pm 3.0$ & $7.8 \pm 1.1$ & $14.6 \pm 1.3$ & $593 \pm 56$ & $12.0 \pm 1.1$ \\
\hline
\end{tabular}

Notes. $v_{\mathrm{H}_{2} \mathrm{O}}$ is the observed central frequency of $\mathrm{H}_{2} \mathrm{O}$ lines, and the values in brackets are the $\mathrm{H}_{2} \mathrm{O}$ line frequencies inferred from the CO redshifts for the undetected sources; $S_{v}(\mathrm{ct})^{\mathrm{pk}}$ and $S_{v}(\mathrm{ct})$ are the peak and spatially integrated continuum flux density, respectively; $S_{\mathrm{H}_{2} \mathrm{O}}^{\mathrm{pk}}$ is the peak $\mathrm{H}_{2} \mathrm{O}$ line flux and $S_{\mathrm{H}_{2} \mathrm{O}}$ is the total line flux; $I_{\mathrm{H}_{2} \mathrm{O}}$ pk and $I_{\mathrm{H}_{2} \mathrm{O}}$ are the peak and spatially integrated velocity-integrated flux density of the $\mathrm{H}_{2} \mathrm{O}$ lines; $\Delta V_{\mathrm{H}_{2} \mathrm{O}}$ is the $\mathrm{H}_{2} \mathrm{O}$ linewidth; $\mu L_{\mathrm{H}_{2} \mathrm{O}}$ is the apparent luminosity of the observed $\mathrm{H}_{2} \mathrm{O}$ line. ${ }^{(a)}$ The linewidth of the undetected $\mathrm{H}_{2} \mathrm{O}\left(3_{21}-3_{12}\right)$ in NAv1.195 has been set to $330 \mathrm{~km} \mathrm{~s}^{-1}$ by assuming that the widths of the $\mathrm{H}_{2} \mathrm{O}\left(3_{21}-3_{12}\right)$ and $\mathrm{H}_{2} \mathrm{O}\left(2_{02}-1_{11}\right)$ lines are roughly the same; ${ }^{(b)}$ the data of para- $\mathrm{H}_{2} \mathrm{O}\left(2_{02}-1_{11}\right)$ in $\mathrm{NBv} 1.78$ is taken from $\mathrm{O} 13 ;{ }^{(c)}$ the $2 \sigma$ upper limits of $I_{\mathrm{H}_{2} \mathrm{O}}$ are derived by assuming that the $\mathrm{H}_{2} \mathrm{O}$ linewidths are similar to those of the CO lines (Ivison et al. 2013).

the magnification is a crucial part of our study. In addition, differential lensing could have a significant influence when comparing $\mathrm{H}_{2} \mathrm{O}$ emission with dust and even comparing different transitions of same molecular species (Serjeant 2012), especially for the emission from close to the caustics.

In order to infer the intrinsic properties of our sample, especially $L_{\mathrm{H}_{2} \mathrm{O}}$ as in our first paper O13, we adopted the lensing magnification factors $\mu$ (Table 2) computed from the modelling of the $880 \mu \mathrm{m}$ SMA images (B13). As shown in the Appendix, the ratio of $S_{v}(\mathrm{ct})^{\mathrm{pk}} / S_{v}(\mathrm{ct})$ and $S_{v}\left(\mathrm{H}_{2} \mathrm{O}\right)^{\mathrm{pk}} / S_{v}\left(\mathrm{H}_{2} \mathrm{O}\right)$ are in good agreement within the uncertainties. Therefore, it is unlikely that the magnification of the $880 \mu$ m continuum image and $\mathrm{H}_{2} \mathrm{O}$ can be significantly different. However, B13 were unable to provide a lensing model for two of our sources, G12v2.43 and NAv1.177, because their lens deflector is unidentified. This does not affect the modelling of $\mathrm{H}_{2} \mathrm{O}$ excitation and the comparison of $\mathrm{H}_{2} \mathrm{O}$ and infrared luminosities since the differential lensing effect seems to be insignificant as discussed in Sects. 4 and Appendix A.

\section{Discussion}

\section{1. $L_{\mathrm{H}_{2} \mathrm{O}}-L_{I R}$ correlation and $L_{\mathrm{H}_{2} \mathrm{O}}-L_{I R}$ ratio}

Using the formula given by Solomon et al. (1992), we derive the apparent $\mathrm{H}_{2} \mathrm{O}$ luminosities of the sources, $\mu L_{\mathrm{H}_{2} \mathrm{O}}$ (Table 4), from $I_{\mathrm{H}_{2} \mathrm{O}}$. For the ortho- $\mathrm{H}_{2} \mathrm{O}\left(3_{21}-3_{12}\right)$ lines, $\mu L_{\mathrm{H}_{2} \mathrm{O}}$ varies in the range of $6-22 \times 10^{8} L_{\odot}$, while the $\mu L_{\mathrm{H}_{2} \mathrm{O}}$ of the $J=2$ lines are a factor $\sim 1.2-2$ weaker (Table 4) as discussed in Sect. 4.2 .

Using the lensing magnification correction (taking the values of $\mu$ from B13), we have derived the intrinsic $\mathrm{H}_{2} \mathrm{O}$ luminosities (Table 5). The error of each luminosity consists of the uncertainty from both observation and the gravitational lensing modelling. After correcting for lensing, the $\mathrm{H}_{2} \mathrm{O}$ luminosities of our high-redshift galaxies appear to be one order of magnitude higher than those of local ULIRGs, as well as their infrared luminosities (Table 5), so that many of them should rather be considered as HyLIRGs than ULIRGs. Though the ratio of $L_{\mathrm{H}_{2} \mathrm{O}} / L_{\mathrm{IR}}$ in our high-redshift sample is close to that of local ULIRGs (Y13), with somewhat a statistical increase in the extreme high $L_{\mathrm{IR}}$ end (Fig. 3).

As displayed in Fig. 3 for $\mathrm{H}_{2} \mathrm{O}$ of the three observed lines, because we have extended the number of detections to $21 \mathrm{H}_{2} \mathrm{O}$ lines, distributed in 16 sources and 3 transitions, we may independently study the correlation of $L_{\mathrm{H}_{2} \mathrm{O}\left(2_{02}-1_{11}\right)}$ and $L_{\mathrm{H}_{2} \mathrm{O}\left(2_{11}-2_{02}\right)}$ with $L_{\mathrm{IR}}$, while we had approximately combined the two lines in O13.

As found in $\mathrm{O} 13$, the correlation is slightly steeper than linear $\left(L_{\mathrm{H}_{2} \mathrm{O}} \sim L_{\mathrm{IR}}^{1.2}\right)$. To broaden the dynamical range of this comparison, we also included the local ULIRGs from Y13, together with a few other $\mathrm{H}_{2} \mathrm{O}$ detections in high-redshift Hy/ULIRGs, for example, HLSJ 0918 (HLSJ 091828.6+514223) (Combes et al. 2012; Rawle et al. 2014), APM 08279 (van der Werf et al. 2011), SPT 0538 (SPT-S J0538165030.8) (Bothwell et al. 2013) and HFLS3 (Riechers et al. 2013, with the magnification factor from Cooray et al. 2014) (Fig. 3). In the fitting, however, we excluded the sources with heavy AGN contamination (Mrk231 and APM 08279) or missing flux resolved out by the interferometry (SDP 81). We also excluded the $\mathrm{H}_{2} \mathrm{O}\left(3_{21}-3_{12}\right)$ line of HFLS3

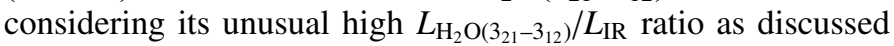
above, that could bias our fitting. We have performed a linear regression in log-log space using the Metropolis-Hastings Markov Chain Monte Carlo (MCMC) algorithm sampler through linmix_err (Kelly 2007) to derived the $\alpha$ in

$L_{\mathrm{H}_{2} \mathrm{O}} \propto L_{\mathrm{IR}}^{\alpha}$ 
Table 5. IR luminosity, $\mathrm{H}_{2} \mathrm{O}$ line luminosity and global dust temperature of the entire sample.

\begin{tabular}{|c|c|c|c|c|c|}
\hline Source & $\mathrm{H}_{2} \mathrm{O}$ Transition & $\begin{array}{l}L_{\mathrm{IR}} \\
\left(10^{12} L_{\odot}\right)\end{array}$ & $\begin{array}{c}L_{\mathrm{H}_{2} \mathrm{O}\left(2_{11}-2_{02}\right)} \\
\left(10^{7} L_{\odot}\right)\end{array}$ & $\begin{array}{c}L_{\mathrm{H}_{2} \mathrm{O}\left(2_{02}-1_{11}\right)} \\
\left(10^{7} L_{\odot}\right)\end{array}$ & $\begin{array}{c}L_{\mathrm{H}_{2} \mathrm{O}\left(3_{21}-3_{12}\right)} \\
\left(10^{7} L_{\odot}\right)\end{array}$ \\
\hline G09v1.97 & $2_{11}-2_{02}, 3_{21}-3_{12}$ & $22.1 \pm 5.9$ & $10.7 \pm 1.4$ & - & $15.0 \pm 1.9$ \\
\hline G12v2.43 & $2_{02}-1_{11}, 3_{21}-3_{12}$ & $83.2 \pm 16.6 / \mu$ & - & $88.4 \pm 10.7 / \mu$ & $143.2 \pm 11.5 / \mu$ \\
\hline NCv1.143 & $22_{11}-2_{02}, 3_{21}-3_{12}$ & $11.4 \pm 3.1$ & $9.0 \pm 1.4$ & - & $18.9 \pm 3.3$ \\
\hline NAv1.195 & $2_{02}-1_{11}, 3_{21}-3_{12}$ & $18.0 \pm 4.6$ & - & $16.4 \pm 3.0$ & $<12.3$ \\
\hline NAv1.177 & $2_{02}-1_{11}, 3_{21}-3_{12}$ & $55.0 \pm 11.0 / \mu$ & - & $82.0 \pm 12.8 / \mu$ & $129.1 \pm 10.8 / \mu$ \\
\hline NBv1.78 & $2_{02}-1_{11}, 3_{21}-3_{12}$ & $8.2 \pm 2.2$ & - & $9.4 \pm 2.1$ & $11.0 \pm 1.5$ \\
\hline G09v1.124-W & $2_{11}-2_{02}$ & $33.1 \pm 3.2$ & $<12.9$ & - & - \\
\hline G09v1.124-T & $2_{11}-2_{02}$ & $14.5 \pm 1.8$ & $<6.9$ & - & - \\
\hline G09v1.40 & $2_{11}-2_{02}$ & $4.2 \pm 1.3$ & $3.7 \pm 0.9$ & - & - \\
\hline SDP11 & $2_{02}-1_{11}$ & $5.7 \pm 1.6$ & - & $5.8 \pm 1.4$ & - \\
\hline NCv1.268 & $2_{11}-2_{02}$ & $8.6 \pm 2.3$ & $11.5 \pm 1.5$ & - & - \\
\hline NAv1.56 & $2_{11}-2_{02}$ & $9.7 \pm 2.6$ & $10.3 \pm 1.2$ & - & - \\
\hline SDP81 & $2_{02}-1_{11}$ & 6.1 & - & 3.3 & - \\
\hline NAv1.144 & $2_{11}-2_{02}$ & 11 & 9.7 & - & - \\
\hline SDP9 & $2_{11}-2_{02}$ & 5.2 & 7.0 & - & - \\
\hline G12v2.30 & $2_{02}-1_{11}$ & 16 & - & 13 & - \\
\hline SDP17b & $2_{02}-1_{11}$ & 16 & - & 20 & - \\
\hline G15v2.779 & $2_{11}-2_{02}$ & 21 & 26.6 & - & - \\
\hline
\end{tabular}

Notes. $L_{\mathrm{IR}}$ is the intrinsic total infrared luminosity $(8-1000 \mu \mathrm{m})$ taken from B13. The intrinsic $\mathrm{H}_{2} \mathrm{O}$ luminosities are inferred from $\mu L_{\mathrm{H}_{2} \mathrm{O}}$ using $\mu$ in B13. The first group of the sources are the ones with both $J=2$ and $J=3 \mathrm{H}_{2} \mathrm{O}$ lines observed, the next group are the sources with only $J=2$ $\mathrm{H}_{2} \mathrm{O}$ observed, and the last group are the previous published sources in $\mathrm{O} 13$.

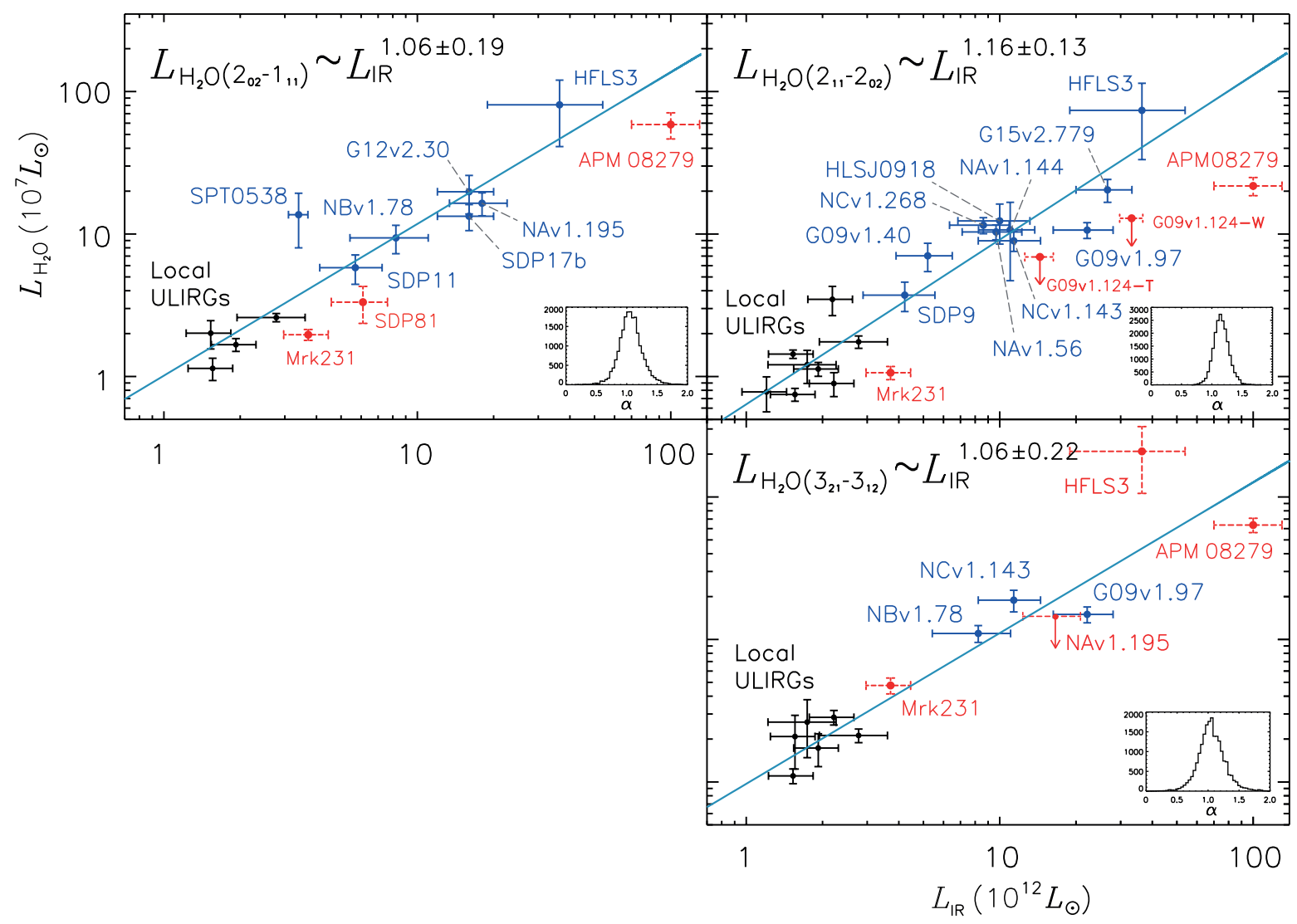

Fig. 3. Correlation between $L_{\mathrm{IR}}$ and $L_{\mathrm{H}_{2} \mathrm{O}}$ in local ULIRGs and high-redshift Hy/ULIRGs. The black points represent local ULIRGs from Y13. The blue points with solid error bars are the $H$-ATLAS source in this work together with some previously published sources. Red points with dashed error bars are excluded from the fit as described in the text. Upper limits are shown in arrows. The light blue lines show the results of the fitting. The insets are the probability density distributions of the fitted slopes $\alpha$. We find tight correlations between the luminosity of the three $\mathrm{H}_{2} \mathrm{O}$ lines and $L_{\mathrm{IR}}$, namely $L_{\mathrm{H}_{2} \mathrm{O}} \propto L_{\mathrm{IR}}^{1.1-1.2}$. 
The fitted parameters are $\alpha=1.06 \pm 0.19,1.16 \pm 0.13$ and $1.06 \pm$ 0.22 for $\mathrm{H}_{2} \mathrm{O}$ line $2_{02}-1_{11}, 2_{11}-2_{02}$ and $3_{21}-3_{12}$, respectively. Comparing with the local ULIRGs, the high-redshift lensed ones have higher $L_{\mathrm{H}_{2} \mathrm{O}} / L_{\mathrm{IR}}$ ratios (Table 6). These slopes confirm our first result derived from $7 \mathrm{H}_{2} \mathrm{O}$ detections in (O13). The slight super-linear correlations seem to indicate that far-infrared pumping play an important role in the excitation of the submm $\mathrm{H}_{2} \mathrm{O}$ emission. This is unlike the high- $J$ CO lines, which are determined by collisional excitation and follow the linear correlation between the $\mathrm{CO}$ line luminosity and $L_{\mathrm{IR}}$ from the local to the high-redshift Universe (Liu et al. 2015). As demonstrated in G14, using the far-infrared pumping model, the steeper than linear growth of $L_{\mathrm{H}_{2} \mathrm{O}}$ with $L_{\mathrm{IR}}$ can be the result of an increasing optical depth at $100 \mu \mathrm{m}\left(\tau_{100}\right)$ with increasing $L_{\mathrm{IR}}$. In local ULIRGs, the ratio of $L_{\mathrm{H}_{2} \mathrm{O}} / L_{\mathrm{IR}}$ is relatively low while most of them are likely to be optically thin $\left(\tau_{100} \sim 0.1\right.$, G14). On the other hand, for the high-redshift lensed Hy/ULIRGs with high values of $L_{\mathrm{IR}}$, the continuum optical depth at far-infrared wavelengths is expected to be high (see Sect.4.2), indicating that the $\mathrm{H}_{2} \mathrm{O}$ emission comes from very dense regions of molecular gas that are heavily obscured.

Similar to what we found in the local ULIRGs (Y13), we find again an anti-correlation between $T_{\mathrm{d}}$ and $L_{\mathrm{H}_{2} \mathrm{O}\left(3_{21}-3_{12}\right)} / L_{\mathrm{IR}}$. The Spearman's rank correlation coefficient for the five $\mathrm{H}_{2} \mathrm{O}\left(3_{21}-3_{12}\right)$ detected $H$-ATLAS sources is $\rho=-0.9$ with a two-sided significance of its deviation from zero, $p=0.04$. However, after including the non-detection of $\mathrm{H}_{2} \mathrm{O}\left(3_{21}-3_{12}\right)$ in NAv1.195, the correlation is much weaker, that is to say, $\rho \lesssim$ -0.5 and $p \sim 0.32$. No significant correlation has been found between $T_{\mathrm{d}}$ and $L_{\mathrm{H}_{2} \mathrm{O}\left(2_{02}-1_{11}\right)} / L_{\mathrm{IR}}(\rho=-0.1$ and $p=0.87)$ nor $L_{\mathrm{H}_{2} \mathrm{O}\left(2_{11}-2_{02}\right)} / L_{\mathrm{IR}}(\rho=-0.3$ and $p=0.45)$. As explained in $\mathrm{G} 14$, in the optically thick and very warm galaxies, the ratio of $L_{\mathrm{H}_{2} \mathrm{O}\left(3_{21}-3_{12}\right)} / L_{\mathrm{IR}}$ is expected to decrease with increasing $T_{\mathrm{d}}$. And this anti-correlation can not be explained by optically thin conditions. However, a larger sample is needed to increase the statistical significance of this anti-correlation.

Although, it is important to stress that the luminosity of $\mathrm{H}_{2} \mathrm{O}$ is a complex result of various physical parameters such as dust temperature, gas density, $\mathrm{H}_{2} \mathrm{O}$ abundance and $\mathrm{H}_{2} \mathrm{O}$ gas distribution relative to the infrared radiation field, etc, it is striking that the correlation between $L_{\mathrm{H}_{2} \mathrm{O}}$ and $L_{\mathrm{IR}}$ stays linear from local young stellar objects (YSOs), in which the $\mathrm{H}_{2} \mathrm{O}$ molecules are mainly excited by shocks and collisions, to local ULIRGs (far-infrared pumping dominated), extending 12 orders of magnitudes (San José-García et al. 2016), implying that $\mathrm{H}_{2} \mathrm{O}$ indeed traces the SFR proportionally, similarly to the dense gas (Gao \& Solomon 2004) in the local infrared bright galaxies. However, for the high-redshift sources, the $L_{\mathrm{H}_{2} \mathrm{O}}$ emissions are somewhat above the linear correlations which could be explained by their high $\tau_{100}$ (or large velocity dispersion). As shown in Table 6, HFLS3, with a $\tau_{100}>1$ has extremely large ratios of $L_{\mathrm{H}_{2} \mathrm{O}} / L_{\mathrm{IR}}$ which are stronger than the average of our $H$-ATLAS sources by factors $\sim 2$ for the $J=2$ lines and $\sim 4$ for $J=3$ (see Fig. 3). The velocity dispersions of its $\mathrm{H}_{2} \mathrm{O}$ lines are $\sim 900 \mathrm{~km} \mathrm{~s}^{-1}$ (with uncertainties from $18 \%$ to $36 \%$ ), which is larger than all our sources. For optically thick systems, larger velocity dispersion will increase the number of absorbed pumping photons, and boost the ratio of $L_{\mathrm{H}_{2} \mathrm{O}} / L_{\mathrm{IR}}(\mathrm{G} 14)$.

For the AGN-dominated sources (i.e. APM 08279, G09v1.124-W and Mrk231) as shown in Fig. 3, most of them (except for the $\mathrm{H}_{2} \mathrm{O}\left(3_{21}-3_{12}\right)$ line of Mrk 231) are well below the fitted correlation (see Sect. 4.4). This is consistent with the average value of local strong-AGN-dominated sources. The $J \lesssim 3 \mathrm{H}_{2} \mathrm{O}$ lines are far-infrared pumped by the 75 and $101 \mu \mathrm{m}$

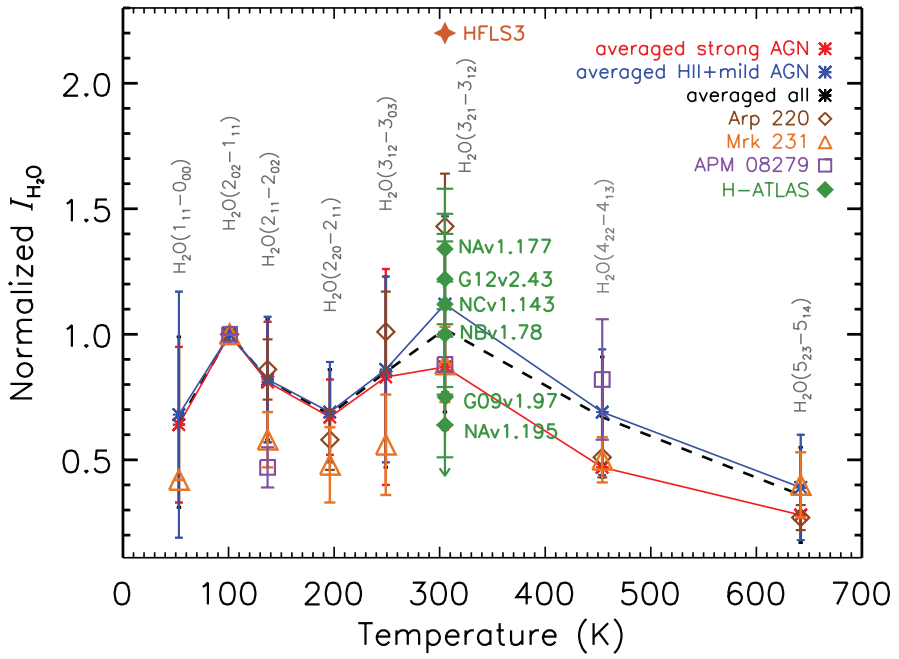

Fig. 4. Velocity-integrated flux density distribution of $\mathrm{H}_{2} \mathrm{O}$ normalised to $I_{\mathrm{H}_{2} \mathrm{O}\left(2_{02}-1_{11}\right)}$ adapted from Y13. Local averaged values are shown in black dashed line and marks. Among them, AGN-dominated sources are shown in red and star-forming dominated galaxies are shown in blue. Some individual sources are also shown in this plot as indicated by the legend. Green diamonds are the high-redshift lensed Hy/ULIRGs from this work. HFLS3 is a $z=6.3$ high-redshift galaxy from Riechers et al. (2013).

photons, thus the very warm dust in strong-AGN-dominated sources is likely to contribute more to the $L_{\mathrm{IR}}$ than the $J \lesssim 3$ $\mathrm{H}_{2} \mathrm{O}$ excitation (see also Y13).

\section{2. $\mathrm{H}_{2} \mathrm{O}$ excitation}

We have detected both $J=2$ and $J=3 \mathrm{H}_{2} \mathrm{O}$ lines in five sources out of six observed for $J=3$ ortho- $\mathrm{H}_{2} \mathrm{O}$ lines. By comparing the line ratios and their strength relative to $L_{\mathrm{IR}}$, we are able to constrain the physical conditions of the molecular content and also the properties of the far-infrared radiation field.

To compare the $\mathrm{H}_{2} \mathrm{O}$ excitation with local galaxies, we plot the velocity-integrated flux density of ortho- $\mathrm{H}_{2} \mathrm{O}\left(3_{21}-3_{12}\right)$ normalised by that of para- $\mathrm{H}_{2} \mathrm{O}\left(2_{02}-1_{11}\right)$ in our source on top of the local and high-redshift $\mathrm{H}_{2} \mathrm{O}$ SLEDs (spectral line energy distributions) in Fig. 4. All the six high-redshift sources are located within the range of the local galaxies, with a $1 \sigma$ dispersion of $\sim 0.2$. Yet for the $z=6.34$ extreme starburst HFLS3, the value of this ratio is at least 1.7 times higher than the average value of local sources (Y13) and those of our lensed high-redshift Hy/ULIRGs at $\gtrsim 3 \sigma$ confidence level (Fig. 4). This probably traces different excitation conditions, namely the properties of the dust emission, as it is suggested in G14 that the flux ratio of $\mathrm{H}_{2} \mathrm{O}\left(3_{21}-3_{12}\right)$ over $\mathrm{H}_{2} \mathrm{O}\left(2_{02}-1_{11}\right)$ is the most direct tracer of the hardness of the far-infrared radiation field which powers the submm $\mathrm{H}_{2} \mathrm{O}$ excitation. However, the line ratios are still consistent with the strong saturation limit in the far-infrared pumping model with a $T_{\text {warm }} \gtrsim 65 \mathrm{~K}$. The large scatter of the $\mathrm{H}_{2} \mathrm{O}$ line ratio between $3_{21}-3_{12}$ and $22_{02}-1_{11}$ indicates different local $\mathrm{H}_{2} \mathrm{O}$ excitation conditions. As far-infrared pumping is dominating the $\mathrm{H}_{2} \mathrm{O}$ excitation, the ratio therefore reflects the differences in the far-infrared radiation field, for example, the temperature of the warmer dust that excites the $\mathrm{H}_{2} \mathrm{O}$ gas, and the submm continuum opacity. It is now clear that far-infrared pumping is the prevailing excitation mechanism for those submm $\mathrm{H}_{2} \mathrm{O}$ lines rather than collisional excitation (G14) in infrared bright galaxies in both the local and high-redshift 
Table 6. Ratio between infrared and $\mathrm{H}_{2} \mathrm{O}$ luminosity, and the velocity-integrated flux density ratio between different $\mathrm{H}_{2} \mathrm{O}$ transitions.

\begin{tabular}{|c|c|c|c|c|c|c|c|}
\hline Source & $\mathrm{H}_{2} \mathrm{O}$ Transition & $\begin{array}{l}T_{\mathrm{d}} \\
(\mathrm{K})\end{array}$ & $\begin{array}{c}\frac{L_{\mathrm{H}_{2} \mathrm{O}\left(2_{11}-2_{02}\right)}}{L_{\mathrm{IR}}} \\
\left(\times 10^{-6}\right)\end{array}$ & $\begin{array}{c}\frac{L_{\mathrm{H}_{2} \mathrm{O}\left(2_{02}-1_{11}\right)}}{L_{\mathrm{IR}}} \\
\left(\times 10^{-6}\right)\end{array}$ & $\begin{array}{c}\frac{L_{\mathrm{H}_{2} \mathrm{O}\left(3_{21}-3_{12}\right)}}{L_{\mathrm{IR}}} \\
\left(\times 10^{-6}\right)\end{array}$ & $\frac{I_{\mathrm{H}_{2} \mathrm{O}\left(3_{21}-3_{12}\right)}}{I_{\mathrm{H}_{2}} \mathrm{O}\left(2_{11}-2_{02}\right)}$ & $\frac{I_{\mathrm{H}_{2}} \mathrm{O}\left(3_{21}-3_{12}\right)}{I_{\mathrm{H}_{2}} \mathrm{O}\left(2_{02}{ }^{-1} 11\right)}$ \\
\hline G09v1.97 & $2_{11}-2_{02}, 3_{21}-3_{12}$ & $44 \pm 1$ & $4.8 \pm 1.4$ & - & $6.8 \pm 2.0$ & $0.9 \pm 0.1$ & $(0.8 \pm 0.2)$ \\
\hline G12v2.43 & $2_{02}-1_{11}, 3_{21}-3_{12}$ & $(39 \pm 2)$ & - & $10.6 \pm 2.5$ & $15.3 \pm 3.3$ & - & $1.2 \pm 0.2$ \\
\hline NCv1.143 & $2_{11}-2_{02}, 3_{21}-3_{12}$ & $40 \pm 1$ & $7.9 \pm 2.5$ & - & $16.6 \pm 5.4$ & $1.4 \pm 0.1$ & $(1.1 \pm 0.4)$ \\
\hline NAv1.195 & $2_{02}-1_{11}, 3_{21}-3_{12}$ & $36 \pm 1$ & - & $9.1 \pm 2.9$ & $<6.8$ & - & $<0.7$ \\
\hline NAv1.177 & $2_{02}-1_{11}, 3_{21}-3_{12}$ & $(32 \pm 1)$ & - & $14.9 \pm 3.8$ & $23.5 \pm 5.1$ & - & $1.3 \pm 0.2$ \\
\hline NBv1.78 & $2_{02}-1_{11}, 3_{21}-3_{12}$ & $43 \pm 1$ & - & $11.4 \pm 4.7$ & $13.4 \pm 4.9$ & - & $1.0 \pm 0.2$ \\
\hline G09v1.124-W & $2_{11}-2_{02}$ & $40 \pm 1$ & $<3.9$ & - & - & - & - \\
\hline G09v1.124-T & $2_{11}-2_{02}$ & $36 \pm 1$ & $<4.8$ & - & - & - & - \\
\hline G09v1.40 & $2_{11}-2_{02}$ & $36 \pm 1$ & $8.8 \pm 3.5$ & - & - & - & - \\
\hline SDP11 & $2_{02}-1_{11}$ & $41 \pm 1$ & - & $10.2 \pm 3.8$ & - & - & - \\
\hline NCv1.268 & $2_{11}-2_{02}$ & $39 \pm 1$ & $13.4 \pm 3.9$ & - & - & - & - \\
\hline NAv1.56 & $2_{11}-2_{02}$ & $38 \pm 1$ & $10.7 \pm 3.1$ & - & - & - & \\
\hline SDP81 & $2_{02}-1_{11}$ & $34 \pm 1$ & - & 5.4 & - & - & - \\
\hline NAv1.144 & $2_{11}-2_{02}$ & $39 \pm 1$ & 9.7 & - & - & - & - \\
\hline SDP9 & $2_{11}-2_{02}$ & $43 \pm 1$ & 13.5 & - & - & - & - \\
\hline G12v2.30 & $2_{02}-1_{11}$ & $41 \pm 1$ & - & 8.1 & - & - & - \\
\hline SDP17b & $2_{02}-1_{11}$ & $38 \pm 1$ & - & 12.5 & - & - & - \\
\hline G15v2.779 & $2_{11}-2_{02}$ & $41 \pm 1$ & 7.7 & - & - & - & - \\
\hline HFLS3 & $2_{02}-1_{11}, 2_{11}-2_{02}, 3_{21}-3_{12}$ & $56_{-12}^{+9}$ & 20.3 & 22.2 & 57.3 & $1.8 \pm 0.6$ & $2.2 \pm 0.5$ \\
\hline APM 08279 & $2_{02}-1_{11}, 2_{11}-2_{02}, 3_{21}-3_{12}$ & $220 \pm 30$ & 2.2 & 6.0 & 6.4 & $1.9 \pm 0.3$ & $0.9 \pm 0.1$ \\
\hline HLSJ 0918 & $2_{02}-1_{11}$ & $38 \pm 3$ & 11.4 & - & - & - & - \\
\hline SPT 0538 & $2_{02}-1_{11}$ & $39 \pm 2$ & - & 40.3 & - & - & - \\
\hline local strong-AGN & $2_{02}-1_{11}, 2_{11}-2_{02}, 3_{21}-3_{12}$ & - & 3.8 & 6.4 & 6.7 & $1.1 \pm 0.4$ & $0.9 \pm 0.3$ \\
\hline local H II+mild-AGN & $2_{02}-1_{11}, 2_{11}-2_{02}, 3_{21}-3_{12}$ & - & 5.8 & 9.2 & 10.8 & $1.4 \pm 0.4$ & $1.1 \pm 0.3$ \\
\hline
\end{tabular}

Notes. The luminosity ratios between each $\mathrm{H}_{2} \mathrm{O}$ line and their total infrared, and the velocity-integrated flux density ratio of different $\mathrm{H}_{2} \mathrm{O}$ transitions. $T_{\mathrm{d}}$ is the cold-dust temperature taken from B13, except for the ones in brackets which are not listed B13, that we infer them from single modified black-body dust SED fitting using the submm $/ \mathrm{mm}$ photometry data listed in Table 2 . All the errors quoted for $T_{\mathrm{d}}$ are significantly underestimated especially because they do not include possible effects of differential lensing and make the assumption of a single-temperature. Line ratios in brackets are derived based on the average velocity-integrated flux density ratios between $2_{11}-2_{02}$ and $2_{02}-1_{11}$ lines in local infrared galaxies. The local strong-AGN sources are the optically classified AGN-dominated galaxies and the local H II+mild-AGN sources are star-forming-dominated galaxies with possible mild AGN contribution (Y13). The first group of the sources are from this work; and the sources in the second group are the previously published sources in O13; the third group contains the previously published high-redshift detections from other works: HFLS3 (Riechers et al. 2013), APM 08279 (van der Werf et al. 2011), HLSJ 0918 (Combes et al. 2012; Rawle et al. 2014) and SPT 0538 (Bothwell et al. 2013); the last group shows the local averaged values from Y13.

Universe. The main path of far-infrared pumping related to the lines we observed here are 75 and $101 \mu \mathrm{m}$ as displayed in Fig. 1. Therefore, the different line ratios are highly sensitive to the difference between the monochromatic flux at 75 and $101 \mu \mathrm{m}$. We may compare the global $T_{\mathrm{d}}$ measured from far-infrared and submm bands (B13). It includes both cold and warm dust contribution to the dust SED in the rest-frame, which is, however, dominated by cold dust observed in SPIRE bands. It is thus not surprising that we find no strong correlation between $T_{\mathrm{d}}$ and $I_{\mathrm{H}_{2} \mathrm{O}\left(3_{21}-3_{12}\right)} / I_{\mathrm{H}_{2} \mathrm{O}\left(2_{02}-1_{11}\right)}(r \sim-0.3)$. The Rayleigh-Jeans tail of the dust SED is dominated by cooler dust which is associated with extended molecular gas and less connected to the submm $\mathrm{H}_{2} \mathrm{O}$ excitation. As suggested in G14, it is indeed the warmer dust ( $T_{\text {warm }}$, as shown by the colour legend in Fig. 5) dominating at the Wien side of the dust SED that corresponds to the excitation of submm $\mathrm{H}_{2} \mathrm{O}$ lines.

To further explore the physical properties of the $\mathrm{H}_{2} \mathrm{O}$ gas content and the far-infrared dust radiation related to the submm $\mathrm{H}_{2} \mathrm{O}$ excitation, we need to model how we can infer key parameters, such as the $\mathrm{H}_{2} \mathrm{O}$ abundance and those determining the radiation properties, from the observed $\mathrm{H}_{2} \mathrm{O}$ lines. For this purpose, we use the far-infrared pumping $\mathrm{H}_{2} \mathrm{O}$ excitation model described in G14 to fit the observed $L_{\mathrm{H}_{2} \mathrm{O}}$ together with the corresponding $L_{\mathrm{IR}}$, and derive the range of continuum optical depth at $100 \mu \mathrm{m}\left(\tau_{100}\right)$, warm dust temperature $\left(T_{\text {warm }}\right)$, and $\mathrm{H}_{2} \mathrm{O}$ column density per unit of velocity interval $\left(N_{\mathrm{H}_{2} \mathrm{O}} / \Delta V\right)$ in the five sources with both $J=2$ and $J=3 \mathrm{H}_{2} \mathrm{O}$ emission detections. Due to the insufficient number of the inputs in the model, which are $L_{\mathrm{H}_{2} \mathrm{O}}$ of the two $\mathrm{H}_{2} \mathrm{O}$ lines and $L_{\mathrm{IR}}$, we are only able to perform the modelling by using the pure far-infrared pumping regime. Nevertheless, our observed line ratio between $J=3$ and $J=2 \mathrm{H}_{2} \mathrm{O}$ lines suggests that far-infrared pumping is the dominant excitation mechanism and the contribution from collisional excitation is minor (G14). The $\pm 1 \sigma$ contours from $\chi^{2}$ fitting are shown in Fig. 5 for each warm dust temperature component $\left(T_{\text {warm }}=35-115 \mathrm{~K}\right)$ per source. It is clear that with two $\mathrm{H}_{2} \mathrm{O}$ lines (one $J=2$ para- $\mathrm{H}_{2} \mathrm{O}$ and ortho- $\mathrm{H}_{2} \mathrm{O}\left(3_{12}-3_{12}\right)$ ), we will not be able to well constrain $\tau_{100}$ and $N_{\mathrm{H}_{2} \mathrm{O}} / \Delta V$. As shown in the figure, for $T_{\text {warm }} \lesssim 75 \mathrm{~K}$, both very low and very high $\tau_{100}$ could fit the observation data together with high $N_{\mathrm{H}_{2} \mathrm{O}} / \Delta V$, while the dust with $T_{\text {warm }} \gtrsim 95 \mathrm{~K}$ are likely favouring high $\tau_{100}$. In the low continuum optical depth part in Fig. 5, as $\tau_{100}$ decreases, the model needs to increase the value of $N_{\mathrm{H}_{2} \mathrm{O}} / \Delta V$ to generate sufficient $L_{\mathrm{H}_{2} \mathrm{O}}$ to be able to fit the observed $L_{\mathrm{H}_{2} \mathrm{O}} / L_{\mathrm{IR}}$. This has been observed in some local sources with low $\tau_{100}$, such as in NGC 1068 and NGC 6240. There are no absorption features in the far-infrared but submm $\mathrm{H}_{2} \mathrm{O}$ emission have been detected in these sources (G14). The important feature of such sources is 
Table 7. Parameters derived from far-infrared pumping model of $\mathrm{H}_{2} \mathrm{O}$.

\begin{tabular}{lrrrr}
\hline \hline Source & $\tau_{100}$ & $\begin{array}{c}T_{\text {warm }} \\
(\mathrm{K})\end{array}$ & $\begin{array}{r}N_{\mathrm{H}_{2} \mathrm{O}} / \Delta V \\
\left(\mathrm{~cm}^{-2} \mathrm{~km}^{-1} \mathrm{~s}\right)\end{array}$ & \multicolumn{1}{c}{$\begin{array}{c}N_{\mathrm{H}_{2} \mathrm{O}} \\
\left(\mathrm{cm}^{-2}\right)\end{array}$} \\
\hline G09v1.97 & 1.8 & $45-55$ & $(0.3-0.6) \times 10^{15}$ & $(0.3-1.1) \times 10^{17}$ \\
G12v2.43 & - & $45-95$ & $\gtrsim 0.7 \times 10^{15}$ & $\gtrsim 0.7 \times 10^{17}$ \\
NCv1.143 & 7.2 & $45-55$ & $(2.0-20) \times 10^{15}$ & $(2.0-60) \times 10^{17}$ \\
NAv1.177 & - & $45-75$ & $\gtrsim 1.0 \times 10^{15}$ & $\gtrsim 1.0 \times 10^{17}$ \\
NBv1.78 & 2.5 & $45-75$ & $\gtrsim 0.6 \times 10^{15}$ & $\gtrsim 0.6 \times 10^{17}$ \\
\hline
\end{tabular}

Notes. $\tau_{100}$ is derived from Eq. (2) with errors of a few units (see text) while $T_{\text {warm }}$ and $N_{\mathrm{H}_{2} \mathrm{O}} / \Delta V$ are inferred from the $\mathrm{H}_{2} \mathrm{O}$ excitation model $N_{\mathrm{H}_{2} \mathrm{O}}$ is calculated by taking a typical $\Delta V$ value range of $100-300 \mathrm{~km} \mathrm{~s}^{-1}$ as suggested by G14.

the lack of $J \geq 4 \mathrm{H}_{2} \mathrm{O}$ emission lines. Thus, the observation of higher excitation of $\mathrm{H}_{2} \mathrm{O}$ will discriminate between the low and high $\tau_{100}$ regimes.

Among these five sources, favoured key parameters are somewhat different showing the range of properties we can expect for such sources. Compared with the other four Hy/ULIRGs, G09v1.97 is likely to have the lowest $T_{\text {warm }}$ as only dust with $T_{\text {warm }} \sim 45-55 \mathrm{~K}$ can fit well with the data. NCv1.143 and NAv1.177 have slightly different diagnostic which yields higher dust temperature as $T_{\text {warm }} \sim 45-75 \mathrm{~K}$, while NBv1.78 and G12v2.43 tend to have the highest temperature range, $T_{\text {warm }} \sim$ $45-95 \mathrm{~K}$. The values of $T_{\text {warm }}$ are consistent with the fact that $\mathrm{H}_{2} \mathrm{O}$ traces warm gas. We did not find any significant differences between the ranges of $N_{\mathrm{H}_{2} \mathrm{O}} / \Delta V$ derived from the modelling for these five sources, although G09v1.97 tends to have lower $N_{\mathrm{H}_{2} \mathrm{O}} / \Delta V$ (Table 7). As shown in Sect. 4.4, there is no evidence of AGN domination in all our sources, the submm $\mathrm{H}_{2} \mathrm{O}$ lines likely trace the warm dust component that connect to the heavily obscured active star-forming activity. However, due to the lack of photometry data on the Wien side of the dust SEDs, we will not be able to compare the observed values of $T_{\text {warm }}$ directly with the ones derived from the modelling.

By adopting the $100 \mu \mathrm{m}$ dust mass absorption coefficient from Draine (2003) of $\kappa_{100}=27.1 \mathrm{~cm}^{2} \mathrm{~g}^{-1}$, we can derive the dust opacity by

$\tau_{100}=\kappa_{100} \sigma_{\text {dust }}=\kappa_{100}\left(\frac{M_{\text {dust }}}{A}\right)=\kappa_{100}\left(\frac{M_{\text {dust }}}{2 \pi r_{\text {half }}^{2}}\right)$

where $\sigma_{\text {dust }}$ is the dust mass column density, $M_{\text {dust }}$ is the dust mass, $A$ is the projected surface area of the dust continuum source and $r_{\text {half }}$ is the half-light radius of the source at submm. As shown in Table 2, among the five sources in Fig. 5, the values of $M_{\text {dust }}$ and $r_{\text {half }}$ in G09v1.97, NCv1.143 and NBv1.78 have been derived via gravitational lensing (B13). Consequently, the derived approximate dust optical depth at $100 \mu \mathrm{m}$ in these three sources is $\tau_{100} \approx 1.8,7.2$ and 2.5 , respectively. One should note that, the large uncertainty in both the $\kappa_{100}$ and $r_{\text {half }}$ of these high-redshift galaxies can bring a factor of few error budget. Nevertheless, by adopting a gas-to-dust mass ratio of $X=100$ (e.g. Magdis et al. 2011), we can derive the gas depletion time using the following approach,

$t_{\mathrm{dep}}=\frac{M_{\mathrm{gas}}}{S F R}=\frac{X \tau_{100}}{\Sigma_{\mathrm{SFR}} K_{100}} \approx 1.8 \times 10^{4}\left(\frac{\tau_{100}}{\frac{\Sigma_{\mathrm{SFR}}}{M_{\odot} \mathrm{yr}^{-1} \mathrm{kpc}^{-2}}}\right) \mathrm{Myr}$

where $M_{\text {gas }}$ is the total molecular gas mass and $\Sigma_{\mathrm{SFR}}$ is the surface SFR density derived from $L_{\mathrm{IR}}$ using Kennicutt (1998) calibration by assuming a Salpeter IMF (B13, and Table 2). The implied depletion time scale is $t_{\mathrm{dep}} \approx 35-60 \mathrm{Myr}$ with errors within a factor of two, in which the dominant uncertainties are from the assumed gas-to-dust mass ratio and the half-light radius. The $t_{\text {dep }}$ is consistent with the values derived from dense gas tracers, like HCN in local (U)LIRGs (e.g. Gao \& Solomon 2004; García-Burillo et al. 2012). As suggested in $\mathrm{G} 14$, the $\mathrm{H}_{2} \mathrm{O}$ and $\mathrm{HCN}$ likely to be located in the same regions, indicate that the $\mathrm{H}_{2} \mathrm{O}$ traces the dense gas as well. Thus, the $\tau_{100}$ derived above is likely also tracing the far-infrared radiation source that powers the submm $\mathrm{H}_{2} \mathrm{O}$ emissions. $\mathrm{B} 13$ also has found that these $H$-ATLAS high-redshift Hy/ULIRGs are expected to be optically thick in the far-infrared. By adding the constrain from $\tau_{100}$ above, we can better derive the physical conditions in the sources as shown in Table 7.

From their modelling of local infrared galaxies, G14 find a range of $T_{\text {warm }}=45-75 \mathrm{~K}, \tau_{100}=0.05-0.2$ and $N_{\mathrm{H}_{2} \mathrm{O}} / \Delta V=$ $(0.5-2) \times 10^{15} \mathrm{~cm}^{-2} \mathrm{~km}^{-1} \mathrm{~s}$. The modelling results for our high-redshift sources are consistent with those in local galaxies in terms of $T_{\text {warm }}$ and $N_{\mathrm{H}_{2} \mathrm{O}} / \Delta V$. However, the values of $\tau_{100}$ we found at high-redshift are higher than those of the local infrared galaxies. This is consistent with the higher ratio between $L_{\mathrm{H}_{2} \mathrm{O}}$ and $L_{\mathrm{IR}}$ at high-redshift (Y13) which could be explained by higher $\tau_{100}$ (G14). However, as demonstrated in an extreme sample, a very large velocity dispersion will also increase the value of $L_{\mathrm{H}_{2} \mathrm{O}} / L_{\mathrm{IR}}$ within the sources with $\tau_{100}>1$. Thus, the higher ratio can also be explained by larger velocity dispersion (not including systemic rotations) in the high-redshift Hy/ULIRGs. Compared with local ULIRGs, our $H$-ATLAS sources are much more powerful in terms of their $L_{\mathrm{IR}}$. The dense warm gas regions that $\mathrm{H}_{2} \mathrm{O}$ traces are highly obscured with much more powerful far-infrared radiation fields, which possibly are close to the limit of maximum starbursts. Given the values of dust temperature and dust opacity, the radiation pressure $P_{\text {rad }} \sim \tau_{100} \sigma T_{\mathrm{d}} / c$ ( $\sigma$ is Stefan-Boltzmann's constant and $c$ the speed of light) of our sources is about $0.8 \times 10^{-7} \mathrm{erg} \mathrm{cm}^{-3}$. If we assume a $\mathrm{H}_{2}$ density $n_{\mathrm{H}_{2}}$ of $\sim 10^{6} \mathrm{~cm}^{-3}$ and take $T_{\mathrm{k}} \sim 150 \mathrm{~K}$ as suggested in G14, the thermal pressure $P_{\mathrm{th}} \sim n_{\mathrm{H}_{2}} k_{\mathrm{B}} T_{\mathrm{k}} \sim 2 \times 10^{-8} \mathrm{erg} \mathrm{cm}^{-3}\left(k_{\mathrm{B}}\right.$ is the Boltzmann constant and $T_{\mathrm{k}}$ is the gas temperature). Assuming a turbulent velocity dispersion of $\sigma_{\mathrm{v}} \sim 20-50 \mathrm{~km} \mathrm{~s}^{-1}$ (Bournaud et al. 2015) and taking molecular gas mass density $\rho \sim 2 \mu n_{\mathrm{H}_{2}}$ ( $2 \mu$ is the average molecular mass) would yield for the turbulent pressure $P_{\text {turb }} \sim \rho \sigma_{\mathrm{v}}^{2} / 3 \sim 4 \times 10^{-6} \mathrm{erg} \mathrm{cm}^{-3}$. This might be about an order of magnitude larger than $P_{\text {rad }}$ and two orders of magnitude larger than $P_{\text {th }}$, but we should note that all values are very uncertain, especially $P_{\text {turb }}$ which could be uncertain by, at maximum, a factor of a few tens. Therefore, keeping in mind their large uncertainties, turbulence and/or radiation are likely to play an important role in limiting the star formation.

\subsection{Comparison between $\mathrm{H}_{2} \mathrm{O}$ and $\mathrm{CO}$}

The velocity-integrated flux density ratio between submm $\mathrm{H}_{2} \mathrm{O}$ and submm CO lines with comparable frequencies is $0.02-0.03$ in local PDRs such as Orion and M 82 (Weiß et al. 2010). But this ratio in local ULIRGs (Y13) and in $H$-ATLAS high-redshift Hy/ULIRGs is much higher, from 0.4 to 1.1 (Tables 3 and 4). The former case is dominated by typical PDRs, where CO lines are much stronger than $\mathrm{H}_{2} \mathrm{O}$ lines, while the latter sources shows clearly a different excitation regime, in which $\mathrm{H}_{2} \mathrm{O}$ traces the central core of warm, dense and dusty molecular gas which is about a few hundred parsec (González-Alfonso et al. 2010) in diameter in local ULIRGs and highly obscured even at far-infrared. 


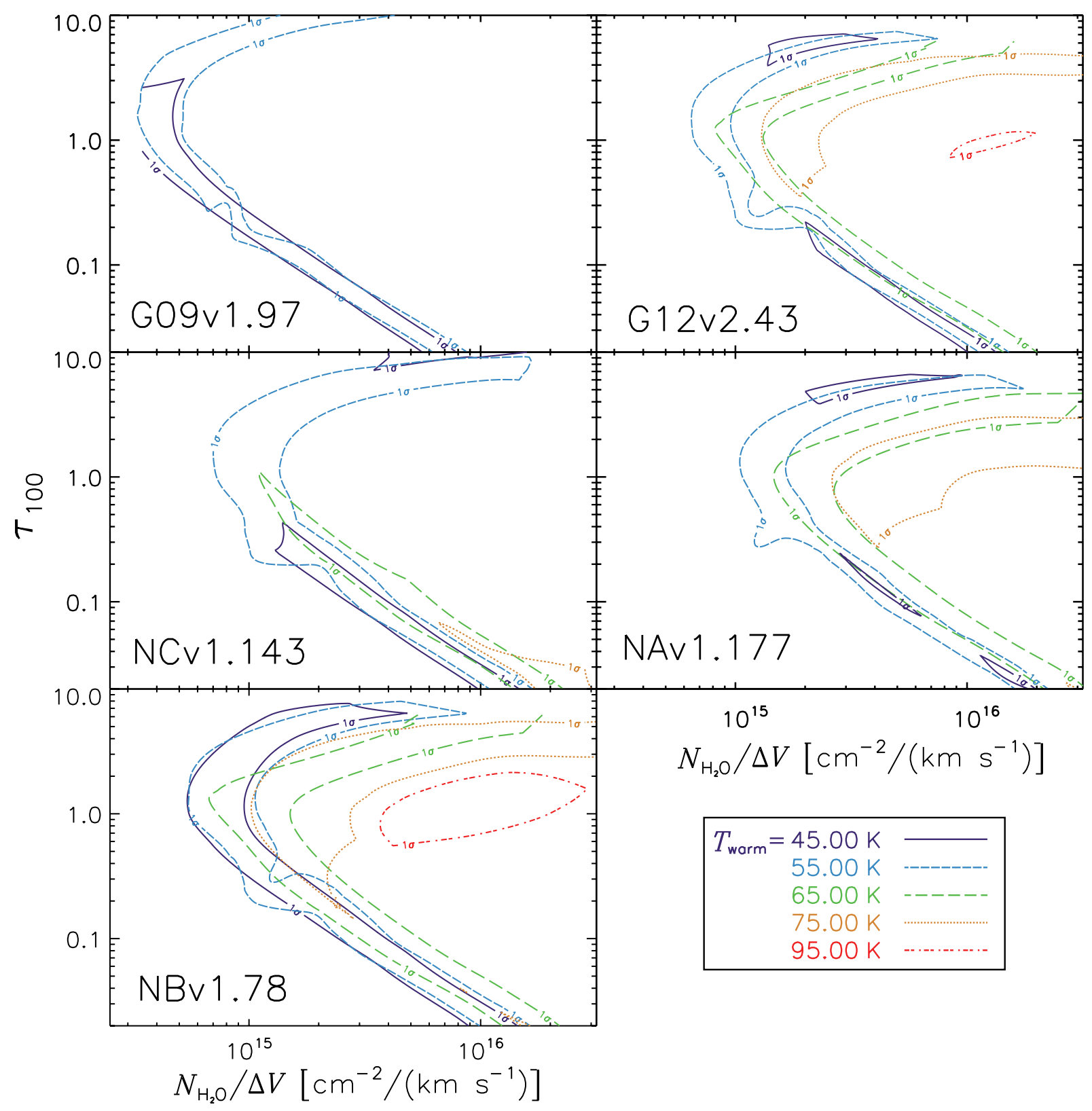

Fig. 5. Parameter space distribution of the $\mathrm{H}_{2} \mathrm{O}$ far-infrared pumping excitation modelling with observed para- $\mathrm{H}_{2} \mathrm{O} 2_{02}-1_{11}$ or $2_{11}-2_{02}$ and ortho$\mathrm{H}_{2} \mathrm{O}\left(3_{21}-3_{12}\right)$ in each panel. $\pm 1 \sigma$ contours are shown for each plot. Different colours with different line styles represent different temperature components of the warm dust as shown in the legend. The explored warm dust temperature range is from $35 \mathrm{~K}$ to $115 \mathrm{~K}$. The temperature contours that are unable to fit the data are not shown in this figure. From the figure, we are able to constrain the $\tau_{100}, T_{\text {warm }}$ and $N_{\mathrm{H}_{2} \mathrm{O}} / \Delta V$ for the five sources. However, there are strong degeneracies. Thus, we need additional information, such as the velocity-integrated flux densities of $J \geq 4 \mathrm{H}_{2} \mathrm{O}$ lines, to better constrain the physical parameters.

Generally, submm $\mathrm{H}_{2} \mathrm{O}$ lines are dominated by far-infrared pumping that traces strong far-infrared dust continuum emission, which is different from the regime of molecular gas traced by collisional excited $\mathrm{CO}$ lines. In the active star-forming nucleus of the infrared-bright galaxies, the far-infrared pumped $\mathrm{H}_{2} \mathrm{O}$ is expected to trace directly the far-infrared radiation generated by the intense star formation, which can be well correlated with the high- $J$ CO lines (Liu et al. 2015). Thus there is likely to be a correlation between the submm $\mathrm{H}_{2} \mathrm{O}$ and $\mathrm{CO}$ emission. From our previous observations, most of the $\mathrm{H}_{2} \mathrm{O}$ and $\mathrm{CO}$ line profiles are quite similar from the same source in our high-redshift lensed Hy/ULIRGs sample (Fig. 2 of O13). In the present work, we again find similar profiles between $\mathrm{H}_{2} \mathrm{O}$ and $\mathrm{CO}$ in terms of their FWHM with an extended sample (Table 3 and 4). In both cases the FWHMs of $\mathrm{H}_{2} \mathrm{O}$ and $\mathrm{CO}$ are generally equal within typical $1.5 \sigma$ errors (see special discussion for each source in Appendix A).

As the gravitational lensing magnification factor is sensitive to spatial alignment, the similar line profiles could thus suggest similar spatial distributions of the two gas tracers. However, there are a few exceptional sources, such as SDP 81 (ALMA Partnership, Vlahakis et al. 2015) and HLSJ0918 (Rawle et al. 2014). In both cases, the $\mathrm{H}_{2} \mathrm{O}$ lines are lacking the blue velocity component found in the $\mathrm{CO}$ line profiles. Quite different from the rest sources, in SDP 81 and HLSJ0918, the CO line profiles are complicated with multiple velocity components. Moreover, the velocity-integrated flux density ratios between these $\mathrm{CO}$ components may vary following 
the excitation level (different $J_{\text {up }}$ ). Thus, it is important to analyse the relation between different $\mathrm{CO}$ excitation components (from low- $J$ to high- $J$ ) and $\mathrm{H}_{2} \mathrm{O}$. Also, high resolution observation is needed to resolve the multiple spatial gas components and compare the $\mathrm{CO}$ emission with $\mathrm{H}_{2} \mathrm{O}$ and dust continuum emission within each component.

\subsection{AGN content}

It is still not clear how a strong AGN could affect the excitation of submm $\mathrm{H}_{2} \mathrm{O}$ in both local ULIRGs and high-redshift Hy/ULIRGs. Nevertheless, there are some individual studies addressing this question. For example, in APM 08279, van der Werf et al. (2011) found that AGN is the main power source that excites the high- $J \mathrm{H}_{2} \mathrm{O}$ lines and also enriches the gas-phase $\mathrm{H}_{2} \mathrm{O}$ abundance. Similar conclusion has also been drawn by González-Alfonso et al. (2010) that in Mrk 231 the AGN accounts for at least $50 \%$ contribution to the far-infrared radiation that excites $\mathrm{H}_{2} \mathrm{O}$. From the systematic study of local sources (Y13), slightly lower values of $L_{\mathrm{H}_{2} \mathrm{O}} / L_{\mathrm{IR}}$ are found in strong-AGN-dominated sources. In the present work, the decreasing ratio of $L_{\mathrm{H}_{2} \mathrm{O}} / L_{\mathrm{IR}}$ with $\mathrm{AGN}$ is clearly shown in Fig. 3 where Mrk 231, G09v1.124-W and APM 08279 are below the correlation by factors between 2 and 5 with less than $30 \%$ uncertainties (except the $\mathrm{H}_{2} \mathrm{O}\left(3_{21}-1_{23}\right)$ line of Mrk 231).

In the far-infrared pumping regime, the buried AGN will provide a strong far-infrared radiation source that will pump the $\mathrm{H}_{2} \mathrm{O}$ lines. However, the very warm dust powered by the AGN will increase the value of $L_{\mathrm{IR}}$ faster than the number of $\geq 75 \mu \mathrm{m}$ photons that is dominating the excitation of $J \leq 3 \mathrm{H}_{2} \mathrm{O}$ lines (e.g. Kirkpatrick et al. 2015). If we assume that the strength of the $\mathrm{H}_{2} \mathrm{O}$ emission is proportional to the number of pumping photons, then in the strong-AGN-dominated sources, the ratio of $L_{\mathrm{H}_{2} \mathrm{O}} / L_{\mathrm{IR}}$ will decrease since much warmer dust is present. Moreover, strong radiation from the AGN could dissociate the $\mathrm{H}_{2} \mathrm{O}$ molecules.

To evaluate the AGN contribution to the $H$-ATLAS sources, we extracted the $1.4 \mathrm{GHz}$ radio flux from the FIRST radio survey (Becker et al. 1995) listed in Table 2. By comparing the far-infrared and radio emission using the $q$ parameter (Condon $1992), q \equiv \log \left(L_{\mathrm{FIR}} / 3.75 \times 10^{12} \mathrm{~W}\right)-\log \left(L_{1.4 \mathrm{GHz}} / 1 \mathrm{~W} \mathrm{~Hz}^{-1}\right)$, we derive values of $q$ from 1.9 to 2.5 in our sources. These values follow the value $2.3 \pm 0.1$ found by Yun et al. (2001) for non strong-radio AGN. This may suggest that there is also no significant indication of a high radio contribution from AGN. This is also confirmed by the Wide-field Infrared Survey Explorer (WISE, Wright et al. 2010), which does not detect our sources at $12 \mu \mathrm{m}$ and $22 \mu \mathrm{m}$. However, rest-frame optical spectral observations show that G09v1.124-W is rather a powerful AGN (Oteo et al., in prep.), which is the only identified AGN-dominated source in our sample.

\section{Detection of $\mathrm{H}_{2} \mathrm{O}^{+}$emission lines}

$\mathrm{H}_{2} \mathrm{O}$ can be formed through both solid-state and gas-phase chemical reactions (van Dishoeck et al. 2013). On dust-grain mantles, surface chemistry dominates the formation of $\mathrm{H}_{2} \mathrm{O}$ molecules. Then they can be released into the ISM gas through sublimation. In the gas phase, $\mathrm{H}_{2} \mathrm{O}$ can be produced through two routes: the neutral-neutral reaction, usually related to shocks, creates $\mathrm{H}_{2} \mathrm{O}$ via $\mathrm{O}+\mathrm{H}_{2} \longrightarrow \mathrm{OH}+\mathrm{H}$; $\mathrm{OH}+\mathrm{H}_{2} \longrightarrow \mathrm{H}_{2} \mathrm{O}+\mathrm{H}$ at high temperature $(\gtrsim 300 \mathrm{~K})$. At lower temperature $(\lesssim 100 \mathrm{~K})$, the ion-neutral reactions in photon-dominated regions (PDRs), cosmic-ray-dominated regions and $\mathrm{X}$-ray-dominated regions (e.g. Meijerink \& Spaans 2005) generate $\mathrm{H}_{2} \mathrm{O}$ from $\mathrm{O}, \mathrm{H}^{+}, \mathrm{H}_{3}^{+}$ and $\mathrm{H}_{2}$, with intermediates such as $\mathrm{O}^{+}, \mathrm{OH}^{+}, \mathrm{H}_{2} \mathrm{O}^{+}$and $\mathrm{H}_{3} \mathrm{O}^{+}$, and finally $\mathrm{H}_{3} \mathrm{O}^{+}+\mathrm{e} \longrightarrow \mathrm{H}_{2} \mathrm{O}+\mathrm{H}$. However, classical PDRs are not likely linked to these highly excited submm $\mathrm{H}_{2} \mathrm{O}$ emissions (Y13). Therefore, $\mathrm{H}_{2} \mathrm{O}^{+}$lines are important for distinguishing between shock- or ion-chemistry origin for $\mathrm{H}_{2} \mathrm{O}$ in the early Universe, indicating the type of physical regions in these galaxies: shock-dominated regions, cosmic-ray-dominated regions or Xray-dominated regions. Indeed, they can be among the most direct tracers of the cosmic-ray or/and X-ray ionization rate (e.g. Gérin et al. 2010; Neufeld et al. 2010; González-Alfonso et al. 2013) of the ISM, which regulates the chemistry and influences many key parameters, for example, X-factor (Bell et al. 2007) that connects the $\mathrm{CO}$ luminosity to the $\mathrm{H}_{2}$ mass. Moreover, the significant detections of $\mathrm{H}_{2} \mathrm{O}^{+}$emission in high-redshift $\mathrm{Hy} / \mathrm{ULIRG}$ could help us understanding $\mathrm{H}_{2} \mathrm{O}$ formation in the early Universe.

When observing our sources with redshift $z \gtrsim 3.3$, it is possible to cover all the following lines with the NOEMA WideX bandwidth: para- $\mathrm{H}_{2} \mathrm{O}\left(2_{11}-2_{02}\right)$ at $752 \mathrm{GHz}$ and four ortho- $\mathrm{H}_{2} \mathrm{O}^{+}$lines (two intertwined fine structure doublets of two different lines whose frequencies almost coincide by chance): $2_{02}-1_{11(5 / 2-3 / 2)}$ at $742.1 \mathrm{GHz}, 22_{11}-2_{02(5 / 2-3 / 2)}$ at $742.3 \mathrm{GHz}$, $2_{02}-1_{11(3 / 2-3 / 2)}$ at $746.3 \mathrm{GHz}$ and $2_{11}-2_{02(5 / 2-5 / 2)}$ at $746.5 \mathrm{GHz}$, in the $3.6 \mathrm{GHz}$ band simultaneously (the rest-frame frequencies are taken from the CDMS catalogue: http://www . astro. uni-koeln.de/cdms, see energy level diagram of $\mathrm{H}_{2} \mathrm{O}^{+}$in Fig. 1 and the full spectra in Fig. 6). Additionally, within this range, we can also cover the $\mathrm{H}_{2}^{18} \mathrm{O}\left(2_{11}-2_{02}\right)$ line at $745.3 \mathrm{GHz}$. There are three sources of our sample that have been observed in such a frequency setup: NCv1.143, NCv1.268 and G09v1.97. We have also included the source G15v2.779 from our previous observation (O13), in which we have covered both $\mathrm{H}_{2} \mathrm{O}\left(2_{11}-2_{02}\right)$ at $752 \mathrm{GHz}$ and $\mathrm{H}_{2} \mathrm{O}^{+}$lines around $746 \mathrm{GHz}$. We have detected both main lines of $\mathrm{H}_{2} \mathrm{O}^{+}$in $\mathrm{NCv} 1.143$, and tentatively detected one line in G09v1.97 and G15v2.779 (Fig. 6). For NCv1.268, due to the large noise level and the complex line profile, we were not able to really identify any $\mathrm{H}_{2} \mathrm{O}^{+}$line detection.

As shown in Fig. 6, in NCv1.143, the dominant $\mathrm{H}_{2} \mathrm{O}^{+}$fine structure lines $2_{11}-2_{02}(5 / 2-5 / 2)$ at $746.5 \mathrm{GHz}$ and $2_{02}-1_{11(5 / 2-3 / 2)}$ at $742.1 \mathrm{GHz}$ are well detected. The velocity-integrated flux densities of the two lines from a two-Gaussian fit are $1.9 \pm 0.3$ and $1.6 \pm 0.2 \mathrm{Jy} \mathrm{km} \mathrm{s}^{-1}$, respectively. These are the approximate velocity-integrated flux densities of the dominant $\mathrm{H}_{2} \mathrm{O}^{+}$ lines $2_{11}-2_{02}(5 / 2-5 / 2)$ and $2_{02}-1_{11}(5 / 2-3 / 2)$ if neglecting the minor contributions from $\mathrm{H}_{2} \mathrm{O}^{+}$lines $2_{02}-1_{11(3 / 2-3 / 2)}$ at $746.2 \mathrm{GHz}$ and $2_{11}-2_{02}(5 / 2-3 / 2)$ at $742.3 \mathrm{GHz}$. However, the $\mathrm{H}_{2} \mathrm{O}^{+}$line profile at $746.5 \mathrm{GHz}$ is slightly wider than the $\mathrm{H}_{2} \mathrm{O}$ line (Fig. 6), probably due to a contribution from the fairly weak fine structure line $\mathrm{H}_{2} \mathrm{O}^{+}\left(2_{02}-1_{11}\right)_{(3 / 2-3 / 2)}$ at $746.3 \mathrm{GHz}$. The ratio between total velocity-integrated flux density of the $\mathrm{H}_{2} \mathrm{O}^{+}$lines and $\mathrm{H}_{2} \mathrm{O}\left(2_{11}-2_{02}\right)$ is $0.60 \pm 0.07$ (roughly 0.3 for each dominant $\mathrm{H}_{2} \mathrm{O}^{+}$line), being consistent with the average value from the local infrared galaxies (Y13) ${ }^{3}$. In order to derive the velocityintegrated flux density of each fine structure doublets around 742 and $746 \mathrm{GHz}$, we have also performed a four-Gaussian fit with fixed line positions (equal to $\left.v_{\text {rest }} /(1+z)\right)$ and linewidth (equals to that of $\mathrm{H}_{2} \mathrm{O}\left(2_{11}-2_{02}\right)$ ). We find the velocity-integrated

\footnotetext{
3 As suggested by González-Alfonso et al. (2013), due to the very limited spectral resolution of Herschel/SPIRE FTS, the ortho$\mathrm{H}_{2} \mathrm{O}^{+}\left(2_{02}-1_{11}\right)_{(3 / 2-3 / 2)}$ line at $746.5 \mathrm{GHz}$ quoted in $\mathrm{Y} 13$ is actually dominated by ortho- $\mathrm{H}_{2} \mathrm{O}^{+}\left(2_{11}-2_{02}\right)_{(5 / 2-5 / 2)}$, considering their likely excitation and relative strength.
} 

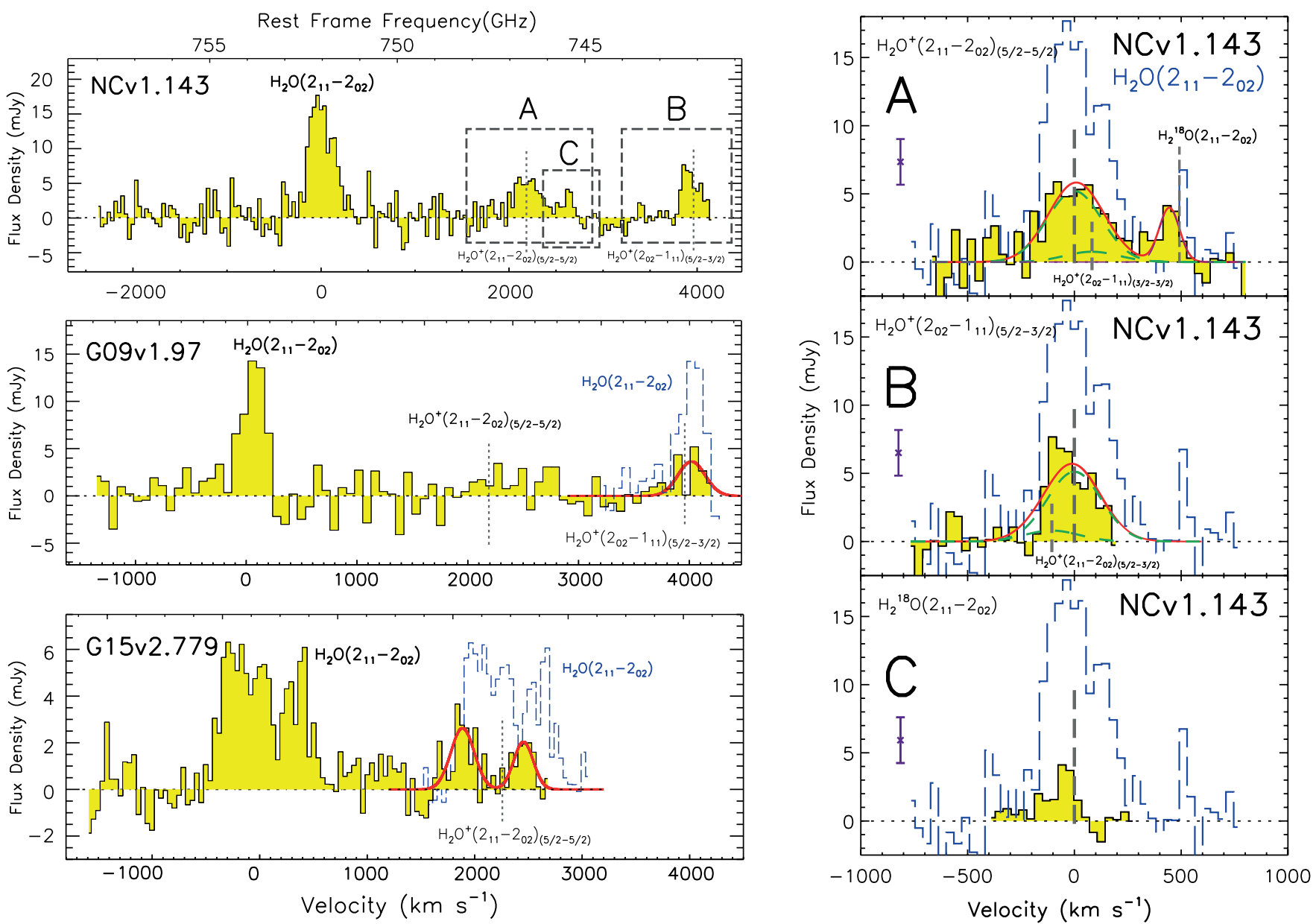

Fig. 6. Left panel: from top to bottom are the full NOEMA spectrum at $v_{\text {rest }} \sim 750 \mathrm{GHz}$ of NCv1.143, G09v1.97 and G15v2.779, respectively. The reference frequency is the redshifted frequency of the line $\mathrm{H}_{2} \mathrm{O}\left(2_{11}-2_{02}\right)$. The frequencies of the main $\mathrm{H}_{2} \mathrm{O}^{+}\left(2_{11}-2_{02}\right)_{(5 / 2-5 / 2)}$ and $\mathrm{H}_{2} \mathrm{O}^{+}\left(2_{02}-1_{11}\right)_{(5 / 2-3 / 2)}$ lines are indicated by grey vertical dashed lines. The three dashed squares in the spectrum of NCv1.143 show the position of each zoom-in spectrum of the $\mathrm{H}_{2} \mathrm{O}^{+}$(or the $\mathrm{H}_{2}^{18} \mathrm{O}$ ) as displayed in the right panel indicated by the $\mathrm{A}, \mathrm{B}$ or $\mathrm{C}$. The superposed blue dashed histograms represents the spectra of $\mathrm{H}_{2} \mathrm{O}\left(2_{11}-2_{02}\right.$ ) centred at the frequencies of the $\mathrm{H}_{2} \mathrm{O}^{+}$lines. Note that, in many cases, the observed frequency ranges (yellow histograms) do not include the full expected profiles for the $\mathrm{H}_{2} \mathrm{O}^{+}$lines. The red curve represents the Gaussian fitting to the spectra. We have detected both $\mathrm{H}_{2} \mathrm{O}^{+}$lines in NCv1.143, and tentatively detected $\mathrm{H}_{2} \mathrm{O}^{+}\left(2_{02}-1_{11}\right)_{(5 / 2-3 / 2)}$ in $\mathrm{G} 09 \mathrm{v} 1.97$ and $\mathrm{H}_{2} \mathrm{O}^{+}\left(2_{11}-2_{02}\right)_{(5 / 2-5 / 2)}$ in $\mathrm{G} 15 \mathrm{v} 2.779$. Right panel: from top to bottom are the spectra dominated by lines of $\mathrm{H}_{2} \mathrm{O}^{+}\left(2_{11}-2_{02}\right)_{(5 / 2-5 / 2)}, \mathrm{H}_{2} \mathrm{O}^{+}\left(2_{02}-1_{11}\right)_{(3 / 2-3 / 2)}$ and $\mathrm{H}_{2}^{18} \mathrm{O}\left(2_{11}-2_{02}\right)$, respectively, displayed as the filled yellow histograms. The reference frequency is the frequency of each of these lines. Weaker $\mathrm{H}_{2} \mathrm{O}^{+}\left(2_{02}-1_{11}\right)_{(3 / 2-3 / 2)}$ and $\mathrm{H}_{2} \mathrm{O}^{+}\left(2_{11}-2_{02}\right)_{(5 / 2-3 / 2)}$ components are indicated by additional grey vertical dashed lines. The superposed blue dashed histograms represent the spectra of para- $\mathrm{H}_{2} \mathrm{O}\left(2_{11}-2_{02}\right)$ in NCv1.143 centred at each line frequency. The red curve represents the Gaussian fitting to the spectra, and the green dashed curves are the decomposed Gaussian profiles for each fine structure line. The violet error bar indicates the $\pm 1 \sigma$ uncertainties of the spectrum.

flux densities of the two fine structure lines of $\mathrm{H}_{2} \mathrm{O}^{+}\left(2_{11}-2_{02}\right)$ are $1.6 \pm 0.5$ and $0.3 \pm 0.4 \mathrm{Jy} / \mathrm{km} \mathrm{s}^{-1}$, while they are $1.6 \pm 0.4$ and $0.2 \pm 0.5 \mathrm{Jy} / \mathrm{km} \mathrm{s}^{-1}$ for the two fine structure lines of $\mathrm{H}_{2} \mathrm{O}^{+}\left(2_{02}-1_{11}\right)$ (Table 8 ). We should note that these fitting results have much larger uncertainties due to the blending. Nevertheless, they are consistent with the earlier fitting results without de-blending. The similarity of the velocity-integrated flux densities between the $\mathrm{H}_{2} \mathrm{O}^{+}\left(2_{02}-1_{11}\right)$ and $\mathrm{H}_{2} \mathrm{O}^{+}\left(2_{11}-2_{02}\right)$ lines is in good agreement with the regime of far-infrared pumping as submm $\mathrm{H}_{2} \mathrm{O}$ (González-Alfonso et al. 2013). As a first approximation, if these $\mathrm{H}_{2} \mathrm{O}^{+}$lines are optically thin and we ignore the additional pumping from ortho- $\mathrm{H}_{2} \mathrm{O}^{+} 2_{02}$ to ortho- $\mathrm{H}_{2} \mathrm{O}^{+} J=3$ energy levels, the statistical equilibrium applied to energy level $2025 / 2$ implies that all population arriving per second at $2_{025 / 2}$ should be equal to all population leaving the level per second.

After subtracting the Gaussian profiles of all the $\mathrm{H}_{2} \mathrm{O}^{+}$ lines in the spectrum, we find a $3 \sigma$ residual in terms of the velocity-integrated flux density around $745.3 \mathrm{GHz}(I=0.6 \pm$ $0.2 \mathrm{Jy} \mathrm{km} \mathrm{s}^{-1}$, see Fig. 6). This could be a tentative detection of the $\mathrm{H}_{2}^{18} \mathrm{O}\left(2_{11}-2_{02}\right)$ line at $745.320 \mathrm{GHz}$. The velocity-integrated flux density ratio of $\mathrm{H}_{2}^{18} \mathrm{O}\left(2_{11}-2_{02}\right)$ over $\mathrm{H}_{2} \mathrm{O}\left(2_{11}-2_{02}\right)$ in $\mathrm{NCv} 1.143$ would hence be $\sim 0.1$. If this tentative detection was confirmed, it would show that ALMA could easily study such lines. But sophisticated models will be needed to infer isotope ratios.

The spectrum of the $\mathrm{H}_{2} \mathrm{O}\left(2_{11}-2_{02}\right)$ line in $\mathrm{G} 09 \mathrm{v} 1.97$ covers both the two main $\mathrm{H}_{2} \mathrm{O}^{+}$fine structure lines (Fig. 6). However, due to the limited sensitivity, we have only tentatively detected the $\mathrm{H}_{2} \mathrm{O}^{+}\left(2_{02}-1_{11}\right)_{(5 / 2-3 / 2)}$ line just above $3 \sigma$ (neglecting the minor contribution from $\left.\mathrm{H}_{2} \mathrm{O}^{+}\left(2_{11}-2_{02}\right)_{(5 / 2-3 / 2)}\right)$, and the velocity-integrated flux density is $1.4 \pm 0.4 \mathrm{Jy} \mathrm{km} \mathrm{s}^{-1}$ using a single Gaussian fit. We did not perform any line de-blending for this source considering the data quality. The $\mathrm{H}_{2} \mathrm{O}^{+}$line profile is in good agreement with that of the $\mathrm{H}_{2} \mathrm{O}$ (blue dashed histogram 
Table 8. Observed ortho- $\mathrm{H}_{2} \mathrm{O}^{+}$fine structure line parameters of the high-redshift $H$-ATLAS lensed HyLIRGs.

\begin{tabular}{lcrrr}
\hline \hline Source & $\mathrm{H}_{2} \mathrm{O}^{+}$transition & $\begin{array}{r}v_{\text {rest }} \\
(\mathrm{GHz})\end{array}$ & $\begin{array}{r}v_{\text {line }} \\
(\mathrm{GHz})\end{array}$ & $\begin{array}{r}\mathrm{I}_{\mathrm{H}_{2} \mathrm{O}^{+}} \\
\left(\mathrm{Jy} \mathrm{km} \mathrm{s}^{-1}\right)\end{array}$ \\
\hline $\mathrm{NCv1.143}$ & $2_{11-2}-2_{02(5 / 2-5 / 2)}$ & 746.5 & 163.53 & $1.6 \pm 0.5$ \\
& $2_{02}-1_{11(3 / 2-3 / 2)}$ & 746.3 & 163.48 & $0.2 \pm 0.5$ \\
& $2_{11}-2_{02}(5 / 2-3 / 2)$ & 742.3 & 162.61 & $0.3 \pm 0.4$ \\
& $2_{02}-1_{11(5 / 2-3 / 2)}$ & 742.1 & 162.56 & $1.6 \pm 0.4$ \\
$\mathrm{G} 09 \mathrm{v} 1.97$ & $2_{02}-1_{11(5 / 2-3 / 2)}$ & 742.1 & 160.14 & $1.4 \pm 0.4$ \\
$\mathrm{G} 15 \mathrm{v} 2.779$ & $2_{11}-2_{02(5 / 2-5 / 2)}$ & 746.5 & 142.35 & $1.2 \pm 0.3$ \\
\hline
\end{tabular}

Notes. The $\mathrm{H}_{2} \mathrm{O}^{+}\left(2_{02}-1_{11}\right)_{(5 / 2-3 / 2)}$ line in $\mathrm{G} 09 \mathrm{v} 1.97$ is blended by $\left(2_{11}-2_{02}\right)_{(5 / 2-3 / 2)}$, and $\mathrm{H}_{2} \mathrm{O}^{+}\left(2_{11}-2_{02}\right)_{(5 / 2-5 / 2)}$ line in G15v2.779 is blended by $\left(2_{02}-1_{11}\right)_{(3 / 2-3 / 2)}$. However, the contribution from the latter in each case is small, likely less than $20 \%$ as shown in the case of the $\mathrm{H}_{2} \mathrm{O}^{+}$lines in $\mathrm{NCv} 1.143$. Note that the quoted uncertainties do not include the missing parts of the spectra cut by the limited observed bandwidth (Fig. 6).

in Fig. 7). The velocity-integrated flux density of the undetected $\mathrm{H}_{2} \mathrm{O}^{+}\left(2_{11}-2_{02}\right)_{(5 / 2-5 / 2)}$ line could also be close to this value as discussed in the case of NCv1.143, yet somewhat lower and not detected in this source. More sensitive observation should be conducted to further derive robust line parameters.

We have also tentatively detected the $\mathrm{H}_{2} \mathrm{O}^{+}\left(2_{11}-2_{02}\right)_{(5 / 2-5 / 2)}$ line in G15v2.779 ( $S / N \sim 4$ by neglecting the minor contribution from the $\mathrm{H}_{2} \mathrm{O}^{+}\left(2_{02}-1_{11}\right)_{(3 / 2-3 / 2)}$ line $)$. The line profile is in good agreement with that of $\mathrm{H}_{2} \mathrm{O}\left(2_{11}-2_{02}\right)$ (blue dashed histogram in Fig. 6). The velocity-integrated flux density derived from a double-peak Gaussian fit is $1.2 \pm 0.3 \mathrm{Jy} \mathrm{km} \mathrm{s}^{-1}$ (we did not perform any line de-blending for the $\mathrm{H}_{2} \mathrm{O}^{+}$doublet considering the spectral noise level). There could be a minor contribution from the $\mathrm{H}_{2} \mathrm{O}^{+}\left(2_{02}-1_{11}\right)_{(3 / 2-3 / 2)}$ line to the velocity-integrated flux density. However, such a contribution is likely to be negligible as in the case of NCv1.143. The contribution is also within the uncertainty of the velocity-integrated flux density. Nevertheless, the position of $\mathrm{H}_{2} \mathrm{O}^{+}$has a small blue-shift compared with $\mathrm{H}_{2} \mathrm{O}$, but note that the blue part of the line is cut by the limited observed bandwidth (yellow histogram).

After including the local detections of $\mathrm{H}_{2} \mathrm{O}^{+}$lines from Y13 (Table B.1), we find a tight linear correlation between the luminosity of $\mathrm{H}_{2} \mathrm{O}$ and the two main lines of $\mathrm{H}_{2} \mathrm{O}^{+}$(slopes equal to $1.03 \pm 0.06$ and $0.91 \pm 0.07$, see Fig. 7). However, one should keep in mind that, because the local measurement done by Herschel SPIRE/FTS (Naylor et al. 2010) has rather low spectral resolution, neither $\mathrm{H}_{2} \mathrm{O}^{+}\left(2_{11}-2_{02}\right)(5 / 2-3 / 2)$ and $\mathrm{H}_{2} \mathrm{O}^{+}\left(2_{02}-1_{11}\right)_{(5 / 2-3 / 2)}$, nor $\mathrm{H}_{2} \mathrm{O}^{+}\left(2_{11}-2_{02}\right)_{(5 / 2-5 / 2)}$ and $\mathrm{H}_{2} \mathrm{O}^{+}\left(2_{02}-1_{11}\right)_{(3 / 2-3 / 2)}$ can be spectroscopically resolved. In the correlation plot (Fig. 7) and Table B.1, we use the total luminosity from the $742 \mathrm{GHz}$ and $746 \mathrm{GHz}$ lines, by assuming the contribution from $\mathrm{H}_{2} \mathrm{O}^{+}\left(2_{11}-2_{02}\right)_{(5 / 2-3 / 2)}$ and $\mathrm{H}_{2} \mathrm{O}^{+}\left(2_{02}-1_{11}\right)_{(3 / 2-3 / 2)}$ to the velocity-integrated flux density of the line at $742 \mathrm{GHz}$ and $746 \mathrm{GHz}$ is small $(\sim 18 \%)$ and does not vary significantly between different sources. Hence, the velocity-integrated flux density ratio between each of the two dominant $\mathrm{H}_{2} \mathrm{O}^{+}$fine structure lines and $\mathrm{H}_{2} \mathrm{O}$ in $\mathrm{NCv} 1.143, \mathrm{G} 15 \mathrm{v} 2.779$ and G09v1.97 is $\sim 0.3$ (uncertainties are less than $30 \%$ ), which is consistent with local galaxies as shown in the figure. This ratio is much larger than the abundance ratio of $\mathrm{H}_{2} \mathrm{O}^{+} / \mathrm{H}_{2} \mathrm{O} \sim 0.05$ found in Arp 220, an analogue of high-redshift ULIRGs (Rangwala et al. 2011).

As discussed above, the AGN contribution to the excitation of the submm lines of most of our sources appears to be minor. Thus, the formation of $\mathrm{H}_{2} \mathrm{O}^{+}$is likely dominated by cosmic-ray ionization, rather than X-ray ionization. Given the

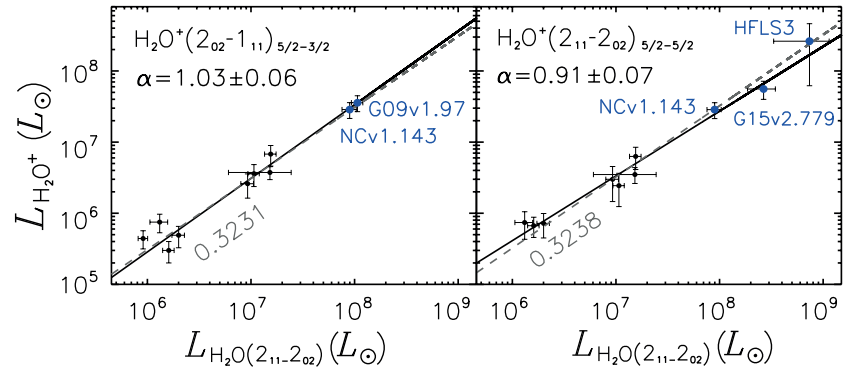

Fig. 7. Correlation between the luminosity of $J=2$ ortho- $\mathrm{H}_{2} \mathrm{O}^{+}$and para- $\mathrm{H}_{2} \mathrm{O}\left(2_{11}-2_{02}\right)$. The fitted function is $L_{\mathrm{H}_{2} \mathrm{O}^{+}} \propto L_{\mathrm{H}_{2} \mathrm{O}}{ }^{\alpha}$. We found a very good correlation between $L_{\mathrm{H}_{2} \mathrm{O}^{+}}$and $L_{\mathrm{H}_{2} \mathrm{O}}$ with a slope close to one. Black points are from the local ULIRGs as listed in Table B.1. Dark blue ones are high-redshift starbursts from this work. Black solid lines indicate the $\chi^{2}$ fitting results while the grey dashed lines and the grey annotations represent the average ratio between the $L_{\mathrm{H}_{2} \mathrm{O}^{+}}$and $L_{\mathrm{H}_{2} \mathrm{O}}$.

average luminosity ratio of $\mathrm{H}_{2} \mathrm{O}^{+} / \mathrm{H}_{2} \mathrm{O} \sim 0.3 \pm 0.1$ shown in Fig. 7, Meijerink et al. (2011) suggest a cosmic-ray ionization rate of $10^{-14}-10^{-13} \mathrm{~s}^{-1}$. Such high cosmic-ray ionization rates drive the ambient ionization degree of the ISM to $10^{-3}-10^{-2}$, rather than the canonical $10^{-4}$. Therefore, in the gas phase, an ion-neutral route likely dominates the formation of $\mathrm{H}_{2} \mathrm{O}$. However, $\mathrm{H}_{2} \mathrm{O}$ can also be enriched through the water-ice sublimation that releases $\mathrm{H}_{2} \mathrm{O}$ into the gas-phase ISM. As the upper part, $\sim 90 \mathrm{~K}$, of the possible range for $T_{\text {warm }}$ is close to the sublimation temperature of water ice. Hence, the high $\mathrm{H}_{2} \mathrm{O}$ abundance $\left(N_{\mathrm{H}_{2} \mathrm{O}} \gtrsim 0.3 \times 10^{17} \mathrm{~cm}^{-2}\right.$, see Sect. 4.2) observed is likely to be the result of ion chemistry dominated by high cosmic-ray ionization and/or perhaps water ice desorption. However, further observation of $\mathrm{H}_{2} \mathrm{O}^{+}$lines of different transitions and a larger sample is needed to constrain the contribution to $\mathrm{H}_{2} \mathrm{O}$ formation from neutral-neutral reactions dominated by shocks.

\section{Conclusions}

In this paper, we report a survey of submm $\mathrm{H}_{2} \mathrm{O}$ emission at redshift $z \sim 2-4$, by observing a higher excited ortho- $\mathrm{H}_{2} \mathrm{O}\left(3_{21}-3_{12}\right)$ in 6 sources and several complementary $J=2$ para- $\mathrm{H}_{2} \mathrm{O}$ emission lines in the warm dense cores of 11 high-redshift lensed extreme starburst galaxies (Hy/ULIRGs) discovered by $H$-ATLAS. So far, we have detected an $\mathrm{H}_{2} \mathrm{O}$ line in most of our observations of a total sample of 17 high-redshift lensed galaxies, in other words, we have detected both $J=2$ para- $\mathrm{H}_{2} \mathrm{O}$ and $J=3$ ortho- $\mathrm{H}_{2} \mathrm{O}$ lines in five, and in ten other sources only one $J=2$ para- $\mathrm{H}_{2} \mathrm{O}$ line. In these high-redshift $\mathrm{Hy} /$ ULIRGs, $\mathrm{H}_{2} \mathrm{O}$ is the second strongest molecular emitter after $\mathrm{CO}$ within the submm band, as in local ULIRGs. The spatially integrated $\mathrm{H}_{2} \mathrm{O}$ emission lines have a velocity-integrated flux density ranging from 4 to $15 \mathrm{Jy} \mathrm{km} \mathrm{s}^{-1}$, which yields the apparent $\mathrm{H}_{2} \mathrm{O}$ emission luminosity, $\mu L_{\mathrm{H}_{2} \mathrm{O}}$ ranging from $\sim 6-22 \times 10^{8} L_{\odot}$. After correction for gravitation lensing magnification, we obtained the intrinsic $L_{\mathrm{H}_{2} \mathrm{O}}$ for para- $\mathrm{H}_{2} \mathrm{O}$ lines $22_{02}-1_{11}, 2_{11}-2_{02}$ and ortho- $\mathrm{H}_{2} \mathrm{O}\left(3_{21}-3_{12}\right)$. The luminosities of the three $\mathrm{H}_{2} \mathrm{O}$ lines increase with $L_{\mathrm{IR}}$ as $L_{\mathrm{H}_{2} \mathrm{O}} \propto L_{\mathrm{IR}}{ }^{1.1-1.2}$. This correlation indicates the importance of far-infrared pumping as a dominant mechanism of $\mathrm{H}_{2} \mathrm{O}$ excitation. Comparing with $J=3$ to $J=6 \mathrm{CO}$ lines, the linewidths between $\mathrm{H}_{2} \mathrm{O}$ and $\mathrm{CO}$ are similar, and the velocity-integrated flux densities of $\mathrm{H}_{2} \mathrm{O}$ and $\mathrm{CO}$ are comparable. The similarity in line profiles suggests that these two molecular species possibly trace similar intense star-forming regions.

Using the far-infrared pumping model, we have analysed the ratios between $J=2$ and $J=3 \mathrm{H}_{2} \mathrm{O}$ lines and $L_{\mathrm{H}_{2} \mathrm{O}} / L_{\mathrm{IR}}$ in 5 sources with both $J \mathrm{H}_{2} \mathrm{O}$ lines detected. We have derived the 
ranges of the warm dust temperature $\left(T_{\text {warm }}\right)$, the $\mathrm{H}_{2} \mathrm{O}$ column density per unit velocity interval $\left(N_{\mathrm{H}_{2} \mathrm{O}} / \Delta V\right)$ and the optical depth at $100 \mu \mathrm{m}\left(\tau_{100}\right)$. Although there are strong degeneracies, these modelling efforts confirm that, similar to those of local ULIRGs, these submm $\mathrm{H}_{2} \mathrm{O}$ emissions in high-redshift Hy/ULIRGs trace the warm dense gas that is tightly correlated with the massive star forming activity. While the values of $T_{\text {warm }}$ and $N_{\mathrm{H}_{2} \mathrm{O}}$ (by assuming that they have similar velocity dispersion $\Delta V$ ) are similar to the local ones, $\tau_{100}$ in the high-redshift Hy/ULIRGs is likely to be greater than 1 (optically thick), which is larger than $\tau_{100}=0.05-0.2$ found in the local infrared galaxies. However, we notice that the parameter space is still not well constrained in our sources through $\mathrm{H}_{2} \mathrm{O}$ excitation modelling. Due to the limited excitation levels of the detected $\mathrm{H}_{2} \mathrm{O}$ lines, we are only able to perform the modelling with pure far-infrared pumping.

The detection of relatively strong $\mathrm{H}_{2} \mathrm{O}^{+}$lines opens the possibility to help understanding the formation of such large amount of $\mathrm{H}_{2} \mathrm{O}$. In these high-redshift $\mathrm{Hy} /$ ULIRGs, the $\mathrm{H}_{2} \mathrm{O}$ formation is likely to be dominated by ion-neutral reactions powered by cosmic-ray-dominated regions. The velocity-integrated flux density ratio between $\mathrm{H}_{2} \mathrm{O}^{+}$and $\mathrm{H}_{2} \mathrm{O}\left(I_{\mathrm{H}_{2} \mathrm{O}^{+}} / I_{\mathrm{H}_{2} \mathrm{O}} \sim 0.3\right)$, is remarkably constant from low to high-redshift, reflecting similar conditions in $\mathrm{Hy} / \mathrm{ULIRG}$. However, more observations of $\mathrm{H}_{2} \mathrm{O}^{+}$ emission/absorption and also $\mathrm{OH}^{+}$lines are needed to further constrain the physical parameters of the cosmic-ray-dominated regions and the ionization rate in those regions.

We have demonstrated that the submm $\mathrm{H}_{2} \mathrm{O}$ emission lines are strong and easily detectable with NOEMA. Being a unique diagnostic, the $\mathrm{H}_{2} \mathrm{O}$ emission offers us a new approach to constrain the physical conditions in the intense and heavily obscured star-forming regions dominated by far-infrared radiation at high-redshift. Follow-up observations of other gas tracers, for instance, $\mathrm{CO}, \mathrm{HCN}, \mathrm{H}_{2} \mathrm{O}^{+}$and $\mathrm{OH}^{+}$using the NOEMA, IRAM $30 \mathrm{~m}$ and JVLA will complement the $\mathrm{H}_{2} \mathrm{O}$ diagnostic of the structure of different components, dominant physical processes, star formation and chemistry in high-redshift Hy/ULIRGs.

With unprecedented spatial resolution and sensitivity, the image from the ALMA long baseline campaign observation of SDP 81 (also known as H-ATLAS J090311.6+003906, ALMA Partnership, Vlahakis et al. 2015; Dye et al. 2015; Rybak et al. 2015), shows the resolved structure of the dust, $\mathrm{CO}$ and $\mathrm{H}_{2} \mathrm{O}$ emission in the $z=3$ ULIRG. With careful reconstruction of the source plane images, ALMA will help to resolve the submm $\mathrm{H}_{2} \mathrm{O}$ emission in high-redshift galaxies into the scale of giant molecular clouds, and provide a fresh view of detailed physics and chemistry in the early Universe.

Acknowledgements. We thank our referee for the very detail comments and suggestions which have improved the paper. This work was based on observations carried out with the IRAM Interferometer NOEMA, supported by INSU/CNRS (France), MPG (Germany), and IGN (Spain). The authors are grateful to the IRAM staff for their support. C.Y. thanks Claudia Marka and Nicolas Billot for their help of the IRAM 30 m/EMIR observation. C.Y. also thanks Zhi-Yu Zhang and Iván Oteo for insightful discussions. C.Y., A.O. and Y.G. acknowledge support by NSFC grants \#11311130491, \#11420101002 and CAS Pilot B program \#XDB09000000. C.Y. and Y.G. also acknowledge support by NSFC grants \#11173059. C.Y., A.O., A.B. and Y.G. acknowledge support from the SinoFrench LIA-Origin joint exchange program. E.G.-A. is a Research Associate at the Harvard-Smithsonian Center for Astrophysics, and thanks the Spanish Ministerio de Economía y Competitividad for support under projects FIS2012-39162 C06-01 and ESP2015-65597-C4-1-R, and NASA grant ADAP NNX15AE56G. RJI acknowledges support from ERC in the form of the Advanced Investigator Programme, 321302, COSMICISM. US participants in $H$-ATLAS acknowledge support from NASA through a contract from JPL. Italian participants in $H$-ATLAS acknowledge a financial contribution from the agreement ASI-INAF I/009/10/0. SPIRE has been developed by a consortium of institutes led by Cardiff Univ. (UK) and including: Univ. Lethbridge (Canada); NAOC (China); CEA, LAM (France); IFSI, Univ. Padua (Italy); IAC (Spain); Stockholm
Observatory (Sweden); Imperial College London, RAL, UCL-MSSL, UKATC, Univ. Sussex (UK); and Caltech, JPL, NHSC, Univ. Colorado (USA). This development has been supported by national funding agencies: CSA (Canada); NAOC (China); CEA, CNES, CNRS (France); ASI (Italy); MCINN (Spain); SNSB (Sweden); STFC, UKSA (UK); and NASA (USA). C.Y. is supported by the China Scholarship Council grant (CSC No. 201404910443).

\section{References}

ALMA Partnership, Vlahakis, C., Hunter, T. R., Hodge, J. A., et al. 2015, ApJ, 808, L4

Becker, R. H., White, R. L., \& Helfand, D. J. 1995, ApJ, 450, 559

Bell, T. A., Viti, S., \& Williams, D. A. 2007, MNRAS, 378, 983

Bothwell, M. S., Aguirre, J. E., Chapman, S. C., et al. 2013, ApJ, 779, 67

Bournaud, F., Daddi, E., Weiß, A., et al. 2015, A\&A, 575, A56

Bradford, C. M., Bolatto, A. D., Maloney, P. R., et al. 2011, ApJ, 741, L37

Bussmann, R. S., Pérez-Fournon, I., Amber, S., et al. 2013, ApJ, 779, 25 (B13)

Calanog, J. A., Fu, H., Cooray, A., et al. 2014, ApJ, 797, 138

Casey, C. M., Narayanan, D., \& Cooray, A. 2014, Phys. Rep., 541, 45

Combes, F., Rex, M., Rawle, T. D., et al. 2012, A\&A, 538, L4

Condon, J. J. 1992, ARA\&A, 30, 575

Cooray, A., Calanog, J., Wardlow, J. L., et al. 2014, ApJ, 790, 40

Draine, B. T. 2003, ARA\&A, 41, 241

Dye, S., Furlanetto, C., Swinbank, A. M., et al. 2015, MNRAS, 452, 2258

Eales, S., Dunne, L., Clements, D., et al. 2010, PASP, 122, 499

Gao, Y., \& Solomon, P. M. 2004, ApJ, 606, 271

Gao, Y., Carilli, C. L., Solomon, P. M., \& Vanden Bout, P. A. 2007, ApJ, 660, L93

García-Burillo, S., Usero, A., Alonso-Herrero, A., et al. 2012, A\&A, 539, A8

Gérin, M., de Luca, M., Black, J., et al. 2010, A\&A, 518, L110

González-Alfonso, E., Smith, H. A., Fischer, J., \& Cernicharo, J. 2004, ApJ, 613, 247

González-Alfonso, E., Smith, H. A., Ashby, M. L. N., et al. 2008, ApJ, 675, 303

González-Alfonso, E., Fischer, J., Isaak, K., et al. 2010, A\&A, 518, L43

González-Alfonso, E., Fischer, J., Graciá-Carpio, J., et al. 2012, A\&A, 541, A4 González-Alfonso, E., Fischer, J., Bruderer, S., et al. 2013, A\&A, 550, A25

González-Alfonso, E., Fischer, J., Aalto, S., \& Falstad, N. 2014, A\&A, 567, A91 (G14)

Harris, A. I., Baker, A. J., Frayer, D. T., et al. 2012, ApJ, 752, 152

Ivison, R. J., Swinbank, A. M., Smail, I., et al. 2013, ApJ, 772, 137

Kamenetzky, J., Glenn, J., Rangwala, N., et al. 2012, ApJ, 753, 70

Kelly, B. C. 2007, ApJ, 665, 1489

Kennicutt, Jr., R. C. 1998, ARA\&A, 36, 189

Kessler, M. F., Steinz, J. A., Anderegg, M. E., et al. 1996, A\&A, 315, L27

Kirkpatrick, A., Pope, A., Sajina, A., et al. 2015, ApJ, 814, 9

Lis, D. C., Neufeld, D. A., Phillips, T. G., Gerin, M., \& Neri, R. 2011, ApJ, 738, L6

Liu, D., Gao, Y., Isaak, K., et al. 2015, ApJ, 810, L14

Lupu, R. E., Scott, K. S., Aguirre, J. E., et al. 2012, ApJ, 757, 135

Magdis, G. E., Daddi, E., Elbaz, D., et al. 2011, ApJ, 740, L15

Markwardt, C. B. 2009, in Astronomical Data Analysis Software and Systems XVIII, eds. D. A. Bohlender, D. Durand, \& P. Dowler, ASP Conf. Ser., 411, 251

Meijerink, R., \& Spaans, M. 2005, A\&A, 436, 397

Meijerink, R., Spaans, M., Loenen, A. F., \& van der Werf, P. P. 2011, A\&A, 525, A119

Meijerink, R., Kristensen, L. E., Weiß, A., et al. 2013, ApJ, 762, L16

Naylor, D. A., Baluteau, J.-P., Barlow, M. J., et al. 2010, in SPIE Conf. Ser., 7731,16

Negrello, M., Hopwood, R., De Zotti, G., et al. 2010, Science, 330, 800

Neufeld, D. A., Goicoechea, J. R., Sonnentrucker, P., et al. 2010, A\&A, 521, L10

Omont, A., Neri, R., Cox, P., et al. 2011, A\&A, 530, L3

Omont, A., Yang, C., Cox, P., et al. 2013, A\&A, 551, A115 (O13)

Pascale, E., Auld, R., Dariush, A., et al. 2011, MNRAS, 415, 911

Pellegrini, E. W., Smith, J. D., Wolfire, M. G., et al. 2013, ApJ, 779, L19

Pereira-Santaella, M., Spinoglio, L., Busquet, G., et al. 2013, ApJ, 768, 55

Pilbratt, G. L., Riedinger, J. R., Passvogel, T., et al. 2010, A\&A, 518, L1

Rangwala, N., Maloney, P. R., Glenn, J., et al. 2011, ApJ, 743, 94

Rawle, T. D., Egami, E., Bussmann, R. S., et al. 2014, ApJ, 783, 59

Riechers, D. A., Bradford, C. M., Clements, D. L., et al. 2013, Nature, 496, 329

Rybak, M., McKean, J. P., Vegetti, S., Andreani, P., \& White, S. D. M. 2015, MNRAS, 451, L40

San José-García, I., Mottram, J. C., van Dishoeck, E. F., et al. 2016, A\&A, 585, A103

Serjeant, S. 2012, MNRAS, 424, 2429

Solomon, P. M., Downes, D., \& Radford, S. J. E. 1992, ApJ, 387, L55

Spergel, D. N., Verde, L., Peiris, H. V., et al. 2003, ApJS, 148, 175 
Spinoglio, L., Pereira-Santaella, M., Busquet, G., et al. 2012, ApJ, 758, 108 van der Werf, P. P., Isaak, K. G., Meijerink, R., et al. 2010, A\&A, 518, L42 van der Werf, P. P., Berciano Alba, A., Spaans, M., et al. 2011, ApJ, 741, L38 van Dishoeck, E. F., Herbst, E., \& Neufeld, D. A. 2013, Chemical Reviews, 113, 9043

Vieira, J. D., Marrone, D. P., Chapman, S. C., et al. 2013, Nature, 495, 344

Weiß, A., Requena-Torres, M. A., Güsten, R., et al. 2010, A\&A, 521, L1

Weiß, A., De Breuck, C., Marrone, D. P., et al. 2013, ApJ, 767, 88

Wright, E. L., Eisenhardt, P. R. M., Mainzer, A. K., et al. 2010, AJ, 140 1868

Yang, C., Gao, Y., Omont, A., et al. 2013, ApJ, 771, L24 (Y13)

Yun, M. S., Reddy, N. A., \& Condon, J. J. 2001, ApJ, 554, 803

1 Purple Mountain Observatory/Key Lab of Radio Astronomy, Chinese Academy of Sciences, 210008 Nanjing, PR China e-mail: yangcht@pmo.ac . cn

2 Institut d'Astrophysique Spatiale, CNRS, Univ. Paris-Sud, Université Paris-Saclay, Bât. 121, 91405 Orsay Cedex, France

3 Graduate University of the Chinese Academy of Sciences, 19A Yuquan Road, Shijingshan District, 10049 Beijing, PR China

4 CNRS, UMR 7095, Institut d'Astrophysique de Paris, 75014 Paris, France

5 UPMC Univ. Paris 06, UMR 7095, Institut d'Astrophysique de Paris, 75014 Paris, France

6 Universidad de Alcalá, Departamento de Fisica y Matemáticas, Campus Universitario, 28871 Alcalá de Henares, Madrid, Spain

7 Institut de Radioastronomie Millimétrique (IRAM), 300 rue de la Piscine, 38406 Saint-Martin-d'Hères, France
${ }^{8}$ Leiden Observatory, Leiden University, PO Box 9513, 2300 RA Leiden, The Netherlands

9 Max Planck Institut für Radioastronomie, Auf dem Hgel 69, 53121 Bonn, Germany

10 Department of Earth and Space Sciences, Chalmers University of Technology, Onsala Space Observatory, 43992 Onsala, Sweden

11 Department of Physics and Astronomy, Rutgers, The State University of New Jersey, 136 Frelinghuysen Road, Piscataway, NJ 08854-8019, USA

12 Astronomy Department, Cornell University, 220 Space Sciences Building, Ithaca, NY 14853, USA

13 Department of Physics and Astronomy, University of California, Irvine, Irvine, CA 92697, USA

14 Joint ALMA Observatory, 3107 Alonso de Córdova, Vitacura, Santiago, Chile

15 Universitat Wien, Institut für Astrophysik, Türkenschanzstrasse 17, 1180 Wien, Austria

16 School of Physics and Astronomy, University of Nottingham, University Park, Nottingham NG7 2RD, UK

17 Institute for Astronomy, University of Edinburgh, Royal Observatory, Blackford Hill, Edinburgh, EH9 3HJ, UK

18 European Southern Observatory, Karl Schwarzschild Straße 2, 85748 Garching, Germany

19 Kapteyn Astronomical Institute, University of Groningen, 9700 AV Groningen, The Netherlands

20 School of Physics and Astronomy, Cardiff University, The Parade, Cardiff CF24 3AA, UK 


\section{Appendix A: Individual sources}

In the Appendix, we describe the observational results of each source, including the lensing model, the $\mathrm{H}_{2} \mathrm{O}$ spectrum, mapping of the $\mathrm{H}_{2} \mathrm{O}$ and continuum emission (by showing lowresolution NOEMA $\mathrm{H}_{2} \mathrm{O}$ and continuum images), the ratio between velocity-integrated flux densities of different $\mathrm{H}_{2} \mathrm{O}$ transitions, and the comparison between $\mathrm{H}_{2} \mathrm{O}$ and $\mathrm{CO}$ emission.

\section{A.1. G09v1.97 at $z=3.634$}

The galaxy G09v1.97 has the second largest redshift in our sample obtained by CARMA (Riechers et al., in prep.). In the SMA $880 \mu \mathrm{m}$ image (B13), similar to SDP81 (ALMA Partnership, Vlahakis et al. 2015; Dye et al. 2015), it displays a triple arc structure with an angular size scale of $\sim 2^{\prime \prime}$. However, there are two foreground deflectors at two different redshifts, making this complex mass distribution a very unusual case. B13 estimate a lensing amplification $\mu=6.9 \pm 0.6$.

We have observed both para- $\mathrm{H}_{2} \mathrm{O}\left(2_{11}-2_{02}\right)$ and ortho$\mathrm{H}_{2} \mathrm{O}\left(3_{21}-3_{12}\right)$ lines at $162 \mathrm{GHz}$ and $251 \mathrm{GHz}$, respectively. The source is clearly unresolved at $162 \mathrm{GHz}$, but marginally resolved at $251 \mathrm{GHz}$ as displayed in Fig. A.1. The ratio between the peak and the spatially integrated flux density of the continuum $\left(S_{v}(\mathrm{ct})^{\mathrm{pk}} / S_{v}(\mathrm{ct})\right)$ is $0.95 \pm 0.03$ and $0.60 \pm 0.01$ at $162 \mathrm{GHz}$ and $251 \mathrm{GHz}$, respectively. The $\mathrm{H}_{2} \mathrm{O}$ emission line peak to spatially integrated flux densiity ratio $\left(S_{\mathrm{H}_{2} \mathrm{O}}^{\mathrm{pk}} / S_{\mathrm{H}_{2} \mathrm{O}}\right)$ for $J=2$ and $J=3$ are $1.0 \pm 0.2$ and $0.5 \pm 0.2$, respectively. Therefore, the spatial concentrations of $\mathrm{H}_{2} \mathrm{O}$ and continuum image are in good agreement within the uncertainties.

Both the continuum and the $\mathrm{H}_{2} \mathrm{O}$ lines are well detected in this source. The two $\mathrm{H}_{2} \mathrm{O}$ lines are well fitted by single Gaussian profiles with similar linewidths $\left(257 \pm 27\right.$ and $234 \pm 34 \mathrm{~km} \mathrm{~s}^{-1}$, Fig. 2 and Table 4). The difference in linewidth $\left(23 \mathrm{~km} \mathrm{~s}^{-1}\right)$ is smaller than the errors of the linewidth. Therefore, there is no significant difference between the spectra of the two transitions. The ratio between $I_{\mathrm{H}_{2} \mathrm{O}\left(3_{21}-3_{12}\right)}$ and $I_{\mathrm{H}_{2} \mathrm{O}\left(2_{11}-2_{02}\right)}$ is $0.91 \pm 0.12$, which is the lowest of our five detected sources in both lines. However, this ratio remains consistent with the observations of local galaxies (Y13, and Fig. 4), by taking the uncertainty into account.

From our CO line observations we find a line FWHM of $\Delta V_{\mathrm{CO}}=224 \pm 32$ and $292 \pm 86 \mathrm{~km} \mathrm{~s}^{-1}$ for the $\mathrm{CO}(5-4)$ and $\mathrm{CO}(6-5)$ lines, respectively, which are within $1 \sigma$ to the $\mathrm{H}_{2} \mathrm{O}$ FWHMs. The observed ratio of $I_{\mathrm{H}_{2} \mathrm{O}} / I_{\mathrm{CO}}$ for both the $\mathrm{CO}(5-4)$ and $\mathrm{CO}(6-5)$ lines, is about 0.4 with less than $25 \%$ uncertainty.

We have tentatively detected an $\mathrm{H}_{2} \mathrm{O}^{+}$line in this source as well (see Sect. 5).

\section{A.2. $G 12 v 2.43$ at $z=3.127$}

The source is marginally resolved in the SMA $880 \mu \mathrm{m}$ image (Fig. 2 of B13), with a size $\sim 1.5^{\prime \prime}$, but there is no obvious strongly lensed structures such as multiple images. It is not yet possible to build a lensing model for this source because the search for a deflector by B13 has been unsuccessful.

Both para- $\mathrm{H}_{2} \mathrm{O}\left(2_{02}-1_{11}\right)$ and ortho- $\mathrm{H}_{2} \mathrm{O}\left(3_{21}-3_{12}\right)$ lines are well detected, as shown in Fig. A.1, and the source is unresolved, consistent with the SMA image. The ratios of $S_{v}(\mathrm{ct})^{\mathrm{pk}} / S_{v}(\mathrm{ct})$ for $239 \mathrm{GHz}$ and $282 \mathrm{GHz}$ are $0.71 \pm 0.02$ and $0.87 \pm 0.01$, respectively, while the $S_{\mathrm{H}_{2} \mathrm{O}}^{\mathrm{pk}} / S_{\mathrm{H}_{2} \mathrm{O}}$ for $\mathrm{H}_{2} \mathrm{O}\left(2_{02}-1_{11}\right)$ and $\mathrm{H}_{2} \mathrm{O}\left(3_{21}-3_{12}\right)$ are $0.6 \pm 0.2$ and $1.0 \pm 0.2$, respectively.
The two $\mathrm{H}_{2} \mathrm{O}$ lines are both well fitted by a single Gaussian profile. The FWHMs are $201 \pm 27$ and $221 \pm 20 \mathrm{~km} \mathrm{~s}^{-1}$ for para$\mathrm{H}_{2} \mathrm{O}\left(2_{02}-1_{11}\right)$ and ortho- $\mathrm{H}_{2} \mathrm{O}\left(3_{21}-3_{12}\right)$, respectively. The difference is within the $1 \sigma$ uncertainty.

The velocity-integrated flux density ratio of high-lying over

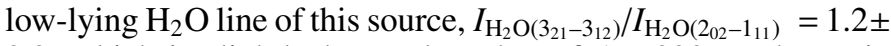
0.2 , which is slightly lower than that of Arp 220 as shown in Fig. 4. The linewidths of the $\mathrm{H}_{2} \mathrm{O}$ lines $(201 \pm 27$ and $221 \pm$ $20 \mathrm{~km} \mathrm{~s}^{-1}$ ) are the narrowest ones among our sources. The values are also very close to the $\mathrm{CO}(1-0)$ linewidth $\left(210 \pm 30 \mathrm{~km} \mathrm{~s}^{-1}\right.$, Harris et al. 2012). Their similarity indicates that there is not likely any strong differential lensing effect between the $\mathrm{CO}$ and $\mathrm{H}_{2} \mathrm{O}$ emissions in this case.

\section{A.3. $N C v 1.143$ at $z=3.565$}

Having a redshift of $z=3.565$ from CO observation by CARMA (Riechers et al., in prep.), this source is one of the brightest (at submm) in our sample. It is resolved by the SMA $880 \mu \mathrm{m}$ beam (B13) with a size $\sim 2^{\prime \prime}$, featured by two components at the northeast and southwest directions. With a single deflector, the lensing model estimates a magnification factor of $\mu=6.9 \pm 0.6$.

As displayed in Fig. A.1, both the lines and the continuum are very strong and well detected. The ratio $S_{v}(\mathrm{ct})^{\mathrm{pk}} / S_{v}(\mathrm{ct})=$ $0.86 \pm 0.02$ shows that the source is unresolved at $165 \mathrm{GHz}$ (for observing para- $\mathrm{H}_{2} \mathrm{O}$ line $2_{11}-2_{02}$ ). At $255 \mathrm{GHz}$, the ratios $S_{v}(\mathrm{ct})^{\mathrm{pk}} / S_{v}(\mathrm{ct})=0.55 \pm 0.01$ and $S_{\mathrm{H}_{2} \mathrm{O}}^{\mathrm{pk}} / S_{\mathrm{H}_{2} \mathrm{O}}=0.7 \pm 0.2$ indicate that the source is partially resolved, consistent with the SMA result.

Both the $\mathrm{H}_{2} \mathrm{O}\left(2_{02}-1_{11}\right)$ and $\mathrm{H}_{2} \mathrm{O}\left(3_{21}-3_{12}\right)$ lines can be fitted by single Gaussian profiles. The ratio of $I_{\mathrm{H}_{2} \mathrm{O}\left(3_{21}-3_{12}\right)} / I_{\mathrm{H}_{2} \mathrm{O}\left(2_{11}-2_{02}\right)}$ is $1.36 \pm 0.13$, close to the mean ratio found in the nearby starforming-dominated galaxies (Y13, and Fig. 4). The linewidths of $\mathrm{H}_{2} \mathrm{O}\left(2_{11}-2_{02}\right)$ and $\mathrm{H}_{2} \mathrm{O}\left(3_{21}-3_{12}\right)$ are $293 \pm 15$ and $233 \pm$ $22 \mathrm{~km} \mathrm{~s}^{-1}$, respectively. Although the former is larger, they are compatible within an uncertainty of $1.6 \sigma$. Also, the $\mathrm{H}_{2} \mathrm{O}$ linewidth agrees well with the $\mathrm{CO}(5-4)$ and $\mathrm{CO}(6-5)$ linewidths $\left(273 \pm 27\right.$ and $284 \pm 27 \mathrm{~km} \mathrm{~s}^{-1}$, see Table 3$)$. Therefore, the line ratios are unlikely to be affected by differential magnification. The observed ratio of $I_{\mathrm{H}_{2} \mathrm{O}} / I_{\mathrm{CO}}$ is $0.4-0.5$ and $0.6-0.7$ (uncertainties are within $13 \%$ ) for the $J=2$ para- $\mathrm{H}_{2} \mathrm{O}$ and $J=3$ ortho- $\mathrm{H}_{2} \mathrm{O}$, respectively.

We have also detected ortho- $\mathrm{H}_{2} \mathrm{O}^{+}\left(2_{11}-2_{02}\right)$ and ortho$\mathrm{H}_{2} \mathrm{O}^{+}\left(2_{02}-1_{11}\right)$ fine structure lines together with para$\mathrm{H}_{2} \mathrm{O}\left(2_{11}-2_{02}\right)$ in this source. The further discussion of the $\mathrm{H}_{2} \mathrm{O}^{+}$spectra and its interpretation are given in Sect. 5 .

\section{A.4. NAv1.195 at $z=2.951$}

As quoted in B13, the redshift of this source was first obtained by the CO observation (Harris et al., in prep.). Its SMA image shows a typical lensed feature with two components separated by $\sim 2^{\prime \prime}$ along the northwest and southeast direction. The lensing model suggests a modest magnification factor $\mu=4.1 \pm 0.3$.

We have robust detections of $\mathrm{H}_{2} \mathrm{O}\left(2_{02}-1_{11}\right)$ and the continuum emission at $250 \mathrm{GHz}$ and $293 \mathrm{GHz}$ (Fig. A.1). However, the $\mathrm{H}_{2} \mathrm{O}\left(3_{21}-3_{12}\right)$ line isat odds with the other five sources. Therefore, we only show the image of the dust continuum emission at this frequency in Fig. A.1. The source is clearly resolved into two components in the three images, and the northwest component is about 4 times stronger than the southeast one in the continuum images, in agreement with the SMA image (B13). For the continuum at $250 \mathrm{GHz}$, the peak to total integrated flux density 

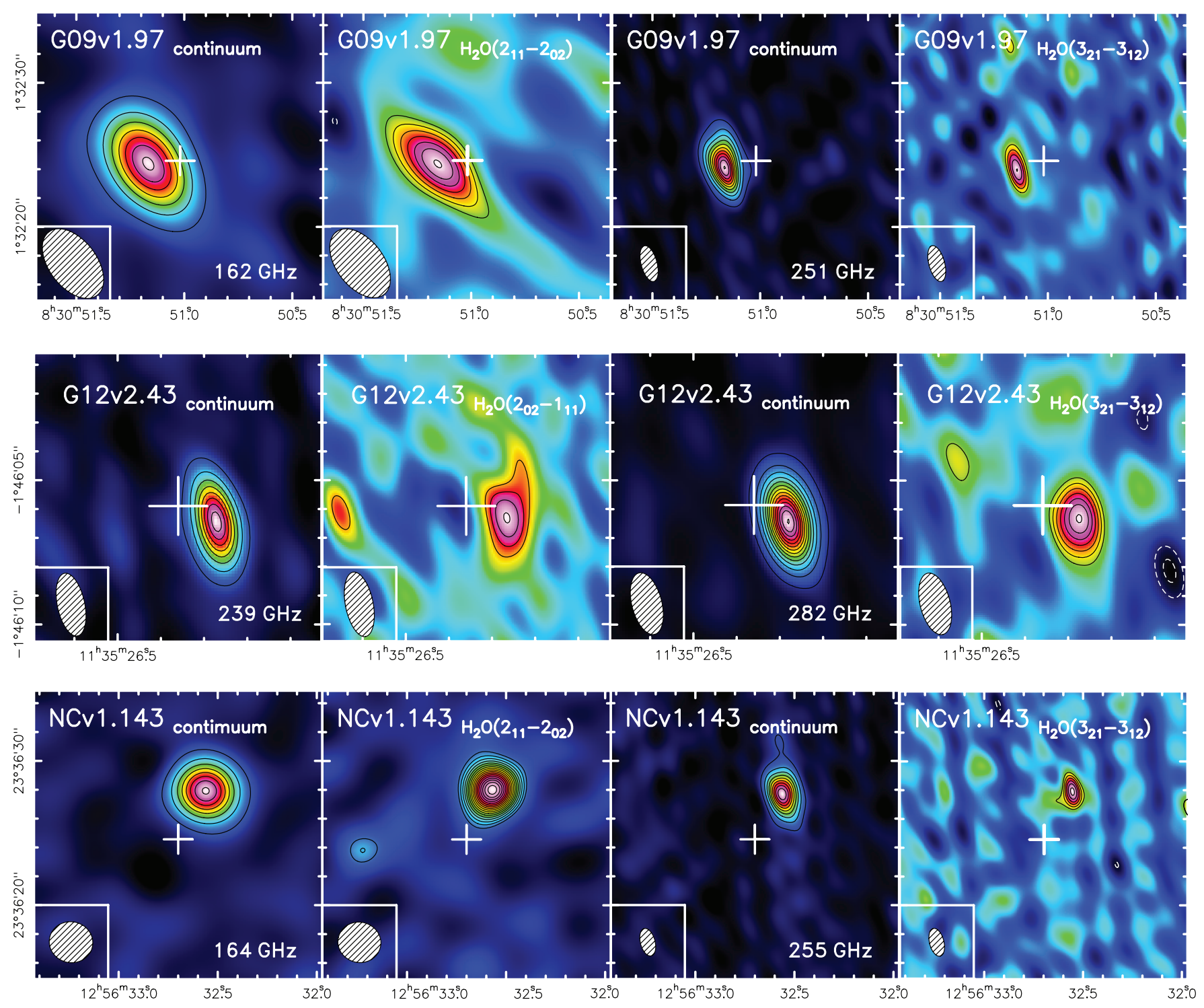

Fig. A.1. Mapping of the $\mathrm{H}_{2} \mathrm{O}$ emission lines and the corresponding continuum emission (frequencies have been shown accordingly in the white text) in the sources with both para $J=2$ and ortho $J=3 \mathrm{H}_{2} \mathrm{O}$ lines observed. The contours of the continuum emission start from $6 \sigma$ in step of $10 \sigma$, and the contours of the $\mathrm{H}_{2} \mathrm{O}$ emission start from $3 \sigma$ in step of $1 \sigma$. Asymmetric negative contours are shown in white dashed lines. For each observation, the $1 \sigma$ contours for the continuum $\left(\mathrm{mJy}\right.$ beam ${ }^{-1}$ ) and the $\mathrm{H}_{2} \mathrm{O}$ emission line $\left(\mathrm{Jy} \mathrm{km} \mathrm{s}^{-1} \mathrm{beam}^{-1}\right)$ are as follows: G09v1.97 $\mathrm{H}_{2} \mathrm{O}\left(2_{11}-2_{02}\right)(0.17 / 0.57), \mathrm{G} 09 \mathrm{v} 1.97 \mathrm{H}_{2} \mathrm{O}\left(3_{21}-3_{12}\right)(0.25 / 0.38), \mathrm{G} 12 \mathrm{v} 2.43 \mathrm{H}_{2} \mathrm{O}\left(2_{02}-1_{11}\right)(0.29 / 0.48), \mathrm{G} 12 \mathrm{v} 2.43 \mathrm{H}_{2} \mathrm{O}\left(3_{21}-3_{12}\right)$ (0.30/0.53), NCv1.143 $\mathrm{H}_{2} \mathrm{O}\left(2_{11}-2_{02}\right)(0.16 / 0.36)$ and $\mathrm{NCv} 1.143 \mathrm{H}_{2} \mathrm{O}\left(3_{21}-3_{12}\right)(0.42 / 0.72)$.

ratio $S_{v}(\mathrm{ct})^{\mathrm{pk}} / S_{v}(\mathrm{ct})=0.54 \pm 0.02$, and for $\mathrm{H}_{2} \mathrm{O}\left(2_{02}-1_{11}\right)$, the ratio $S_{\mathrm{H}_{2} \mathrm{O}}^{\mathrm{pk}} / S_{\mathrm{H}_{2} \mathrm{O}}$ equals to $0.6 \pm 0.3$. Therefore, the spatial distributions of dust and the $\mathrm{H}_{2} \mathrm{O}$ emission are likely to be similar in this source. In the observation at $293 \mathrm{GHz}, S_{v}(\mathrm{ct})^{\mathrm{pk}} / S_{v}(\mathrm{ct})=$ $0.42 \pm 0.01$, due to a smaller synthesis beam (Table 1 ).

Figure 2 shows the spectra corresponding to the two observations of NAv1.195. The $\mathrm{H}_{2} \mathrm{O}\left(2_{02}-1_{11}\right)$ line can be fitted by a single Gaussian profile, with a linewidth equal to $328 \pm 51 \mathrm{~km} \mathrm{~s}^{-1}$. We have not detected the higher excitation $\mathrm{H}_{2} \mathrm{O}\left(3_{21}-3_{12}\right)$ line as mentioned above. By assuming the same linewidth as the lower- $J \mathrm{H}_{2} \mathrm{O}$ line, we can infer a $2 \sigma$ detection limit of $2.56 \mathrm{Jy} \mathrm{km} \mathrm{s}^{-1}$. This yields a flux ratio of $\mathrm{H}_{2} \mathrm{O}\left(3_{21}-3_{12}\right) / \mathrm{H}_{2} \mathrm{O}\left(2_{02}-1_{11}\right) \lesssim 0.6$. This value is significantly lower than that in the five other sources where it ranges from 0.75 to 1.60 (errors are within $25 \%$ ), but it remains close to the lowest values measured in local galaxies (Y13) as shown in Table 6 and Fig. 4. This low ratio of $\mathrm{H}_{2} \mathrm{O}$ lines probably originates from different excitation conditions, especially for the far-infrared radiation field, since the line $\mathrm{H}_{2} \mathrm{O}\left(3_{21}-3_{12}\right)$ is mainly populated through far-infrared pumping via absorbing $75 \mu \mathrm{m}$ photons (see Sect. 5). The $\mathrm{CO}(5-4)$ line of the source has a linewidth of $281 \pm 16 \mathrm{~km} \mathrm{~s}^{-1}$, which is comparable with the $\mathrm{H}_{2} \mathrm{O}$ line profile. The observed ratio of $I_{\mathrm{H}_{2} \mathrm{O}} / I_{\mathrm{CO}}(\mathrm{CO}(5-4))$ is $\leq 0.4$.

\section{A.5. NAv1.177 at $z=2.778$}

NOEMA observation of the CO line in this source gives a redshift of $z=2.778$ (Krips et al., in prep.). The SMA $880 \mu \mathrm{m}$ image shows a compact structure with two peaks $\sim 1^{\prime \prime}$ away along the eastwest direction, and the western component is the dominant one (Fig. 2 of B13). However, due to the absence of deflector in the foreground optical image from SDSS and lack of the deep optical and near-infrared images, the lensing properties are still unknown (B13). 

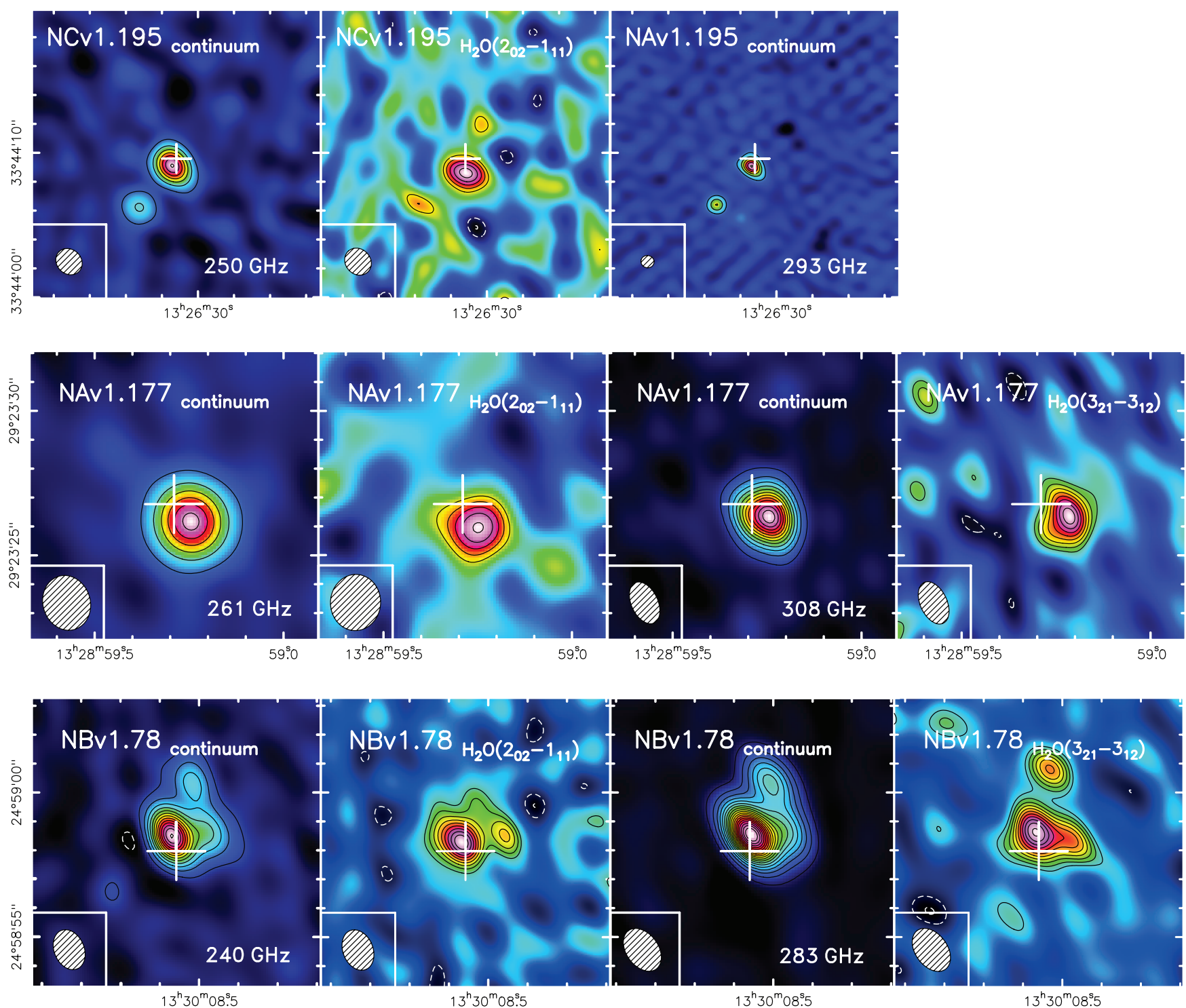

Fig. A.2. (See Fig. A.1 caption.) For each observation, the $1 \sigma$ contour for the continuum (mJybeam ${ }^{-1}$ ) and the $\mathrm{H}_{2} \mathrm{O}$ emission line $\left(\mathrm{Jy} \mathrm{km} \mathrm{s}^{-1}\right.$ beam $^{-1}$ ) are as follows: $\mathrm{NCv} 1.195 \mathrm{H}_{2} \mathrm{O}\left(2_{02}-1_{11}\right)(0.34 / 0.51)$, NCv1.195 $\mathrm{H}_{2} \mathrm{O}\left(3_{21}-3_{12}\right)(0.48 /-)$, NAv1.177 $\mathrm{H}_{2} \mathrm{O}\left(2_{02}-1_{11}\right)(0.58 / 0.65)$, $\mathrm{NAv} 1.177 \mathrm{H}_{2} \mathrm{O}\left(3_{21}-3_{12}\right)(0.38 / 0.58)$, NBv1.78 $\mathrm{H}_{2} \mathrm{O}\left(2_{02}-1_{11}\right)(0.28 / 0.30)$, $\mathrm{NBv} 1.78 \mathrm{H}_{2} \mathrm{O}\left(3_{21}-3_{12}\right)(0.21 / 0.29)$.

As displayed in Fig. A.1, both the lines of $\mathrm{H}_{2} \mathrm{O}\left(2_{02}-1_{11}\right)$ and $\mathrm{H}_{2} \mathrm{O}\left(3_{21}-3_{12}\right)$ and the corresponding dust continuum are well detected. The ratios $S_{v}(\mathrm{ct})^{\mathrm{pk}} / S_{v}(\mathrm{ct})$ are $0.75 \pm 0.02$ and $0.62 \pm 0.01$ for observation at $261 \mathrm{GHz}$ and $308 \mathrm{GHz}$, respectively. One should notice that the direction of the synthesised beam is perpendicular to the alignment of the two components in the image, thus the source is marginally resolved in the $\mathrm{H}_{2} \mathrm{O}\left(2_{02}-1_{11}\right)$ and the corresponding dust continuum images. For the $\mathrm{H}_{2} \mathrm{O}\left(3_{21}-3_{12}\right)$ observation at higher frequency, we can see the partially resolved feature. The peak to total flux ratios of $\mathrm{H}_{2} \mathrm{O}$ are $S_{\mathrm{H}_{2} \mathrm{O}}^{\mathrm{pk}} / S_{\mathrm{H}_{2} \mathrm{O}} \sim 0.8 \pm 0.3$ and $0.6 \pm 0.1$ for the $\mathrm{H}_{2} \mathrm{O}\left(2_{02}-1_{11}\right)$ and $\mathrm{H}_{2} \mathrm{O}\left(3_{21}-3_{12}\right)$ lines, respectively, indicating similar spatial distribution compared with the dust emission.

The $\mathrm{H}_{2} \mathrm{O}$ spectra displayed in Fig. 2 show single Gaussian profiles with $F W H M=241 \pm 41$ and $272 \pm 24 \mathrm{~km} \mathrm{~s}^{-1}$ (Table 4). The profiles of the two $\mathrm{H}_{2} \mathrm{O}$ lines are similar within the uncertainties. The line ratio, $I_{\mathrm{H}_{2} \mathrm{O}\left(3_{21}-3_{12}\right)} / I_{\mathrm{H}_{2} \mathrm{O}\left(2_{02}-1_{11}\right)}=1.34 \pm 0.24$. This value is close to that found in Arp 220 and it is the largest ratio in our sample. We have also detected the $\mathrm{CO}(3-2)$ and $\mathrm{CO}(5-4)$ lines using the IRAM $30 \mathrm{~m}$ telescope in this source (Table 3), the linewidth of $\mathrm{CO}$ is around $230 \pm 16 \mathrm{~km} \mathrm{~s}^{-1}$ which is similar to the width of the detected $\mathrm{H}_{2} \mathrm{O}$ lines. The ratio of $I_{\mathrm{H}_{2} \mathrm{O}} / I_{\mathrm{CO}}$ is from 0.5 to 1.1 with less than $20 \%$ uncertainties.

\section{A.6. $N B v 1.78$ at $z=3.111$}

The CO redshift of NBv1.78 was obtained by Riechers et al. (in prep.) via CARMA, $z=3.111$, and the data of the $\mathrm{H}_{2} \mathrm{O}\left(2_{02}-1_{11}\right)$ line were obtained by $\mathrm{O} 13$. The source is resolved in the SMA $880 \mu \mathrm{m}$ image (B13) with a somewhat complex morphology, and the size is $\sim 2.5^{\prime \prime}$. There are three main components in the image. The two strong components located at northwest and southeast direction of the image, and the weakest component close to the southeast. The derived lensing magnification is $\mu=13.5 \pm 1.5$, which is the second largest among our sample. In the near-infrared images, the source has a similar threecomponent Einstein ring-like structure with a slightly smaller magnification $\mu=10.8_{-0.2}^{+0.3}$ (Calanog et al. 2014).

Our NOEMA images of both continuums and $\mathrm{H}_{2} \mathrm{O}$ lines as shown in Fig. A.1 are consistent with the SMA $880 \mu \mathrm{m}$ image. 

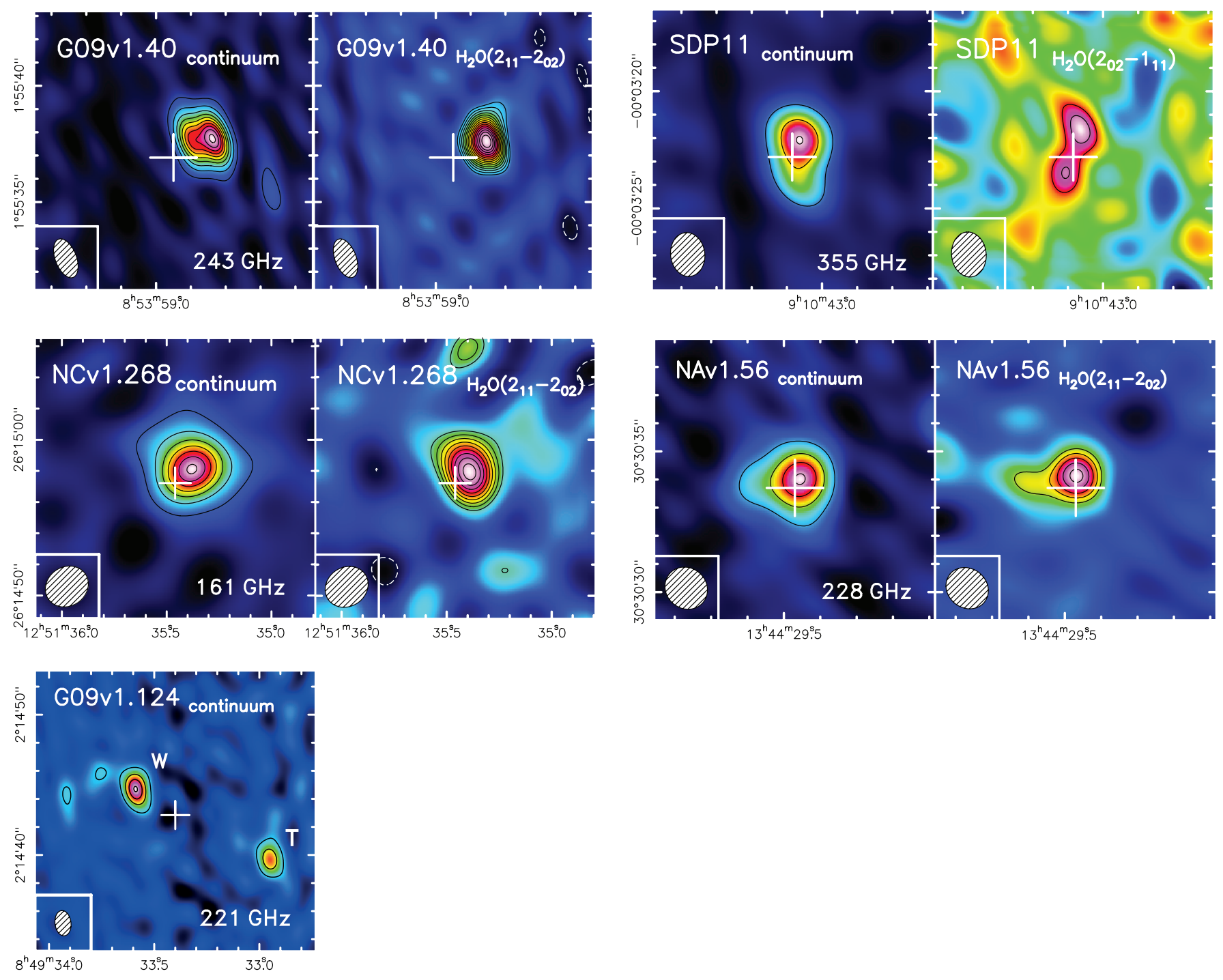

Fig. A.3. (See Fig. A.1 caption.) For each observation, the $1 \sigma$ contour for the continuum (mJybeam ${ }^{-1}$ ) and the $\mathrm{H}_{2} \mathrm{O}$ emission line $\left(\mathrm{Jy} \mathrm{km} \mathrm{s}^{-1}\right.$ beam $^{-1}$ ) are as follows: G09v1.124 $\mathrm{H}_{2} \mathrm{O}\left(2_{11}-2_{02}\right)(0.17 /-)$, G09v1.40 $\mathrm{H}_{2} \mathrm{O}\left(2_{11}-2_{02}\right)(0.19 / 0.32)$, SDP11 $\mathrm{H}_{2} \mathrm{O}\left(2_{02}-1_{11}\right)(1.30 / 1.04)$, $\mathrm{NCv} 1.268 \mathrm{H}_{2} \mathrm{O}\left(2_{11}-2_{02}\right)(0.13 / 0.39)$ and $\mathrm{NAv} 1.56 \mathrm{H}_{2} \mathrm{O}\left(2_{11}-2_{02}\right)(0.53 / 1.02)$.

The images are resolved into two main parts, while the southern component is extended along the western side. The continuum and $\mathrm{H}_{2} \mathrm{O}$ line images have fairly high $\mathrm{S} / \mathrm{N}$. From the observation of $\mathrm{H}_{2} \mathrm{O}\left(2_{02}-1_{11}\right)$ at $241 \mathrm{GHz}(\mathrm{O} 13$, note that this observation was performed at higher resolution with a $1.4^{\prime \prime} \times 1.0^{\prime \prime}$ beam), the values of $S_{v}(\mathrm{ct})^{\mathrm{pk}} / S_{v}(\mathrm{ct})$ and $S_{\mathrm{H}_{2} \mathrm{O}}^{\mathrm{pk}} / S_{\mathrm{H}_{2} \mathrm{O}}$ agree well, which are $0.42 \pm 0.01$ and $0.4 \pm 0.1$, respectively. The continuum image at $283 \mathrm{GHz}$ gives $S_{v}(\mathrm{ct})^{\mathrm{pk}} / S_{v}(\mathrm{ct})=0.69 \pm 0.01$, and the image of $\mathrm{H}_{2} \mathrm{O}\left(3_{21}-3_{12}\right)$ gives $S_{\mathrm{H}_{2} \mathrm{O}}^{\mathrm{pk}} / S_{\mathrm{H}_{2} \mathrm{O}}=0.8 \pm 0.1$. The similarity of the peak to spatially integrated flux density ratios suggest that the spatial distribution of $\mathrm{H}_{2} \mathrm{O}$ and submm dust continuum are likely to be consistent. Additionally, the images of $J=2$ and $J=3$ images are also consistent within the uncertainty.

NBv1.78 has a very broad linewidth compared with the other sources. As shown in Fig. 2, the linewidth of $\mathrm{H}_{2} \mathrm{O}\left(2_{02}-1_{11}\right)$ and $\mathrm{H}_{2} \mathrm{O}\left(3_{21}-3_{12}\right)$ are $510 \pm 90$ and $607 \pm 43 \mathrm{~km} \mathrm{~s}^{-1}$, respectively. The two lines have similar profiles. The source has a $I_{\mathrm{H}_{2} \mathrm{O}\left(3_{21}-3_{12}\right)} / I_{\mathrm{H}_{2} \mathrm{O}\left(2_{02}-1_{11}\right)}$ ratio equal to 1 , within the range of the local galaxies (Fig. 4). The $\mathrm{CO}(5-4)$ and $\mathrm{CO}(6-5)$ observations (Table 3 ) give linewidths of $614 \pm 53$ and $734 \pm 85 \mathrm{~km} \mathrm{~s}^{-1}$, which are wider than the $\mathrm{H}_{2} \mathrm{O}$ lines. The observed ratio of $I_{\mathrm{H}_{2} \mathrm{O}} / I_{\mathrm{CO}}$ is about 0.7 with less than $25 \%$ uncertainty for $J=2 \mathrm{H}_{2} \mathrm{O}$.

\section{A.7. $G 09 v 1.40$ at $z=2.093$}

A CO redshift of G09v1.40, $z=2.0894$ was obtained by $\mathrm{CSO} / \mathrm{Z}-\mathrm{Spec}$ (Lupu et al., in prep.). But, through our $\mathrm{H}_{2} \mathrm{O}$ observation, we find a redshift of $z=2.093$. Our value is consistent with the $\mathrm{CO}(3-2)$ observation by Riechers et al. (in prep.), and we have adopted this value. SMA observation of the $880 \mu \mathrm{m}$ dust continuum shows two close components with a separation of $\sim 1^{\prime \prime}$ along the east and west direction. The lensing model estimates $\mu=15.3 \pm 3.5$, which is the largest magnification in our sample. The Keck near-infrared image of the source suggests a magnification of $\mu=11.4_{-1.0}^{+0.9}$ for the stellar component (Calanog et al. 2014).

The $\mathrm{H}_{2} \mathrm{O}\left(2_{11}-2_{02}\right)$ line is well detected as well as the corresponding dust continuum. As shown by the images of the $\mathrm{H}_{2} \mathrm{O}$ and dust continuum (Fig. A.1), the source is partially resolved by the synthesised beam. The two component (east and 
west) structure is consistent with the $880 \mu \mathrm{m}$ image, and the western component is stronger than the eastern one. Both ratios of $S_{v}(\mathrm{ct})^{\mathrm{pk}} / S_{v}(\mathrm{ct})$ and $S_{\mathrm{H}_{2} \mathrm{O}}^{\mathrm{pk}} / S_{\mathrm{H}_{2} \mathrm{O}}$ are found to be 0.6 (uncertainty $<13 \%$ ). However, the eastern component is not detected in the $\mathrm{H}_{2} \mathrm{O}$ image.

The $\mathrm{H}_{2} \mathrm{O}\left(2_{11}-2_{02}\right)$ line can be fitted with a single Gaussian profile with a FWHM of $277 \pm 14 \mathrm{~km} \mathrm{~s}^{-1}$ (Fig. 2). However, the line has a steeper decline on the red side of the spectrum. The CO(4-3) observation gives a linewidth of $198 \pm 51 \mathrm{~km} \mathrm{~s}^{-1}$, which is $0.7 \pm 0.2$ times narrower than that of the $\mathrm{H}_{2} \mathrm{O}$ line. The velocity-integrated flux density of the $\mathrm{H}_{2} \mathrm{O}$ is larger than that of the $\mathrm{CO}(4-3)$ in this source with a ratio of $I_{\mathrm{H}_{2} \mathrm{O}} / I_{\mathrm{CO}}=1.1 \pm 0.3$.

\section{A.8. SDP11 at $z=1.786$}

The CO observation by Lupu et al. (2012) found $z=1.786$. The SMA $880 \mu \mathrm{m}$ image displays a typical strongly lensed morphology with two components, north and south, respectively. The size of the source is $\sim 2^{\prime \prime}$. The gravitational magnification estimated by $\mathrm{B} 13$ is $\mu=10.9 \pm 1.3$.

As shown in Fig. A.1, the source is partially resolved. The dust continuum image shows an extended structure along the north and south direction, with the brightest peak in the northern part. The noisy images are consistent with the SMA $880 \mu \mathrm{m}$ observation. The ratio of $S_{v}(\mathrm{ct})^{\mathrm{pk}} / S_{v}(\mathrm{ct})$ is $0.56 \pm 0.03$. The image shows two distinctive components in the north and south direction. This structure also agrees with the high resolution SMA $880 \mu \mathrm{m}$ image. Additionally, $S_{\mathrm{H}_{2} \mathrm{O}}^{\mathrm{pk}} / S_{\mathrm{H}_{2} \mathrm{O}}=0.4 \pm 0.2$ suggests that the $\mathrm{H}_{2} \mathrm{O}$ image is slightly resolved compared with the dust emission. This diffierence may come from their different spatial distribution.

Although the noise level of its spectrum is the highest among our sources because of the high frequency, namely $355 \mathrm{GHz}$, the $\mathrm{H}_{2} \mathrm{O}\left(2_{02}-1_{11}\right)$ line is marginally detected with $S / N=4.6$. The linewidth is $214 \pm 41 \mathrm{~km} \mathrm{~s}^{-1}$ (Fig. 2).

\section{A.9. $N C v 1.268$ at $z=3.675$}

The redshift of NCv1.268 was obtained by the CO observation of Krips et al. (in prep.). A typical strongly lensed morphology was found by the SMA $880 \mu \mathrm{m}$ observation (B13), with a strong arc-like component in the south direction. The structure has a size $\sim 2.5^{\prime \prime}$. The B13 lensing model estimates $\mu=13.0 \pm 1.5$.

The source is marginally resolved by the NOEMA synthesis beam (Fig. A.1). When comparing the flux ratios between the dust and $\mathrm{H}_{2} \mathrm{O}$ emission from the peak and the spatially integrated values, they give $S_{v}(\mathrm{ct})^{\mathrm{pk}} / S_{v}(\mathrm{ct})=0.66 \pm 0.01$ and $S_{\mathrm{H}_{2} \mathrm{O}}^{\mathrm{pk}} / S_{\mathrm{H}_{2} \mathrm{O}}=$ $0.6 \pm 0.1$. The values of dust emission and the $\mathrm{H}_{2} \mathrm{O}$ image are in good agreement.

NCv1.268 is the only source in which we have detected a double-peaked line profile from our new observations, with a slightly stronger blue velocity component (Fig. 2). The total linewidth is very large, $731 \pm 75 \mathrm{~km} \mathrm{~s}^{-1}$.

\section{A.10. NAv1.56 at $z=2.301$}

Harris et al. (2012) give a CO redshift of $z=2.3010$ for this source. The SMA $880 \mu \mathrm{m}$ dust continuum image shows a classic strongly lensed morphology with multiple images. It consists of an arc-like component in the western direction and a point-like component toward the east. They are separated $\sim 2^{\prime \prime}$. The lens model implies that the magnification factor $\mu=11.7 \pm 0.9$ (B13).
As shown in Fig. A.1, the source is marginally resolved, with an extended morphology in the eastern part. The structures displayed in the dust and $\mathrm{H}_{2} \mathrm{O}$ images are similar. The ratios $S_{v}(\mathrm{ct})^{\mathrm{pk}} / S_{v}(\mathrm{ct})=0.62 \pm 0.03$ and $S_{\mathrm{H}_{2} \mathrm{O}}^{\mathrm{pk}} / S_{\mathrm{H}_{2} \mathrm{O}}=0.7 \pm 0.2$ also suggest their similarity within the errors. Most of the fluxes are concentrated in the western part, which agrees with the SMA $880 \mu \mathrm{m}$ image.

The $\mathrm{H}_{2} \mathrm{O}\left(2_{11}-2_{02}\right)$ line can be fitted by a single Gaussian with a large linewidth equal to $593 \pm 56 \mathrm{~km} \mathrm{~s}^{-1}$. The $\mathrm{CO}(4-3)$ line observation by NOEMA (Oteo et al., in prep.) gives a linewidth of $621 \pm 47 \mathrm{~km} \mathrm{~s}^{-1}$. The linewidths of $\mathrm{CO}$ and $\mathrm{H}_{2} \mathrm{O}$ are in very good agreement. Our noisy detection of $\mathrm{CO}(5-4)$ at IRAM $30 \mathrm{~m}$ (Table 3 ) gives a ratio of $I_{\mathrm{H}_{2} \mathrm{O}} / I_{\mathrm{CO}}=0.8 \pm 0.3$.

\section{A.11. G09v1.124 at $z=2.410$}

The redshift of this source is measured by $\mathrm{CO}$ observation (Harris et al. 2012). This multiple source, with two main components, each with intrinsic $L_{\mathrm{IR}}>10^{13} L_{\odot}$, separated by $10^{\prime \prime}$ (Fig. A.1), was studied in detail by (Ivison et al. 2013, see also B13 and Oteo et al., in prep.). It is special in our sample since the two main sources are from two very different HyLIRGs rather than multiple images of a single source generated by strong gravitational lensing. The eastern component G09v1.124-W, which contains a powerful AGN (Oteo et al., in prep.), is unlensed and the western component G09v1.124-T is only weakly lensed with a magnification factor $\mu=1.5 \pm 0.2$. Thus, throughout the discussions, we treat G09v1.124-W and G09v1.124-T as two distinct sources (see Tables 2, 4, 5 and 6).

Probably because of this too small lensing magnification and the smaller values of each $\mu L_{\mathrm{IR}}$ (Table 2), we have only detected the dust continuum emission in this source. The $\mathrm{H}_{2} \mathrm{O}\left(2_{11}-2_{02}\right)$ line is undetected. The $2 \sigma$ upper limits of the velocity-integrated flux density of the $\mathrm{H}_{2} \mathrm{O}\left(2_{11}-2_{02}\right)$ line show that the values of $I_{\mathrm{H}_{2} \mathrm{O}}$ are more than three times smaller than in the other sources. As seen in Table 4 and Fig. 3, the ratio $L_{\mathrm{H}_{2} \mathrm{O}} / L_{\mathrm{IR}}$ is smaller than all our other sources for G09v1.124-W, probably because of its strong AGN. However, for G09v1.124-T this ratio, albeit small, might be comparable to that of G09v1.97. The dust continuum at $221 \mathrm{GHz}$ follows the same structure as the previously published observations (Fig. A.1 and Ivison et al. 2013). Both the eastern component (W) and the western one (T) are marginally resolved by the synthesised beam. The peak to total continuum flux ratios are $S_{v}(\mathrm{ct})^{\mathrm{pk}} / S_{v}(\mathrm{ct})=0.84 \pm 0.03$ and $0.83 \pm 0.04$, respectively.

\section{Appendix $\mathrm{B}: \mathrm{H}_{2} \mathrm{O}^{+}$detections in local ULIRGs}

The study using the Herschel SPIRE/FTS spectra of 167 local galaxies has revealed several emission and absorption lines of $\mathrm{H}_{2} \mathrm{O}^{+}$, which are ortho- $\mathrm{H}_{2} \mathrm{O}^{+}$lines $211-2_{02}(5 / 2-5 / 2)$, $2_{02}-1_{11(5 / 2-3 / 2)}, 1_{11}-0_{00(3 / 2-1 / 2)}, 1_{11}-0_{00(1 / 2-1 / 2)}$ (Y13, see also Table B.1). All $J \geq 2 \mathrm{H}_{2} \mathrm{O}^{+}$lines are in emission. Table B.1 gives values of the $\mathrm{H}_{2} \mathrm{O}^{+}$flux and luminosity for those among the Y13 sample where $\mathrm{H}_{2} \mathrm{O}^{+}$lines are (tentatively) detected with $S / N \gtrsim 2.5$. However, for the $\mathrm{H}_{2} \mathrm{O}^{+}\left(1_{11}-0_{00}\right)$ lines which connect the ground state, they are often found to be in emission in AGN-dominated sources while they are in absorption in star-forming-dominated ones. A full description of the dataset for this Herschel SPIRE/FTS survey will be given in Liu et al. in prep. At high-redshift prior to our study, the $J=2$ ortho$\mathrm{H}_{2} \mathrm{O}^{+}$doublet lines seem to have only been tentatively detected in two sources, SPT0346-52 (Weiß et al. 2013) and HFLS3 (Riechers et al. 2013). 
C. Yang et al.: $\mathrm{H}_{2} \mathrm{O}$ excitation in lensed $\mathrm{Hy} /$ ULIRGs at $z \sim 2-4$

Table B.1. Beam-matched $\mathrm{H}_{2} \mathrm{O}^{+}, \mathrm{H}_{2} \mathrm{O}$ line and infrared luminosities from local detections (Herschel SPIRE/FTS archive) and high-redshift Herschel lensed galaxies.

\begin{tabular}{|c|c|c|c|c|}
\hline Source & $\begin{array}{c}2_{02}-1_{11}(5 / 2-3 / 2) \\
10^{6} L_{\odot} \\
\end{array}$ & $\begin{array}{c}2_{11}-2_{02}(5 / 2-5 / 2) \\
10^{6} L_{\odot} \\
\end{array}$ & $\begin{array}{c}L_{\mathrm{IR}-\text { beam }} \\
10^{11} L_{\odot} \\
\end{array}$ & $\begin{array}{r}L_{\mathrm{H}_{2} \mathrm{O}} \\
10^{6} L_{\odot} \\
\end{array}$ \\
\hline \multicolumn{5}{|c|}{ Local ULIRGs } \\
\hline ESO320-G030 & $0.4 \pm 0.1$ & _- & 1.5 & 0.9 \\
\hline CGCG049-057 & $0.3 \pm 0.1$ & $0.7 \pm 0.2$ & 1.9 & 1.6 \\
\hline NGC 2623 & $0.5 \pm 0.2$ & $0.7 \pm 0.3$ & 3.5 & 2.0 \\
\hline Arp299-A & $0.8 \pm 0.2$ & $0.7 \pm 0.3$ & 5.4 & 1.3 \\
\hline Arp220 & $3.7 \pm 0.8$ & $3.5 \pm 0.9$ & 16.2 & 15.2 \\
\hline IRAS13120-5453 & $3.6 \pm 1.2$ & $2.4 \pm 1.0$ & 28.2 & 10.7 \\
\hline IRASF17207-0014 & $6.8 \pm 2.2$ & $6.3 \pm 2.2$ & 24.6 & 15.5 \\
\hline Mrk231 & $2.6 \pm 1.0$ & $3.0 \pm 1.5$ & 32.4 & 9.3 \\
\hline \multicolumn{5}{|c|}{ High-redshift lensed galaxies } \\
\hline NCv1.143 & $28.7 \pm 7.2$ & $28.6 \pm 7.2$ & 113.6 & 89.7 \\
\hline G09v1.97 & $35.8 \pm 9.0$ & - & 211.4 & 106.6 \\
\hline G15v2.779 & - & $55.8 \pm 15.9$ & 270.0 & 266.0 \\
\hline HFLS3 & - & $261.8 \pm 200.3$ & 364.0 & 737.8 \\
\hline
\end{tabular}

Notes. Luminosity of $\mathrm{H}_{2} \mathrm{O}^{+}$fine structure lines $2_{02}-1_{11(5 / 2-3 / 2)}$ (742.1 GHz), $2_{11}-2_{02}(5 / 2-5 / 2)(746.5 \mathrm{GHz})$, and $\mathrm{H}_{2} \mathrm{O}\left(2_{11}-2_{02}\right)$. As for the local sources the Herschel SPIRE/FTS beam is smaller than the entire source for some galaxies, here $L_{\mathrm{IR} \text {-beam }}$ represents the $L_{\mathrm{IR}}$ from the same beam area as the measured emission line (a full description will be given by Liu et al., in prep.). $L_{\mathrm{H}_{2} \mathrm{O}}$ is the luminosity of $\mathrm{H}_{2} \mathrm{O}\left(2_{11}-2_{02}\right)$. HFLS3 data are taken from Riechers et al. (2013). 